\section{orin}
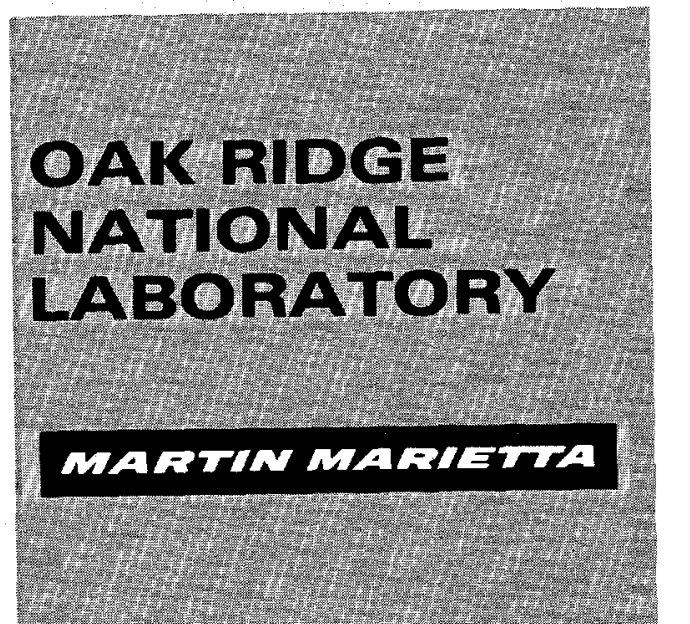

\section{in}

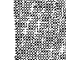
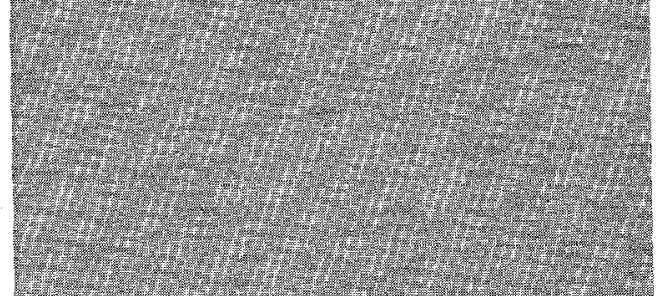

tre

res
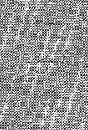

(1)
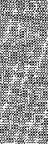

$4+2 x^{2}$

.

站:

(2)

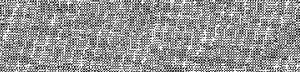

(2.

\section{MANAGED BY}

WARTIN MARIETA ENERGY SYSTEMS, INO.

FOR THE UMIED STATES

DEPARTMENT OF ENEROY

\section{SAMDIST: A Computer Code for Calculating Statistical Distributions for R-Matrix Resonance Parameters}

\author{
L. C. Leal \\ N. M. Larson
}



oRNL/TM-13092

Computational Physics and Engineering Division

\title{
SAMDIST: A COMPUTER CODE FOR CALCULATING STATISTICAL DISTRIBUTIONS FOR R-MATRIX RESONANCE PARAMETERS
}

\author{
L. C. Leal \\ N. M. Larson
}

Manuscript Completed: September 1995

Date Published: September 1995

Prepared by the

OAK RIDGE NATIONAL LABORATORY

managed by

LOCKHEED MARTIN ENERGY SYSTEMS, INC.

for the

U.S. DEPARTMENT OF ENERGY

under contract DE-AC05-84OR21400 

WTS OF

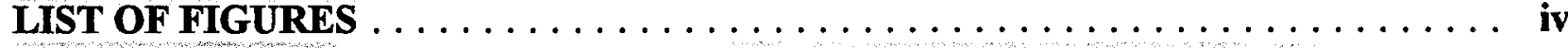
LIST OF TABLES $\ldots \ldots \ldots \ldots \ldots \ldots \ldots \ldots \ldots \ldots \ldots \ldots \ldots \ldots \ldots \ldots \ldots, \mathbf{v}$ ACKNOWLEDGMENTS $\ldots \ldots \ldots \ldots \ldots \ldots \ldots \ldots \ldots \ldots \ldots \ldots \ldots$ vii

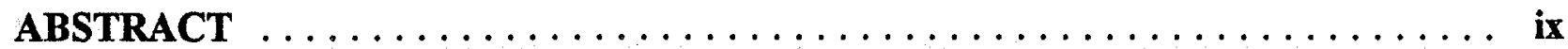

1. INTRODUCTION $\ldots \ldots \ldots \ldots \ldots \ldots \ldots \ldots \ldots \ldots \ldots \ldots \ldots \ldots$

2. BRIEF OVERVIEW OF THE THEORETICAL DISTRIBUTIONS OF THE RESONANCE PARAMETERS $\ldots \ldots \ldots \ldots \ldots \ldots \ldots \ldots \ldots 2$

2.1 LEVEL SPACING DISTRIBUTION LAW $\ldots \ldots \ldots \ldots \ldots \ldots \ldots \ldots 2$

2.2 RESONANCE WIDTH DISTRIBUTION LAW ............. 2

2.3 DYSON AND MEHTA LONG-RANGE CORRELATION OF $\Delta_{3}$ STATISTICS TEST $\ldots \ldots \ldots \ldots \ldots \ldots \ldots \ldots \ldots \ldots \ldots \ldots$

3. SAMPLING PROCEDURE $\ldots \ldots \ldots \ldots \ldots \ldots \ldots \ldots \ldots \ldots \ldots$ 3.1 FIRST AND SECOND MOMENTS, VARIANCE AND STANDARD DEVIATION $\ldots \ldots \ldots \ldots \ldots \ldots \ldots \ldots \ldots \ldots \ldots, 8$

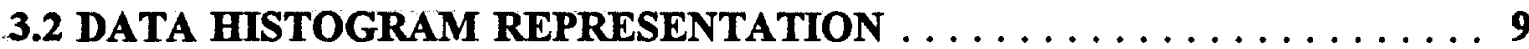

4. RUNNING SAMDIST $\ldots \ldots \ldots \ldots \ldots \ldots \ldots \ldots \ldots \ldots \ldots \ldots \ldots$

5. SAMDIST OUTPUT $\ldots \ldots \ldots \ldots \ldots \ldots \ldots \ldots \ldots \ldots \ldots \ldots$

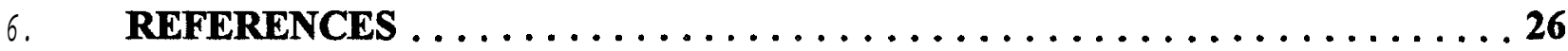

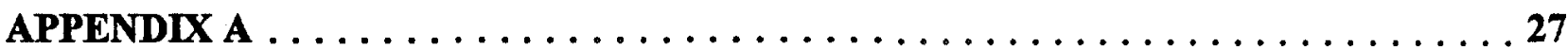

APPENDIX B $\ldots \ldots \ldots \ldots \ldots \ldots \ldots \ldots \ldots \ldots \ldots \ldots \ldots \ldots \ldots \ldots \ldots \ldots \ldots \ldots \ldots$ 


\section{LIST OF FIGURES}

Figure

$\underline{\text { Page }}$

1 Level spacing distribution for $\mathrm{J}=3$. Calculations (solid line) compared with

Wigner distribution (dashed line) $\ldots \ldots \ldots \ldots \ldots \ldots \ldots \ldots, \ldots, \ldots \ldots, 15$

2 Level spacing distribution for $\mathrm{J}=4$. Calculations (solid line) compared with

Wigner distribution (dashed line) $\ldots \ldots \ldots \ldots \ldots \ldots \ldots \ldots \ldots \ldots \ldots \ldots \ldots \ldots \ldots$

3 Reduced neutron-width distribution for $\mathrm{J}=3$. Calculations (solid line)

compared with Porter-Thomas distribution (dashed line) $\ldots \ldots \ldots, \ldots \ldots \ldots, 18$

4 Reduced neutron-width distribution for $\mathrm{J}=4$. Calculations (solid line)

compared with Porter-Thomas distribution (dashed line) . . . . . . . . . . . 19

$5 \quad$ Fission-width distribution for $\mathrm{J}=3$. Calculations (solid line) compared with

$\chi^{2}$ distribution with four degrees of freedom (dashed line) $\ldots \ldots \ldots, \ldots, \ldots, 20$

$6 \quad$ Fission-width distribution for $\mathrm{J}=4$. Calculations (solid line) compared with

$\chi^{2}$ distribution with four degrees of freedom (dashed line) $\ldots \ldots \ldots \ldots \ldots \ldots 21$

7 Cumulative number of energy levels vs energy for $\mathrm{J}=3 \ldots \ldots \ldots \ldots \ldots$

8 Cumulative number of energy levels vs energy for $J=4 \ldots \ldots \ldots \ldots \ldots, 25$ 


\section{LIST OF TABLES}

$\underline{\text { Table }}$

Page

$1 \quad$ Nearest-neighbor-spacing distribution for $\mathrm{J}=3 \ldots \ldots, \ldots \ldots \ldots \ldots \ldots$

2 Nearest-neighbor-spacing distribution for $\mathrm{J}=4 \ldots \ldots \ldots \ldots \ldots \ldots \ldots \ldots \ldots \ldots$

$3 \quad$ Reduced neutron-width distribution for $\mathrm{J}=3 \ldots \ldots \ldots \ldots \ldots \ldots \ldots \ldots \ldots$

$4 \quad$ Reduced neutron-width distribution for $J=4 \ldots \ldots \ldots \ldots \ldots \ldots \ldots \ldots \ldots \ldots$

$5 \quad$ Fission-width distribution with four degrees of freedom for $\mathrm{J}=3 \ldots \ldots \ldots \ldots$

6 Fission-width distribution with four degrees of freedom for $\mathrm{J}=4 \ldots \ldots \ldots \ldots 21$

$7 \quad$ The $\mathrm{A}$, results for $\mathrm{J}=3$ (only the first $30{ }^{235} \mathrm{U}$ s-wave resonances are shown) $\ldots \ldots 22$

$8 \quad$ A, results for $\mathrm{J}=4$ (only the first $30{ }^{233} \mathrm{U}$ s-wave resonances are shown) $\ldots \ldots \ldots 24$ 



\section{ACKNOWLEDGMENTS}

This work was sponsored by Defense Programs, U.S. Department of Energy (DOE), under contract DE-AC05-84OR21400 with Lockheed Martin Energy Systems, Inc. The authors are. particularly indebted to Col. James Felty, DOE-Washington, for his support. 
. 


\begin{abstract}
'The: SAMDIST computer code has been develoged to calculate distribution of resonance parameters of the Reich-Moore R-matrix type. The program assumes the parameters are in the format compatible with that of the multilevel R-matrix code SAMMY.

SAMDIST calculates the energy-level spacing distribution, the resonance width distribution, and the long-range correlation of the energy levels. Results of these calculations are presented in both graphic and tabular forms.
\end{abstract}





\section{INTRODUCTION}

The existence of statistical distributions for R-matrix resonance parameters has important implications for data analyses in both the resolved and the unresolved energy regions. In the resolved energy region, an evaluator may encounter diiculties in obtaining a set of resonance parameters that fit simultaneously various'sets of experimental data. The most common source of these difficulties is the broadening of the data due to finite experimental resolution; this broadening may preclude the identification of some smallresonance levels. In such a case, the known statistical distributions of the resonance parameters can be used to provide guidance for the location and the magnitudes of missing levels in the resonance set. In the unresolved energy region, the statistical distributions of the resonance parameters can be used to generate average cross sections.

The purpose of this work is to describe a tool, the code SAMDIST, which can be used in conjunction with a cross-section evaluation code such as SAMMY ${ }^{1}$ to verify the consistency of a resonance parameter set with the predicted theoretical statistical distribution.

The SAMDIST code has been designed for calculating distributions of resonance parameters of the Reich-Moore R-matrix type. The program accommodates resonance parameters in a format compatible with that of the SAMMY code. SAMDIST calculates distributions of the resonance parameters and compares them with theoretical predictions; results of those calculations are given in graphic and tabular forms. Average values and standard deviations are also given. A listing of the SAMDIST program is given in Appendix A.

The following tasks can be performed with the SAMDIST code:

1. Level spacing distributions may be determined according to the Wigner distribution law.

2. Distributions may be calculated for all widths, including neutron width, radiation width, and fission width (usually two channels in the Reich-Moore formalism). Values for each of these widths are distributed according to a $\chi^{2}$ distribution with the appropriate number of degrees of freedom.

3. Long-range correlations of the energies can be tested via the A, statistic test of Mehta-Dyson. 


\section{BRIEF OVERVIEW OF THE THEORETICAL DISTRIBUTIONS OF THE RESONANCE PARAMETERS}

\subsection{LEVEL SPACING DISTRIBUTION LAW}

The spacing between two consecutive resonance energies for the same total angular momentum and parity exhibits random behavior. For a set of $\boldsymbol{n}$ resonance energy levels, $\boldsymbol{E}, \boldsymbol{E}_{2}, \ldots$, $E_{n}$, where the level spacing between two consecutive energies, $E_{k}$ and $E_{k-1}$, is $D_{k}$, and the average level spacing is $\langle D\rangle$, the probability distribution function predicted by the Wigner law ${ }^{2}$ is

$$
\boldsymbol{p}(\boldsymbol{x}) d x=\frac{\pi x}{2} \exp \left(-\frac{\pi x^{2}}{4}\right) d x,
$$

where $\boldsymbol{x}=D_{k} /\langle D\rangle$, and $\langle D\rangle$ is the average level spacing. The Wigner probability distribution function has the following property:

$$
\int_{0}^{\infty} p(x) d x=\int_{0}^{\infty} x p(x) d x=1
$$

The second moment of the Wigner distribution is given by

$$
\overline{x^{2}}=\int_{0}^{\infty} x^{2} p(x) d x=\frac{4}{\pi} .
$$

Equation (1) was the first mathematical prediction of the level spacing distribution to provide excellent agreement with experimental results; it has triggered a series of investigations on the-subject of the statistical distribution of resonance parameters. Although other accurate level spacing distributions have been proposed, Wigner's law is the most widely used and is suitable for practical applications.

\subsection{RESONANCE WIDTH DISTRIBUTION LAW}

Systematic measurements of the resonance widths show strong fluctuations among resonances of the same angular momentum and parity. The definition of resonance width involves two other quantities, namely the reduced widths, $\boldsymbol{\gamma}_{\lambda_{c}}$, and the penetration factor, $P_{c}$, which are related according to the equation

$$
\Gamma_{\lambda}=\sum_{c}\left(2 P_{c}\right) \gamma_{\lambda c}^{2},
$$


where $\lambda$ refers to the energy levels in the compound nucleus and $\mathrm{c}$ refers to the particle channel. One should expect that the fluctuations are connected to either the reduced widths, $\boldsymbol{\gamma}_{\lambda c}$, or to the penetration factors, $P_{c}$. However, it is improbable that the fluctuations are due to the penetration factors since they are smooth functions of energy. Therefore, the-observed fluctuations are caused by the reduced widths, $\boldsymbol{\gamma}_{\lambda c}$; these, in turn are related to the projection of the eigenfunctions of the Hamiltonian of the compound nucleus on the nuclear surface. This projection involves an integration of many uncorrelated contributions, positive and negative, over the high-dimensional phase space of the compound nucleus. It then follows from the central limit theorem that the distributions of $\boldsymbol{\gamma}_{\lambda_{c}}^{2}$ have a Gaussian distribution with zero-mean. Therefore, the distribution function of the reduced widths can be written as

$$
P\left(\gamma_{\lambda c}\right) d \gamma_{\lambda c}=\frac{1}{\sqrt{\left.2 \pi<\gamma_{\lambda c}^{2}\right\rangle}} \exp \left(-\frac{{ }^{2} \gamma_{\lambda c}}{2<\gamma_{\lambda c}^{2}>}\right) d \gamma_{\lambda c},
$$

where $\left\langle\gamma_{\lambda_{c}}^{2}\right\rangle$ is the average value of $\boldsymbol{\gamma}_{\lambda c}^{2}$.

The probability distribution function of the resonance widths, $\Gamma_{\lambda}$, can be derived from Eq. (3) as follows: The statistical theorem states that ify is a variable that is the sum of squares of $\mathrm{v}$ normally distributed zero-mean independent variables, then y is distributed according to a $\chi^{2}$ distribution with v degrees. of freedom. Therefore, the distribution of $\Gamma_{\lambda}$ is

$$
p,(x) d x=\frac{v}{2 G(v / 2)}(v x / 2)^{\frac{v}{2}-1} \exp (-v x / 2) d x,
$$

wherex $=\Gamma_{\lambda} /\langle\Gamma\rangle, G(v / 2)$ is the mathematical gamma function, and $\langle\Gamma\rangle$ is the average value of the width taken over a given energy range. For $\mathrm{v}=1$, Eq. (6) is well known as the PorterThomas $^{3}$ distribution law of the neutron width. It is generally accepted that fission is a few-channel process, and that there are only a limited number of effectively open channels; 2 or 3 degrees of freedom $(v=2$ or $v=3)$ are usually assumed in the fission width distribution. In the neutron capture event, a large number of capture channels are opened; the gamma width distribution is represented by a $\chi^{2}$ distribution with a large number of degrees of freedom $(\mathrm{v}-\mathrm{w})$, which corresponds to a Diracdelta function centered at $\Gamma_{y}=\langle\Gamma\rangle$.

The $\chi^{2}$ distribution function has the following property:

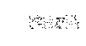

$$
\int_{0}^{\infty} p_{v}(x) d x=\int_{0}^{\infty} x p_{v}(x) d x=1
$$


4

The second moment of a $\boldsymbol{\chi}^{2}$ distribution with $\mathrm{v}$ degrees of freedom is given as

$$
\overline{x^{2}}=\int_{0}^{\infty} x^{2} p_{v}(x) d x=\frac{2}{v}+1
$$

\subsection{DYSON AND MEHTA LONG-RANGE CORRELATION OF $\Delta_{3}$ STATISTICS TEST}

Another useful tool for evaluating nuclear data is the $\Delta_{\mathbf{3}}$ statistics test introduced by Dyson and Mehta. ${ }^{4}$ The $\Delta_{3}$ test provides a measure of the mean-square deviation between the number of observed energy levels in the energy interval $E_{i}$ to $E_{f}$ and the best fit to the straight line, as a function of energy, given as $\boldsymbol{a} \boldsymbol{E}+\boldsymbol{b}$. Strictly speaking, the definition is

$$
\left.\Delta_{3}=\underset{(a, b)}{\operatorname{Min}} \mid \frac{1}{2 L} \int_{E_{i}}^{E_{f}}(N(E)-a E-b)^{2} d E\right],
$$

where $N(E)$ is the corresponding cumulative number of energy levels as a function of energy.

The Dyson and Mehta $\Delta_{\mathbf{3}}$ test predicts that the theoretical average value $<\Delta_{\mathbf{3}}>$ is given as

$$
<\Delta_{3}>=\frac{1}{\pi^{2}}[\ln (n)-0.06871
$$

with variance $V_{\Delta_{3}}=1.169 / \pi^{4}$. Here $n$ is the number of energy levels observed in the interval $E_{i}$ to $E_{f}$

For practical applications, the coefficients $\boldsymbol{a}$ and $\boldsymbol{b}$ in Eq. (9) are determined according to the following conditions:

$$
\frac{\partial \Delta_{3}}{\partial a}=0
$$

and

$$
\frac{\partial \Delta_{3}}{\partial b}=0
$$


These conditions lead to the following equations:

$$
\boldsymbol{a} \int_{E_{i}}^{E_{f}} E^{2} d E+\boldsymbol{b} \int_{E_{i}}^{E f} E d E=\int_{E_{i}}^{E_{f}} N(E) d E
$$

and

$$
a \int_{E_{i}}^{E_{f}} E d E+b \int_{E_{i}}^{E_{f}} d E=\int_{E_{i}}^{E_{f}} N(E) d E
$$

The toolıowing identtites will be used in evaluating $a$ and $b$ :

$$
\begin{gathered}
\int_{E_{i}}^{E_{f}} d E=E_{f}-E_{i}, \\
{ }_{E_{z}}^{E_{f}} E d E=\left(E_{f}^{2}-E_{i}^{2}\right) / 2,
\end{gathered}
$$

and

$$
{ }_{E_{f}}^{E_{f}} E^{2} d E=\left(E_{f}^{3}-E_{i}^{3}\right) / 3
$$

If the energy levels in the range $E_{i}$ to $E_{f}$ are numbered from $l=-\boldsymbol{L}$ to $\boldsymbol{l}=+\boldsymbol{L}$, then the following relations also hold:

$$
\int_{E_{l}}^{E_{f}} N(E) d E=\sum_{l=-L}^{+L} \int_{E_{l}}^{E_{l+1}} l d E=\sum_{l=-L}^{+L} l\left(E_{l+1}-E_{l}\right)
$$




$$
\int_{E_{t}}^{E_{f}} N(E) E d E=\sum_{l=-L}^{+L} \int_{E_{l}}^{E_{l+1}} l E d E=\sum_{l=-L}^{+L} l\left(E_{l+1}^{2}-E_{l}^{2}\right) / 2,
$$

and

$$
\underset{E_{i}}{E_{f}} N^{2}(E) E d E=\sum_{l=-L}^{+L} l^{2}\left(E_{l+1}-E_{l}\right)
$$

The system of Eqs. (13) and (14) can be written as

$$
\alpha_{1} a+\beta_{1} b=\gamma_{1}
$$

and

$$
\alpha_{2} a+\beta_{2} b=\gamma_{2},
$$

in which the Greek symbols are defined as

$$
\begin{gathered}
\int_{E_{i}}^{E_{f}} E d E=\left(E_{f}^{2}-E_{i}^{2}\right) / 2, \text { and } \\
\alpha_{2}=\beta_{1}=\left(E_{f}^{2}-E_{i}^{2}\right) / 2, \\
\beta_{2}=E_{f}-E_{i}, \\
\gamma_{1}=\sum_{l} l\left(E_{l+1}^{2}-E_{l}^{2}\right) / 2,
\end{gathered}
$$

and 


$$
\gamma_{n}=\sum_{i} l\left(E_{l+1}-E_{l}\right)
$$

The solution for $a$ and $b$ is then

$$
a=\frac{\gamma_{1}-\gamma_{2} \beta_{1} / \beta_{2}}{\alpha_{1}-\alpha_{2} \beta_{1} / \beta_{2}}
$$

and

$$
b \quad \frac{\gamma_{2}}{\beta_{2}}-\frac{\alpha_{2}}{\beta_{2}} \frac{\gamma_{1}-\gamma_{2} \beta_{1} / \beta_{2}}{\alpha_{1}-\alpha_{2} \beta_{1} / \beta_{2}}
$$

Substituting these definitions into Eq. (9) leads to the expression for the A, test:

$$
\Delta_{3}=\frac{1}{E_{f}-E_{i}}\left\{\int_{E_{i}}^{E_{f}} N^{2}(E) d E-\gamma_{1} a-\gamma_{2} b\right\}
$$

or

$$
\Delta_{3}=\frac{1}{E_{f}-E_{i}}\left\{\sum_{-L}^{+L} l^{2}\left(E_{l+1}-E_{l}\right)-\gamma_{1} a-\gamma_{2} b\right\}
$$

where $\boldsymbol{a}$ and $\boldsymbol{b}$ are given by Eqs. (28) and (29), and $\boldsymbol{\gamma}_{\mathbf{1}}$ and $\boldsymbol{\gamma}_{\mathbf{2}}$ by Eqs. (26) and (27). 


\section{SAMPLING PROCEDURE}

\subsection{FIRST AND SECOND MOMENTS, VARIANCE AND STANDARD DEVIATION}

The statistical sampling of the experimental data, such as the energy level spacing, the resonance width, etc., are carried out following the usual procedure applied in statistics. For a number $\boldsymbol{n}$ of random variables $\left(\boldsymbol{x}_{1}, \boldsymbol{x}_{2}, \ldots, \boldsymbol{x}_{n}\right)$ selected according to a probability distribution function, $\boldsymbol{f}(\boldsymbol{x})$, the estimation of the first moment, $\bar{x}$, also referred to as the mean, is given by

$$
\bar{x}=\frac{1}{n} \sum_{i=1}^{n} x_{i} .
$$

Similarly, the second moment is given by

$$
\overline{x^{2}}=\frac{1}{n} \sum_{i=1}^{n} x_{i}^{2} .
$$

The dispersion of the $\boldsymbol{x}_{\boldsymbol{i}}$ with respect to $\overline{\boldsymbol{x}}$ is defined as

$$
\sigma_{x_{i}}^{2}=\frac{1}{n-1} \sum_{i=1}^{n}\left(x_{i}-\bar{x}\right)^{2} .
$$

The variance of $\overline{\boldsymbol{x}}$ is given by

$$
\sigma_{\bar{x}}^{2}=\frac{1}{n} \sigma_{x_{i}}^{2}
$$

or

$$
\sigma_{\bar{x}}^{2}=\frac{1}{n(n-1)} \sum_{i=1}^{n}\left(x_{i}-\bar{x}\right)^{2},
$$

whereas the standard deviation, $\boldsymbol{s}$, is given by

$$
s=\sqrt{\sigma_{\bar{x}}^{2}} .
$$




\subsection{DATA HISTOGRAM REPRESENTATION}

The histogram distribution of the $n$ samples are obtained according to the following steps: ${ }^{5}$

1. The set of random variables $\left(x_{1}, x_{2}, \ldots, x_{n}\right)$ are ordered such that $x_{i}<x_{i+1}$.

2. For a user-defined bin width, $\boldsymbol{\delta} \boldsymbol{x}$, the number of intervals, $\boldsymbol{n i}$, is determined as

$$
n i=\frac{x_{n}}{\delta x} .
$$

3. The random variables $\left(\mathrm{x}_{1}, \boldsymbol{x}_{2}, \ldots, \boldsymbol{x}_{\boldsymbol{n}}\right)$ are sampled to determine the frequency in which $\boldsymbol{x}_{\boldsymbol{i}}$, for $\boldsymbol{i}=1, \ldots, \mathrm{n}$, falls in the interval between $(\boldsymbol{k}-1) \delta x$ and $k \delta x$, where $\mathrm{k}=1, \ldots, \boldsymbol{n i}$.

4. To calculate the probability $\boldsymbol{p}_{\boldsymbol{k}}$ of finding $\boldsymbol{x} \in\left(\boldsymbol{x}_{1}, \boldsymbol{x}_{2}, \dot{j}_{2}, \boldsymbol{x}_{\boldsymbol{n}}\right)$ in the $\boldsymbol{k}^{\text {th }}$ interval between $(k-1) \delta x$ and $k \delta x$, and the corresponding variance $\sigma_{k}^{2}$, and consequently the standard deviation $\boldsymbol{s}$, we note that each event in the $\boldsymbol{k}^{\text {th }}$ interval adds to a success, such as

$$
\xi_{\mathrm{ik}}= \begin{cases}1 & \text { event in the } k^{\text {th }} \text { interval }(i \in k) \\ 0 & \text { otherwise }(i \notin k)\end{cases}
$$

Therefore; the probability, $\boldsymbol{p}_{\boldsymbol{k}}$, is

$$
p_{k}=p((k-1) \delta x<x<k \delta x)=\frac{1}{n} \sum_{i=1}^{n} \xi_{i k},
$$

or

$$
p_{k}=\frac{k i}{n}
$$

where ki is the number of samples falling into the $\boldsymbol{k}^{\text {th }}$ interval.

The variance $\sigma_{k}^{2}$ is given by

$$
\sigma_{k}^{2}=\frac{1}{n(n-1)} \sum_{i=1}^{n}\left(\xi_{i k}-p_{k}\right)^{2}
$$

or 


$$
\sigma_{k}^{2}=\frac{1}{(n-1)} p_{k}\left(1-p_{k}\right)
$$

and the standard deviation, $\boldsymbol{s}$, is given as

$$
s=\sqrt{\frac{1}{(n-1)} p_{k}\left(1-p_{k}\right)}
$$




\section{RUNNING SAMDIST}

The SAMDIST program is written in FORTRAN77 on a RISC-6000 UNIX-based system. The input to SAMDIST is constructed by answering various prompts that ask for the type of the distribution, the name of the resonance parameters in the SAMMY format, the energy range in which the calculations are to be performed, etc. Two output files are produced as the result of a SAMDIST run: one of them is in ASCII format, named samdist.avg, while the other is in the FORODF format, ${ }^{6}$ named samdist.odf, which, in turn, can be displayed in graphic form. To illustrate the procedure to execute the SAMDIST program, the ${ }^{235} \mathrm{U}$ s-wave resonance parameters' are used. These represent the cross sections in the energy range from 0 to $500 \mathrm{eV}$ and are stored in the file 0to500.par. Two resonance spin groups are in the resonance parameter sets; these groups are specified by the numbers in the last columns of the file in the SAMMY format (for which a listing is displayed in Appendix B). In the following examples, the resonance parameter distributions are taken for the entire energy range from 0 to $500 \mathrm{eV}$. To distinguish program prompts from reply, the prompts'are given in boldface letters.

\section{a. Level-spacing distribution for spin group 1}

samdist

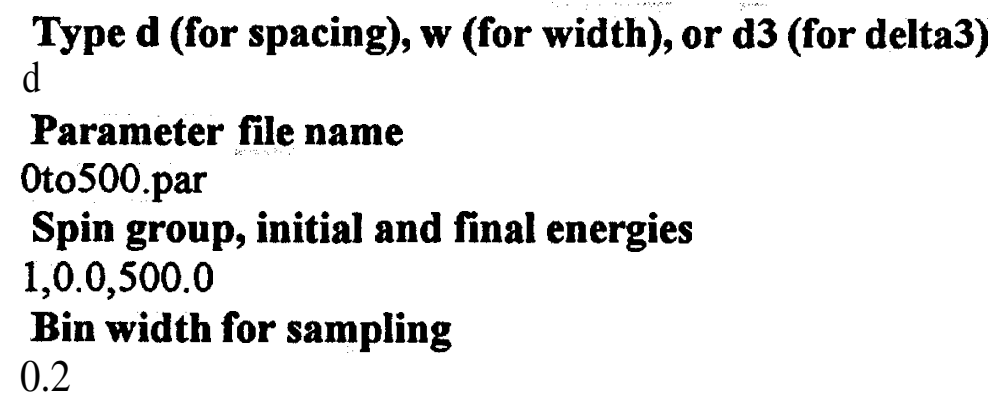

b. Level-spacing distribution for spin group 2

samdist

Type d (for spacing), w (for width), or d3 (for delta3)

d

Parameter file name

Oto500.par

Spin group, initial and final energies

$2,0.0,500.0$

Bin width for sampling

0.2 
c. Reduced neutron-width distribution for spin group 1

samdist

Type d (for spacing), w (for width), or d3 (for delta3)

W

Parameter file name

Oto500.par

Particle channel

neutron

Spin group, initial and final energies

$1,0.0,500.0$

Bin width for sampling

1.0

Degrees of freedom

1

d. Reduced neutron-width distribution for spin group 2

samdist

Type d (for spacing), w (for width), or d3 (for delta3)

W

Parameter file name

Oto500.par

Particle channel

neutron

Spin group, initial and final energies

2,0.0,500.0

Bin width for sampling

1.0

Degrees of freedom

1

e. Fission-width distribution for spin_group 1

samdist

Type d (for spacing), w (for width), or d3 (for delta3)

พ

Parameter file name

Oto500.par

Particle channel

fission

Spin group, initial and final energies

$1,0.0,500.0$ 
Bin width for sampling

1.0

Degrees of freedom

4

f. Fission-width distribution for spin group 2

samdist

Type d (for spacing), w (for width), or d3 (for delta3)

w

Parameter file name

Oto500.par

Particle channel

fission

Spin group, initial and final energies

$2,0.0,500.0$

Bin width for sampling

1.0

Degrees of freedom

4

g. $\Delta_{3}$ statistic test for spin group 1

samdist

Type d (for spacing), w (for width), or d3 (for delta3)

d3

Parameter file name

Oto500.par

Spin group, initial and final energies

$1,0.0,500.0$

h. $\underline{\Delta}_{3}$ statistic test for spin group 2

samdist

Type d (for spacing), w (for width), or d3 (for delta3)

d3

Parameter file name

Oto500.par

Spin group, initial and final energies

$2,0.0,500.0$ 


\section{SAMDIST OUTPUT}

Two output files, named samdist.avg and samdist.odf, are generated by a SAMDIST run. The samdist.avg output is in the BCD format, whereas the samdist.odf file is the graphic form of the statistical distribution, both of which were originated with the FORODF program. ${ }^{6}$ Description of the FORODF program can be found in ref. 6 . However, for completeness the FORODF statements used to generate the graphics shown here will be presented. The ASCII output contains average values calculated over the statistical distribution of the resonance parameters along with the standard deviations. The results of the calculations for the theoretical prediction are also provided. In addition to the average values and the standard deviations, the sampling distribution of the sampled variables is also given. It is the sampling distribution that is given in graphical form in the samdist.odf file. To illustrate the results of a SAMDIST calculation, the output obtained for each of the inputs described in the previous section (inputs a to $\mathrm{f}$ ) will be shown here. Recall that the data are ${ }^{235} \mathrm{U}$ s-wave resonance parameters of a SAMMY evaluation covering the energy range 0 to $500 \mathrm{eV}$.

\section{a. Level-spacing distribution for spin group 1}

The output created in this run is shown in Table 1, with the corresponding graphic output in Fig. 1. The FORODF sequence of statements used for generating the plot given in Fig. 1 is the following:

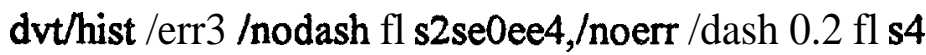

A complete explanation of the previous command is given in the FORODF manual. However, a brief description of each switch used in this command is as follows:

dvt is used to obtain the plot in the screen. It varies according to the hind of graphic device being used;

hist indicates to FORODF that the data will displayed in the form of histogram;

/err3 indicates that the standard deviations of the sampled variables, given by the vertical bars in the pictures, are in the position 3 in the FORODF file;

f1s2se0ee 4 indicates that the $x$ variable is stored in the position 1 and the theoretical distribution of $\mathrm{x}, \mathrm{p}(\mathrm{x})$ is in the position 2 ; se0ee4 indicates that $\mathrm{x}$ will span from 0 to 4 ;

/noerr indicates to turn off the /err3 switch;

/dash 0.2 indicates that the line will be dashed for differentiation purposes. The user may need to trigger this switch off for the next plot;

fls 4 indicates that the $\mathrm{x}$ variable is stored in the -position 1 and the experimental results is in the position 4 . 
Table 1. Nearest-neighbor-spacing distribution for $\mathrm{J}=3$

\begin{tabular}{|c|c|c|c|c|c|}
\hline $\begin{array}{l}d\rangle=1.406 \\
\text { number of leve } \\
\text { no. of levels } \\
\begin{array}{rlll}11 & 26 & 44 \\
4 & 7 & 4\end{array}\end{array}$ & $\begin{array}{l}63 E+ \\
\text { els' } \\
\text { in } \\
54 \\
41\end{array}$ & $\begin{array}{l}+00 \text { std }=3.8 \\
=35^{\circ} 5 \\
\text { each interv } \\
60 \quad 56 \quad 34\end{array}$ & 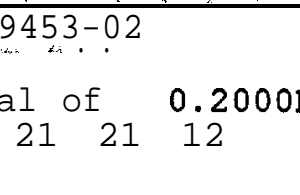 & $8+\infty$ & \\
\hline $\begin{array}{l}\text { Sampling } \\
0.0000 \mathrm{E}+00 \\
0.2000 \mathrm{E}+00 \\
0.4000 \mathrm{E}+00 \\
0.6000 \mathrm{E}+00 \\
0.8000 \mathrm{E}+00 \\
0.1000 \mathrm{E}+01 \\
0.1200 \mathrm{E}+01 \\
0.1400 \mathrm{E}+01 \\
0.1600 \mathrm{E}+01 \\
0.1800 \mathrm{E}+01 \\
0.2000 \mathrm{E}+01 \\
0.2200 \mathrm{E}+01 \\
0.2400 \mathrm{E}+01\end{array}$ & $\begin{array}{l}\text { Int } \\
= \\
= \\
- \\
= \\
= \\
= \\
= \\
= \\
= \\
=\end{array}$ & $\begin{array}{l}\text { erval } \\
0.2000 \mathrm{E}+00 \\
0.4000 \mathrm{E}+00 \\
0.6000 \mathrm{E}+00 \\
0.8000 \mathrm{E}+00 \\
0.1000 \mathrm{E}+01 \\
0.1200 \mathrm{E}+01 \\
0.1400 \mathrm{E}+01 \\
0.1600 \mathrm{E}+01 \\
0.1800 \mathrm{E}+01 \\
0.2000 \mathrm{E}+01 \\
0.2200 \mathrm{E}+01 \\
0.2400 \mathrm{E}+01 \\
0.2600 \mathrm{E}+01\end{array}$ & $\begin{array}{l}\text { Calculated } \\
0.3099 \mathrm{E}-01 \\
0-73243-01 \\
0.1239 \mathrm{E}+00 \\
0.1521 \mathrm{E}+00 \\
0.1690 \mathrm{E}+00 \\
0.1577 \mathrm{E}+00 \\
0.9577 \mathrm{E}-01 \\
0.59153-01 \\
0.59153-01 \\
0.33803-01 \\
0.1127 \mathrm{E}-01 \\
0.19723-01 \\
0.1127 \mathrm{E}-01 .\end{array}$ & $\begin{array}{l}\text { sdt } \\
0.9210 \mathrm{E}-02 \\
0.13853-01 \\
0.1751 \mathrm{E}-01 \\
0.1909 \mathrm{E}-01 \\
0.19923-01 \\
0.1937 \mathrm{E}-01 \\
0.15643-01 \\
0.1254 \mathrm{E}-01 \\
0.1254 \mathrm{E}-01 \\
0.96053-02 \\
0.5610 \mathrm{E}-02 \\
0.73893-02 \\
0.5610 \mathrm{E}-02\end{array}$ & $\begin{array}{l}\text { Theory } \\
0.3093 \mathrm{E}-01 \\
0.8716 \mathrm{E}-01 \\
0.1282 \mathrm{E}+00 \\
0.1488 \mathrm{E}+00 \\
0.1490 \mathrm{E}+00 \\
0.1332 \mathrm{E}+00 \\
0.1082 \mathrm{E}+00 \\
0.8061 \mathrm{E}-01 \\
0.554-13-01 \\
0.3528 \mathrm{E}-01 \\
0.2087 \mathrm{E}-01 \\
0.1149 \mathrm{E}-01 \\
0.5901 \mathrm{E}-02\end{array}$ \\
\hline
\end{tabular}

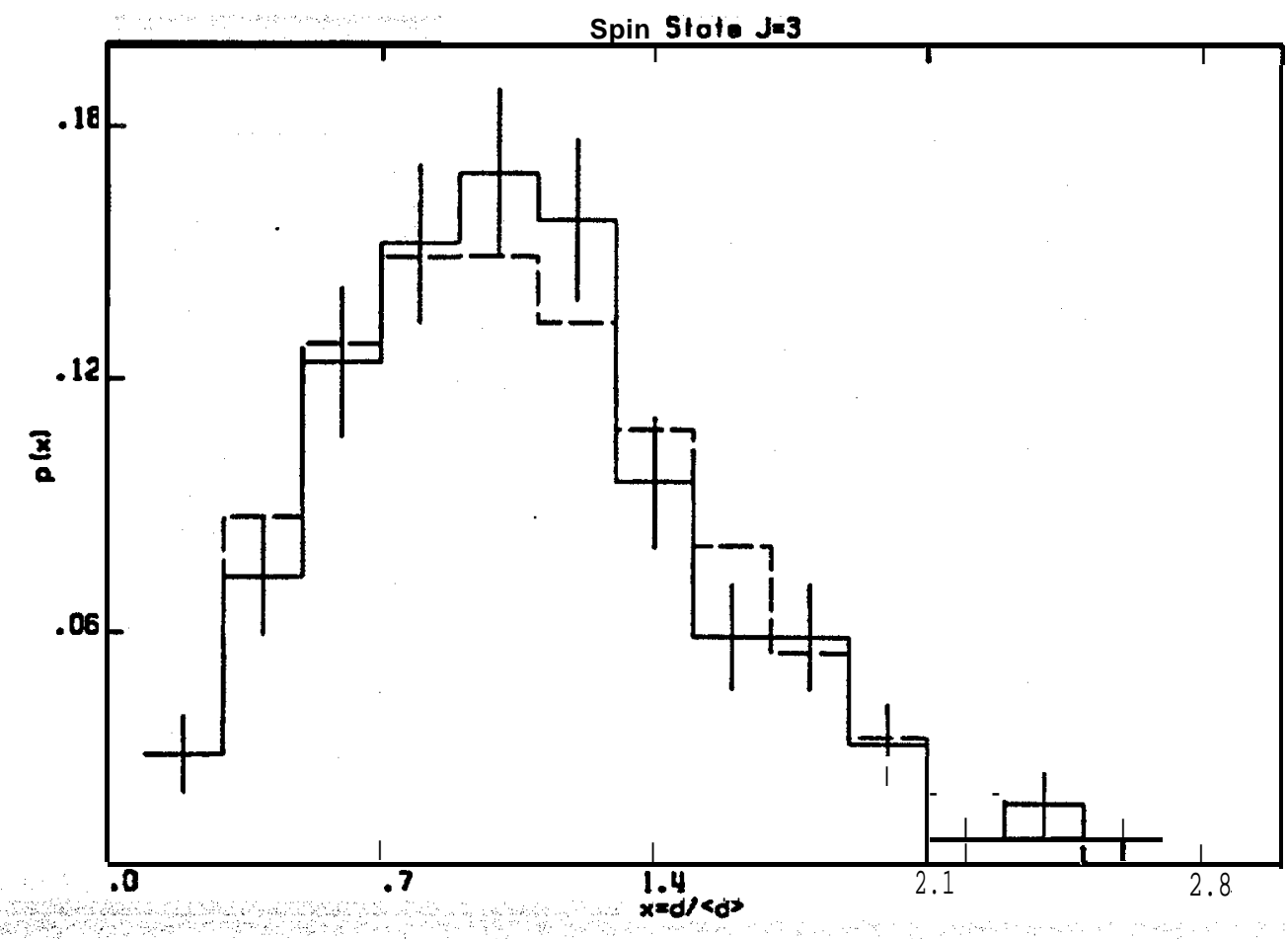

Fig. 1. Level spacing distribution for $\mathrm{J}=3$. Calculations (solid line) compared with Wigner distribution (dashed line). 
The FORODF switch for plotting the other results is very similar to the one just described, and, therefore, it will not be described. For users who do not have FORODF, it will be worthwhile to use the ASCII results given in the samdist.avg and construct the graphic output using any available plotting capability,

\section{b. Level-spacing distribution for spin group 2}

The output created in this run is shown in Table 2. The corresponding graphic output is given in Fig. 2. The FORODF sequence of statements used for generating the plot given in Fig. 2 is the following:

$\mathrm{dvt} /$ hist /err3 /nodash fl s2se0ee4,/noerr /dash $0.2 \mathrm{fl} \mathrm{s} 4$

Table 2. Nearest-neighbor-spacing distribution for $\mathrm{J}=4$

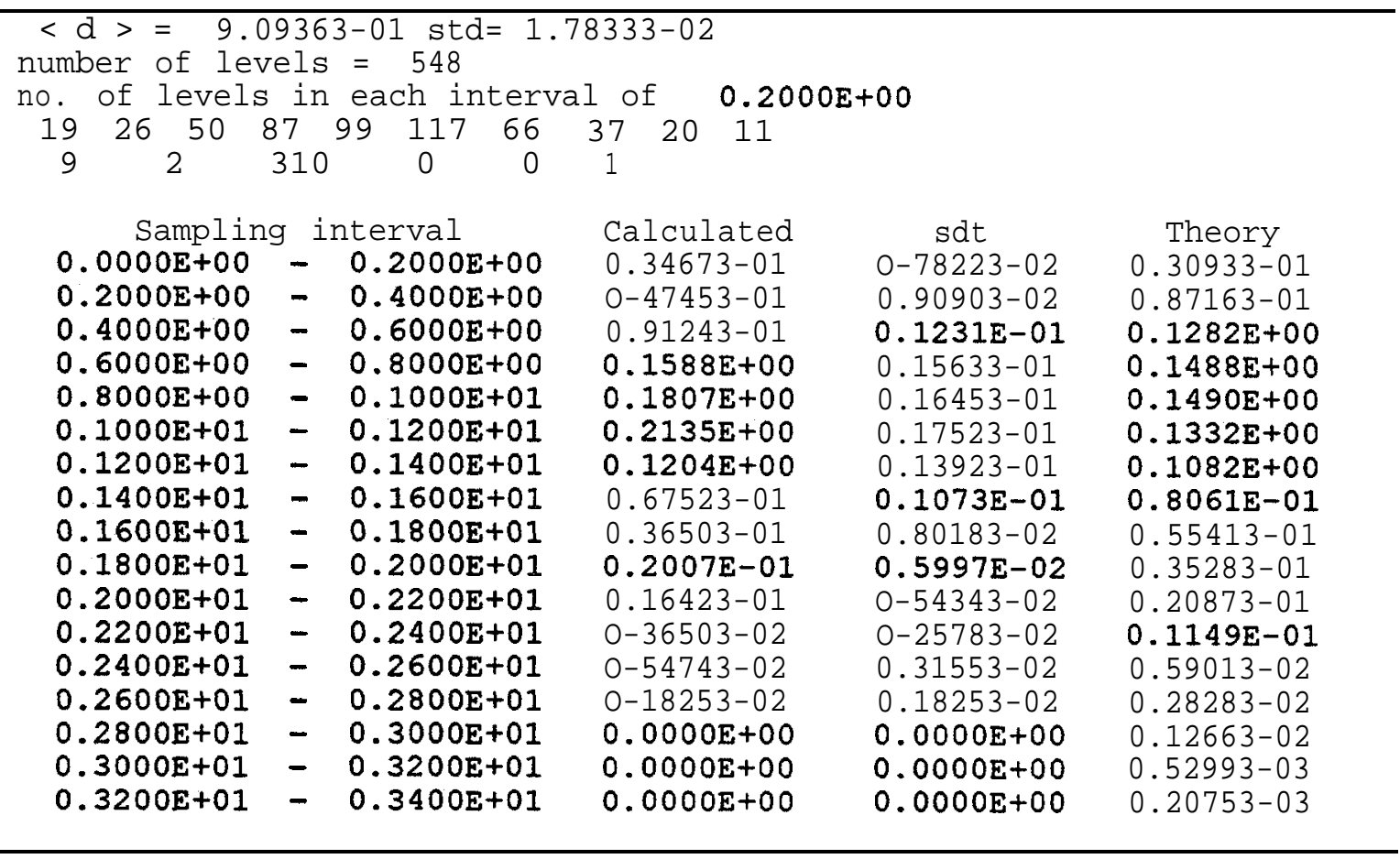




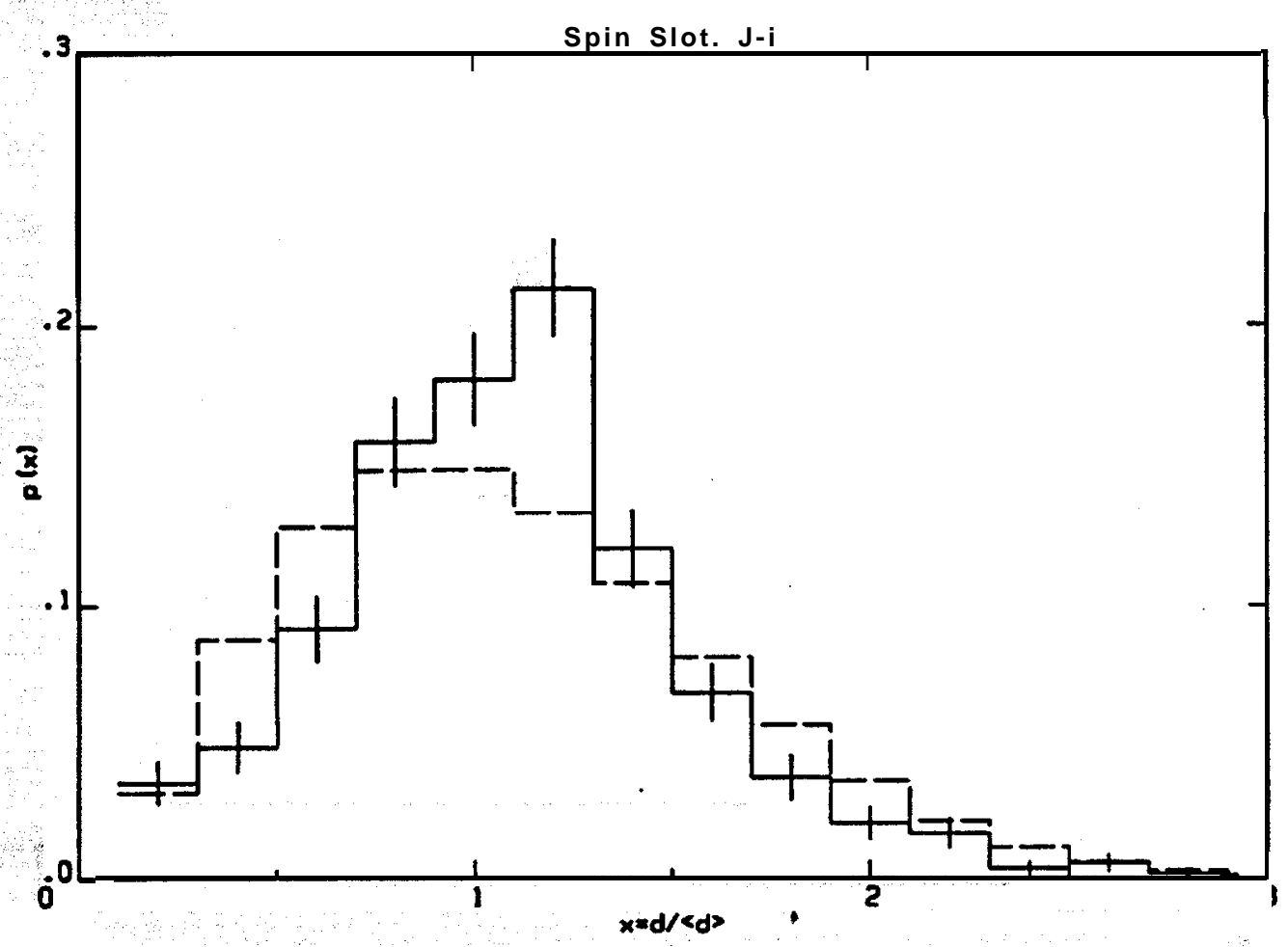

Fig. 2. Level spacing distribution for $J=4$. Calculations (solid line) compared with Wigner distribution (dashed line).

c. Reduced neutron-width distribution for spin group 1

The output created in this run is shown in Table 3. The corresponding graphic output is given in Fig. 3. The FORODF sequence of statements used for generating the plot given in Fig. 3 is the following:

dvt /hist /err3 /nodash f1 s2se0ee8,/noerr /dash 0.2 fls4 
Table 3. Reduced neutron-width distribution for $\mathrm{J}=3$

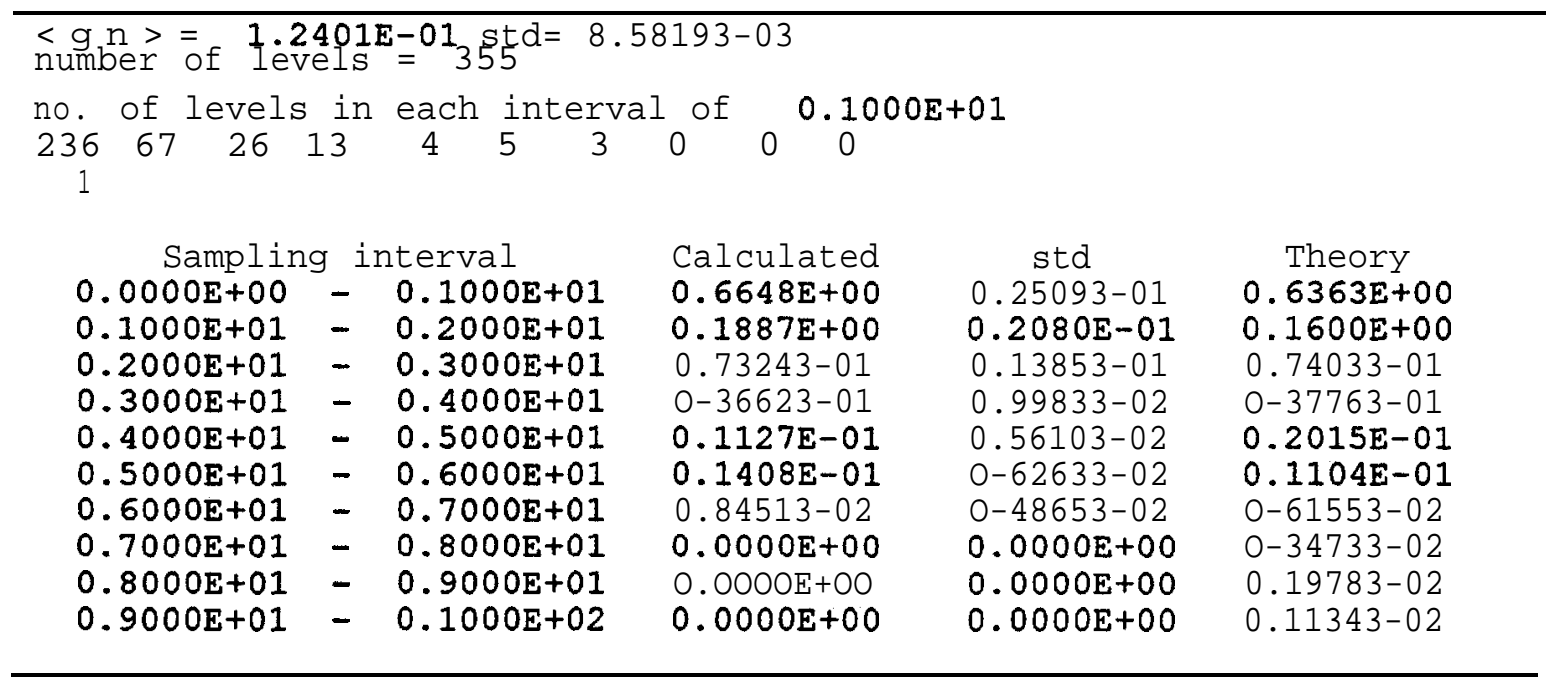

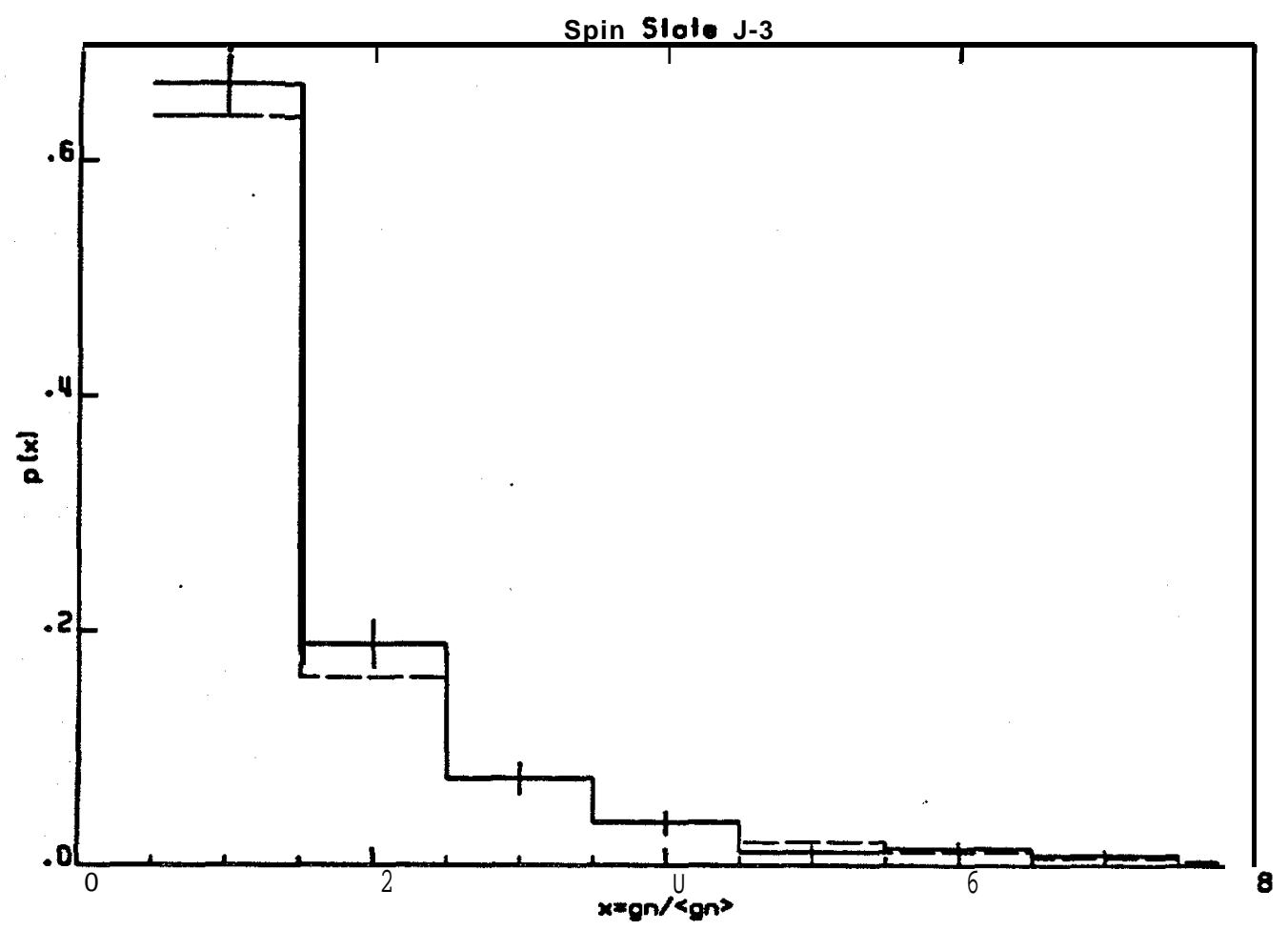

Fig. 3. Reduced neutron-width distribution for $\mathrm{J}=3$. Calculations (solid line) compared with Porter-Thomas distribution (dashed line). 


\section{d. Reduced neutron-width distribution for spin group 2}

The output created in this run is shown in Table 4. The corresponding graphic output is given in Fig. 4. The FORODF sequence of statements used for generating the plot given in Fig. 4 is the following:

$\mathrm{dvt} /$ hist /err3 /nodash f1s2se0ee8,/noerr /dash 0.2 fls4

Table 4. Reduced neutron-width distribution for $\mathrm{J}=4$

\begin{tabular}{|c|c|c|c|c|}
\hline 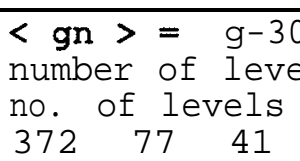 & $\begin{array}{l}243-02 \text { std }=5.6 \\
\text { is }=549 \\
\text { in each interva } \\
33 \quad 14 \quad 63\end{array}$ & 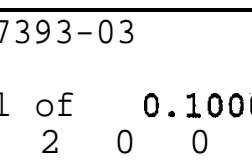 & $\begin{array}{l}E+01 \\
1\end{array}$ & \\
\hline $\begin{array}{l}\text { Sampling } \\
0.0000 \mathrm{E}+00 \\
0.1000 \mathrm{E}+01 \\
0.2000 \mathrm{E}+01 \\
0.3000 \mathrm{E}+01 \\
0.4000 \mathrm{E}+01 \\
0.5000 \mathrm{E}+01 \\
0.6000 \mathrm{E}+01 \\
0.7000 \mathrm{E}+01 \\
0.8000 \mathrm{E}+01 \\
0.9000 \mathrm{E}+01\end{array}$ & $\begin{array}{ll} & \text { interval } \\
- & 0.1000 \mathrm{E}+01 \\
- & 0.2000 \mathrm{E}+01 \\
- & 0.3000 \mathrm{E}+01 \\
= & 0.4000 \mathrm{E}+01 \\
- & 0.5000 \mathrm{E}+01 \\
= & 0.6000 \mathrm{E}+01 \\
- & 0.7000 \mathrm{E}+01 \\
= & 0.8000 \mathrm{E}+01 \\
- & 0.9000 \mathrm{E}+01 \\
- & 0.1000 \mathrm{E}+02\end{array}$ & $\begin{array}{l}\text { Calculated } \\
0.6776 \mathrm{E}+00 \\
0.1403 \mathrm{E}+00 \\
0.7468 \mathrm{E}-01 \\
0.6011 \mathrm{E}-01 \\
0.2550 \mathrm{E}-01 \\
0.11093 \mathrm{E}-01 \\
0.5464 \mathrm{E}-02 \\
0.3643 \mathrm{E}-02 \\
0.0000 \mathrm{E}+00 \\
0.0000 \mathrm{E}+00\end{array}$ & $\begin{array}{c}\text { std } \\
0.19973-01 \\
0.14833-01 \\
0.1123 \mathrm{E}-01 \\
0.1015 \mathrm{E}-01 \\
0-67343102 \\
0.4441 \mathrm{E}-02 \\
0.3149 \mathrm{E}-02 \\
0.2574 \mathrm{E}-02 \\
0.0000 \mathrm{E}+00 \\
0.0000 \mathrm{E}+00\end{array}$ & $\begin{array}{l}\text { Theory } \\
0.6363 \mathrm{E}+00 \\
0.1600 \mathrm{E}+00 \\
0.7403 \mathrm{E}-01 \\
0-37763-01 \\
0.2015 \mathrm{E}-01 \\
0.1104 \mathrm{E}-01 \\
0.61553-02 \\
0-34733-02 \\
0.1978 \mathrm{E}-02 \\
0.11343-02\end{array}$ \\
\hline
\end{tabular}

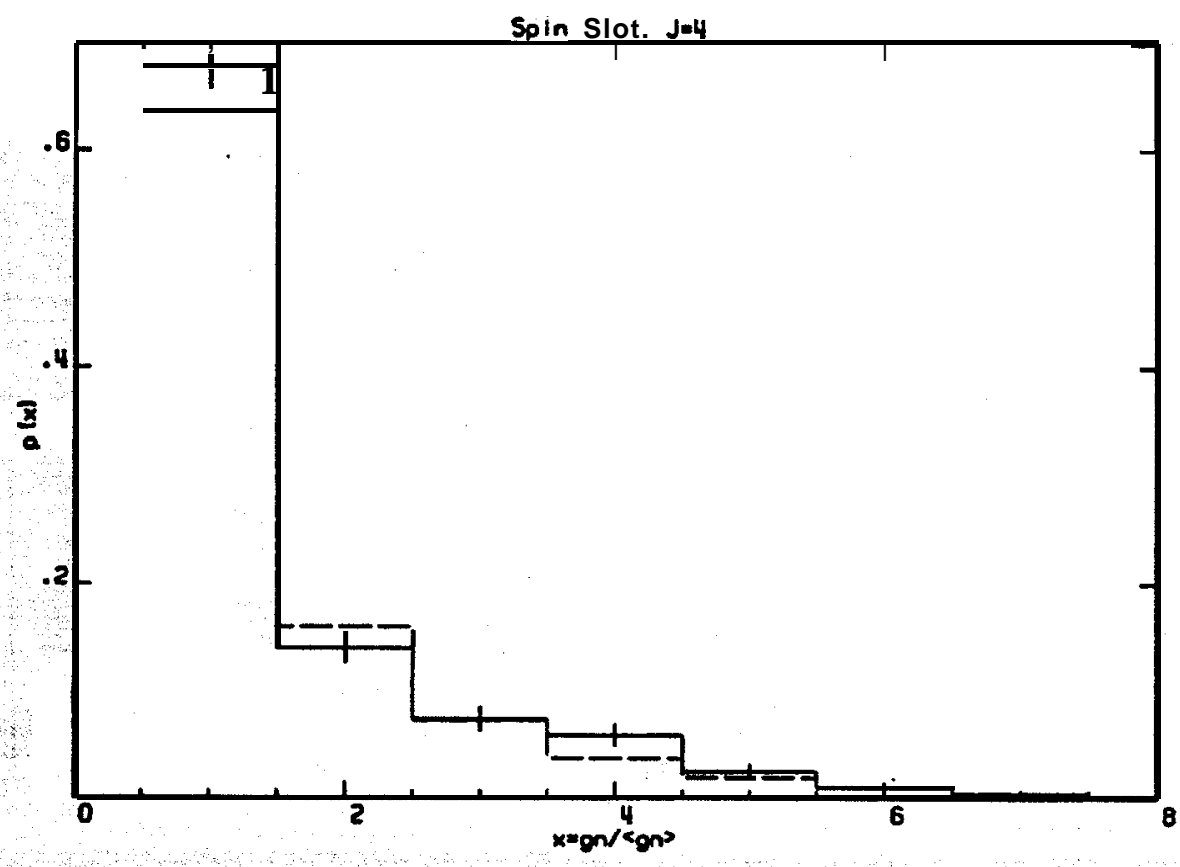

Fig. 4. Reduced neutron-width distribution for $J=4$. Calculations (solid line) compared with Porter-Thomas distribution (dashed line). 


\section{e. Fission-width distribution for spin group 1}

The output created in this run is shown in Table 5. The corresponding graphic output is given in Fig. 5. The FORODF sequence of statements used for generating the plot given in Fig. 5 is the following:

\section{$\mathrm{dvt} /$ hist /err3 /nodash f1s2se0ee8,/noerr /dash $0.2 \mathrm{f1s} 4$}

Table 5. Fission-width distribution with 4 degrees of freedom for $\mathrm{J}=3$

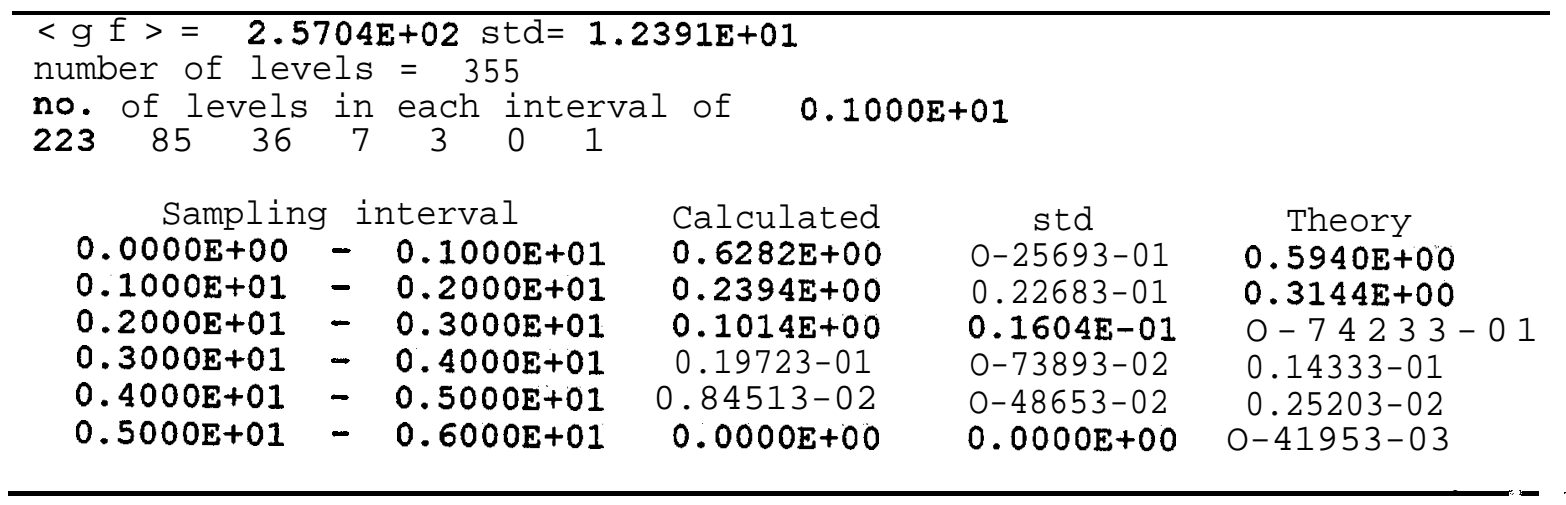

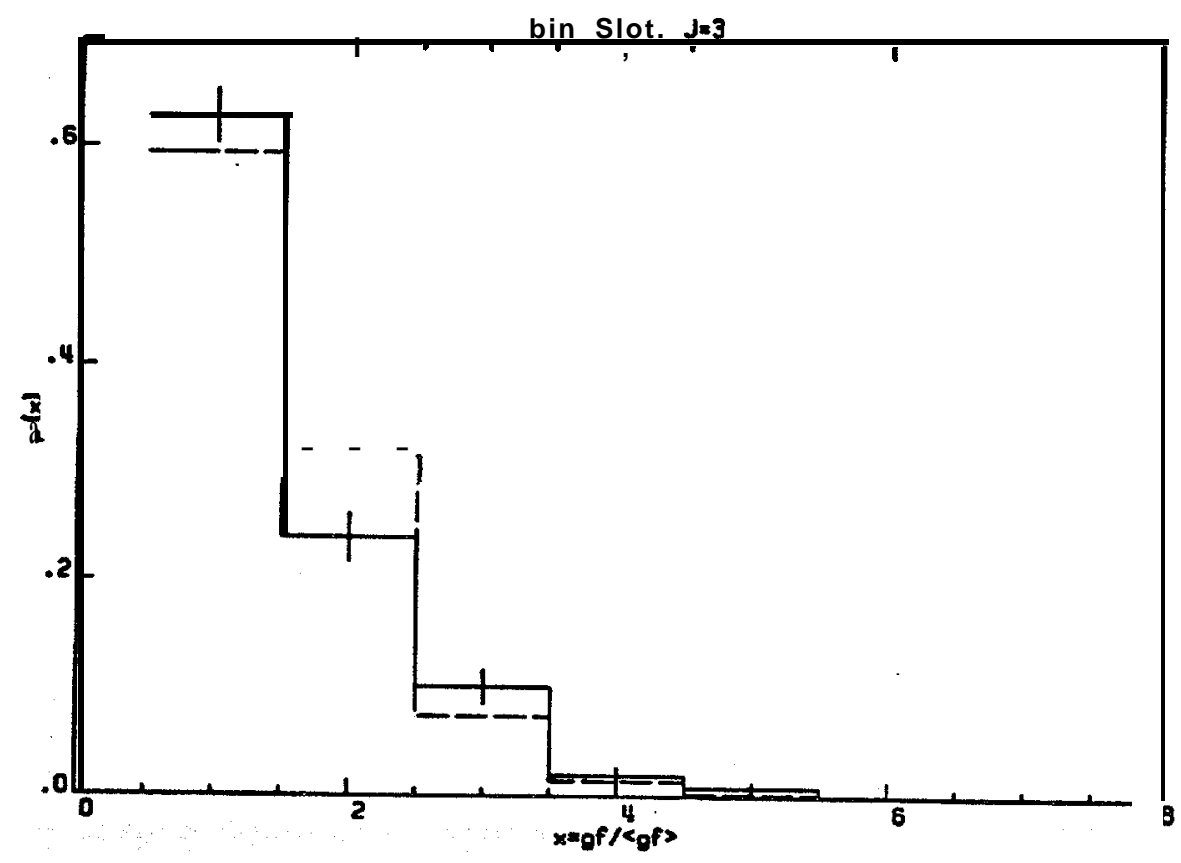

Fig. 5. Fission-width distribution for $\mathrm{J}=3$. Calculations (solid line) compared with $\chi^{2}$ distribution with 4 degrees of freedom (dashed line).' 


\section{f. Fission-width distribution for spin group 2}

The output created in this run is shown in Table 6. The corresponding graphic output is given in Fig. 6. The FORODF sequence of statements used for generating the plot given in Fig. 6 is the following:

$$
\mathrm{dvt} / \text { hist /err3 /nodash fl s2se0ee8,/noerr /dash } 0.2 \mathrm{fl} \mathrm{s4}
$$

Table 6. Fission-width distribution with 4 degrees of freedom for $\mathrm{J}=4$

\begin{tabular}{|c|c|c|c|c|}
\hline 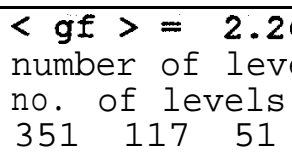 & $\begin{array}{l}689 \mathrm{E}+02 \text { std }=9 \\
\text { els }=549 \\
\text { in each inter } \\
21 \quad 7 \quad 2\end{array}$ & $\begin{array}{l}617 E+00 \\
1 \text { of } 0.10\end{array}$ & $E+01$ & \\
\hline $\begin{array}{l}\quad \text { Sampling } \\
0.0000 \mathrm{E}+00 \\
0.1000 \mathrm{E}+01 \\
0.2000 \mathrm{E}+01 \\
0.3000 \mathrm{E}+01 \\
0.4000 \mathrm{E}+01\end{array}$ & $\begin{array}{ll}\text { Interval } \\
=\quad 0.1000 \mathrm{E}+01 \\
=\quad 0.2000 \mathrm{E}+01 \\
=\quad 0.3000 \mathrm{E}+01 \\
-\quad 0.4000 \mathrm{E}+01 \\
=\quad 0.5000 \mathrm{E}+01\end{array}$ & $\begin{array}{l}\text { 'Calculated } \\
0.6393 \mathrm{E}+00 \\
0.2131 \mathrm{E}+00 \\
0.92903-01 \\
0.38253-01 \\
0.12753101\end{array}$ & $\begin{array}{c}\text { std } \\
0.2051 \mathrm{E}-01 \\
0.1749 \mathrm{E}-01 \\
0.1240 \mathrm{E}-01 \\
0.81933-02 \\
0.4793 \mathrm{E}-02\end{array}$ & $\begin{array}{c}\text { Theory } \\
0.5940 \mathrm{E}+00 \\
0.3144 \mathrm{E}+00 \\
0.7423 \mathrm{E}-01 \\
0.1433 \mathrm{E}-01 \\
0.2520 \mathrm{E}-02\end{array}$ \\
\hline
\end{tabular}

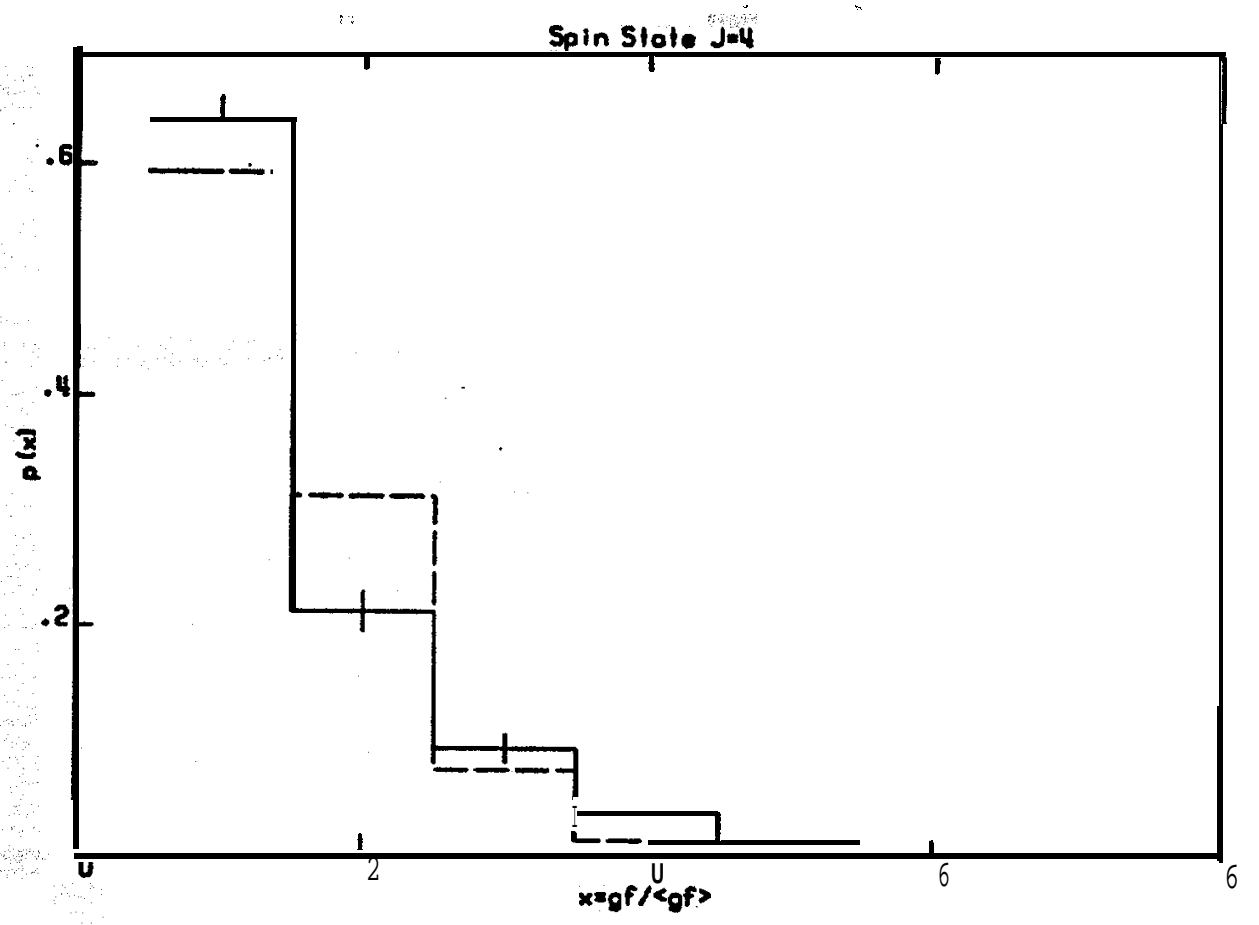

Fig. 6.' Fission-width distribution for $\mathrm{J}=4$. Calculations (solid line) compared with $\chi^{2}$ distnibution with 4 degrees of freedom (dashed line). 
g. $\underline{\Delta}_{3}$ statistic test for spin group 1

The output created in this run is shown in Table 7. The corresponding graphic output is given in Fig. 7. The FORODF sequence of statements used for generating the plot given in Fig. 7 is the following:

\section{dvt /nohist fl s2se0ee500,/hist fl s3}

Table 7. The A, results for $\mathrm{J}=3$ (only the first $30{ }^{235} \mathrm{U}$ s-wave resonances are shown)

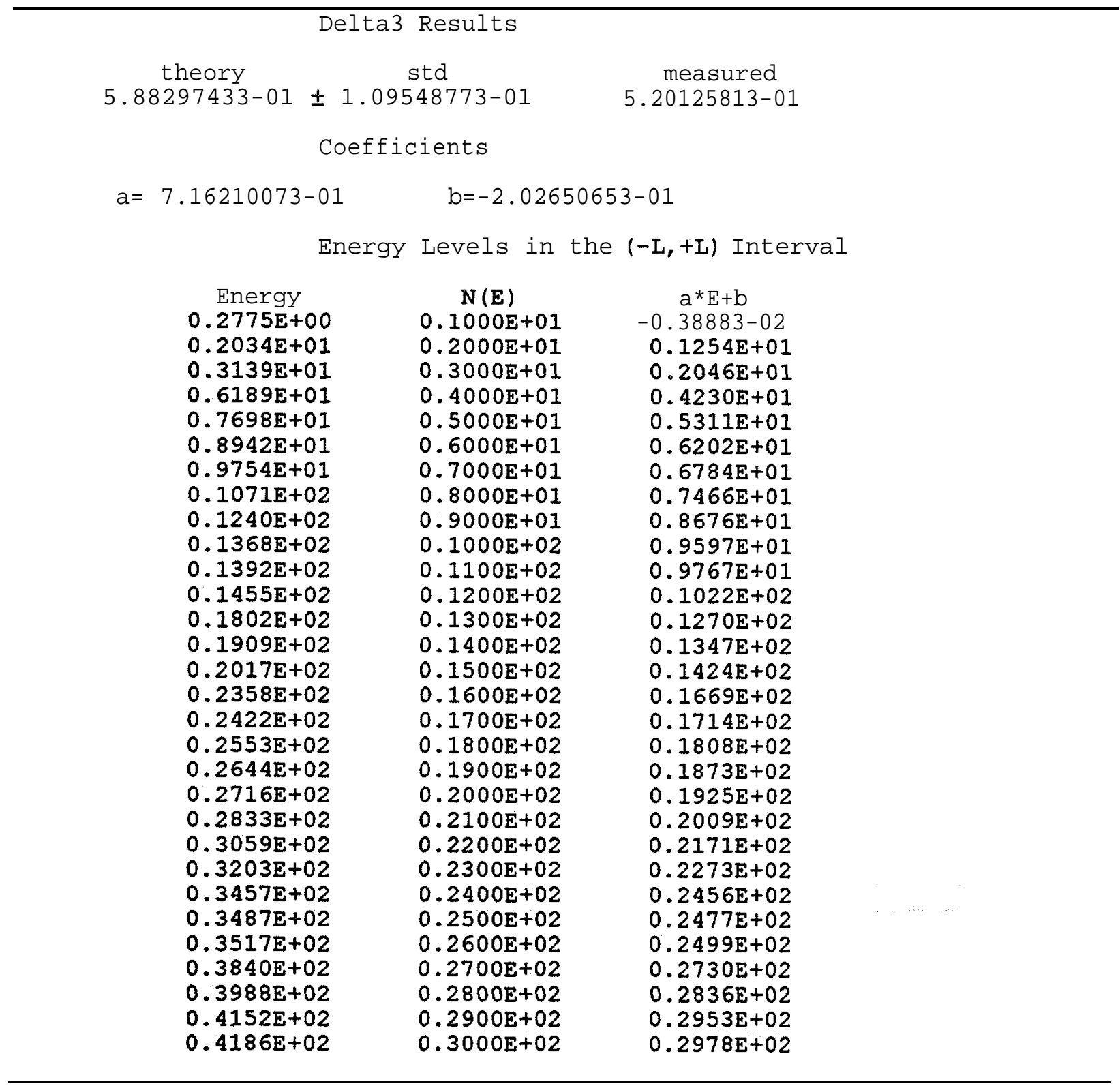




\section{3}

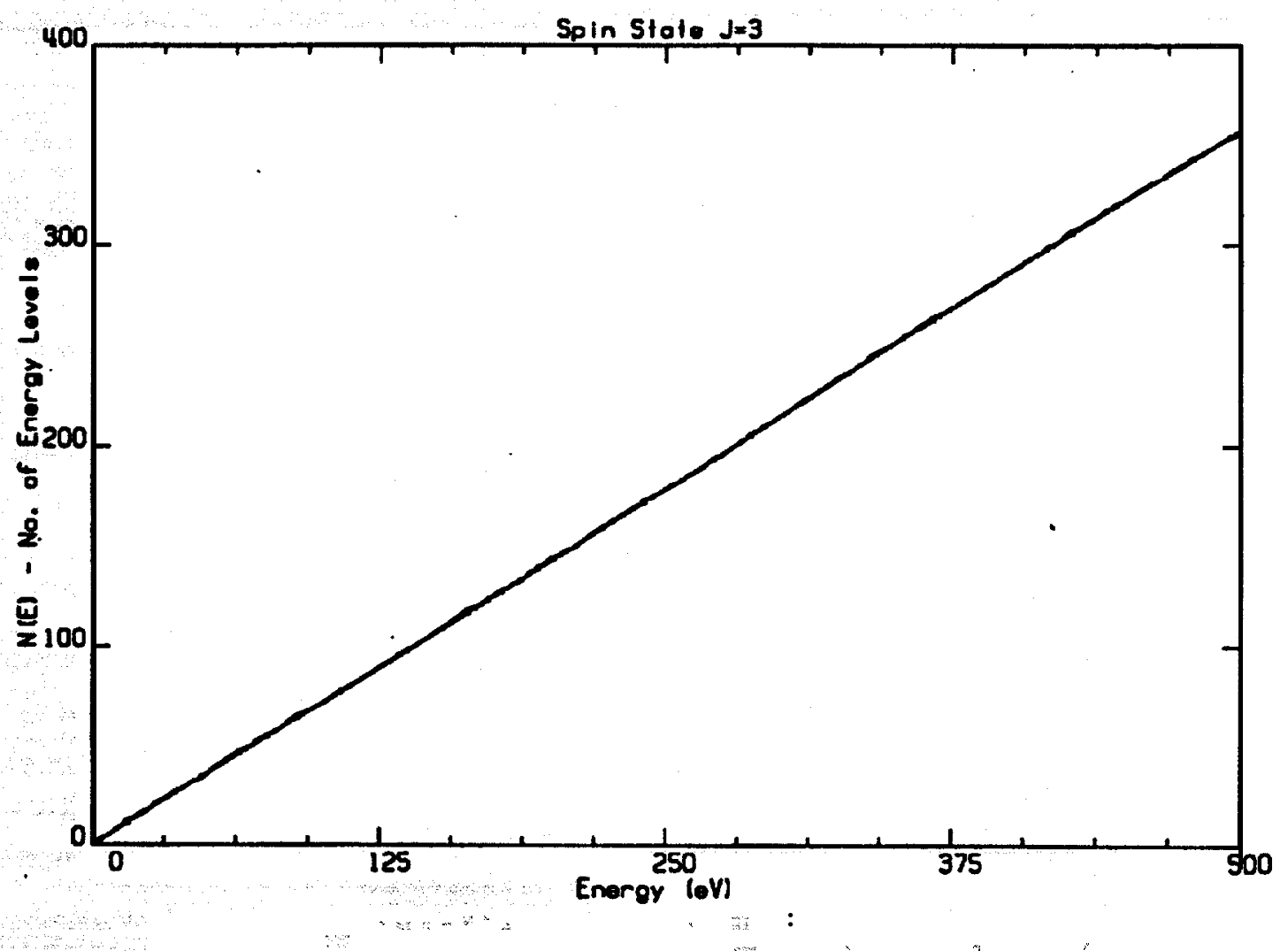

Fig. 7. Cumulative number of energy levels vs energy for $J=3$.

\section{h. $\underline{\Delta}_{3}$ statistic test for spin group 2}

The output created in this run is shown in Table 8. The corresponding graphic output is given in Fig. 8. The FORODF sequence of statements used for generating the plot given in Fig. 8 is the following:

$\mathrm{dvt} /$ nohist fl s2se0ee500,/hist fl s3 
Table 8. The A, results for $\mathrm{J}=4$ (only the first $30{ }^{235} \mathrm{U}$ s-wave resonances are shown)

Delta3 Results

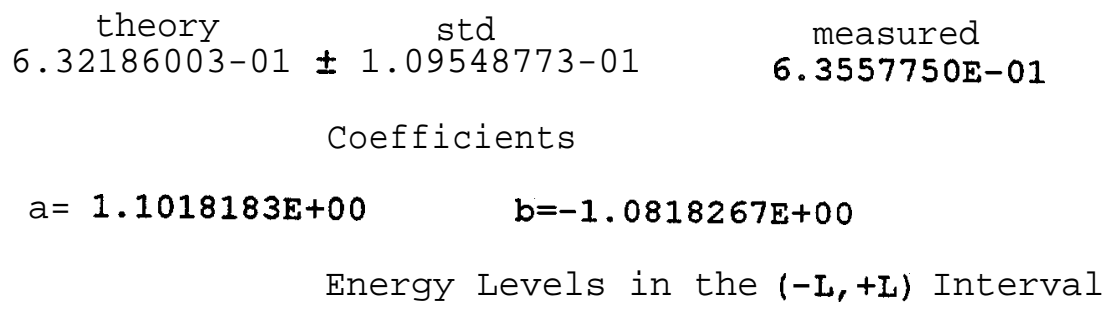
Energy
$0.1133 \mathrm{E}+01$
$N(E)$
$a \star E+b$
$0.2777 \mathrm{E}+01$
$0.1000 \mathrm{E}+01$
$0.1663 \mathrm{E}+00$
$0.3614 E+01$
$0.2000 \mathrm{E}+01$
$0.1978 \mathrm{E}+01$
$0.4852 \mathrm{E}+01$
$0.3000 E+01$
$0.2900 \mathrm{E}+01$
$0.5438 \mathrm{E}+01$
$0.4000 \mathrm{E}+01$
$0.4264 \mathrm{E}+01$
$0.6393 E+01$
$0.5000 \mathrm{E}+01$
$0.4910 \mathrm{E}+01$
$0.7079 \mathrm{E}+01$
$0.6000 \mathrm{E}+01$
$0.5962 \mathrm{E}+01$
$0.8767 \mathrm{E}+01$
$0.7000 \mathrm{E}+01$
$0.6718 \mathrm{E}+01$
$0.9277 \mathrm{E}+01$
$0.8000 \mathrm{E}+01$
$0.1016 \mathrm{E}+02$
$0.9000 E+01$
$0.8578 \mathrm{E}+01$
$0.1000 \mathrm{E}+02$
$0.9140 \mathrm{E}+01$
$0.1167 \mathrm{E}+02$
$0.1100 \mathrm{E}+02$
$0.1012 \mathrm{E}+02$
0. $1240 \mathrm{E}+02$
$0.1200 \mathrm{E}+02$
0. $1286 \mathrm{E}+02$
$0.1300 \mathrm{E}+02$
$0.1327 \mathrm{E}+02$
$0.1400 \mathrm{E}+02$
$0.1411 \mathrm{E}+02$
$0.1500 E+02$
$0.1541 \mathrm{E}+02$
$0.1600 \mathrm{E}+02$
$0.1609 \mathrm{E}+02$
$0.1700 \mathrm{E}+02$
$0.1664 \mathrm{E}+02$
$0.1800 \mathrm{E}+02$
$0.1803 \mathrm{E}+02$
$0.1900 \mathrm{E}+02$
$0.1900 \mathrm{E}+02$
$0.2000 E+02$
$0.1929 \mathrm{E}+02$
$0.2063 E+02$
$0.2100 \mathrm{E}+02$
$0.2107 \mathrm{E}+02$
$0.2200 \mathrm{E}+02$
$0.2300 \mathrm{E}+02$
$0.2293 E+02$
$0.2400 \mathrm{E}+02$
$0.2341 E+02$
$0.2435 \mathrm{E}+02$
$0.2500 \mathrm{E}+02$
$0.2600 \mathrm{E}+02$
$0.2499 \mathrm{E}+02$
$0.2649 \mathrm{E}+02$
$0.2778 \mathrm{E}+02$
$0.2813 E+02$
$0.2700 \mathrm{E}+02$
$0.2800 \mathrm{E}+02$
$0.2900 \mathrm{E}+02$
$0.1177 \mathrm{E}+02$
$0.1258 \mathrm{E}+02$
$0.1309 \mathrm{E}+02$
$0.1354 \mathrm{E}+02$
$0.1447 \mathrm{E}+02$
$0.1590 \mathrm{E}+02$
$0.1664 \mathrm{E}+02$
$0.1725 \mathrm{E}+02$
$0.1878 \mathrm{E}+02$
$0.1985 \mathrm{E}+02$
$0.2018 E+02$
$0.2165 \mathrm{E}+02$
$0.2213 \mathrm{E}+02$
$0.2418 \mathrm{E}+02$
$0.2472 \mathrm{E}+02$
$0.2575 \mathrm{E}+02$
$0.2645 \mathrm{E}+02$
$0.2810 \mathrm{E}+02$
$0.3000 E+02$
$0.2953 \mathrm{E}+02$
$0.2992 E+02$ 


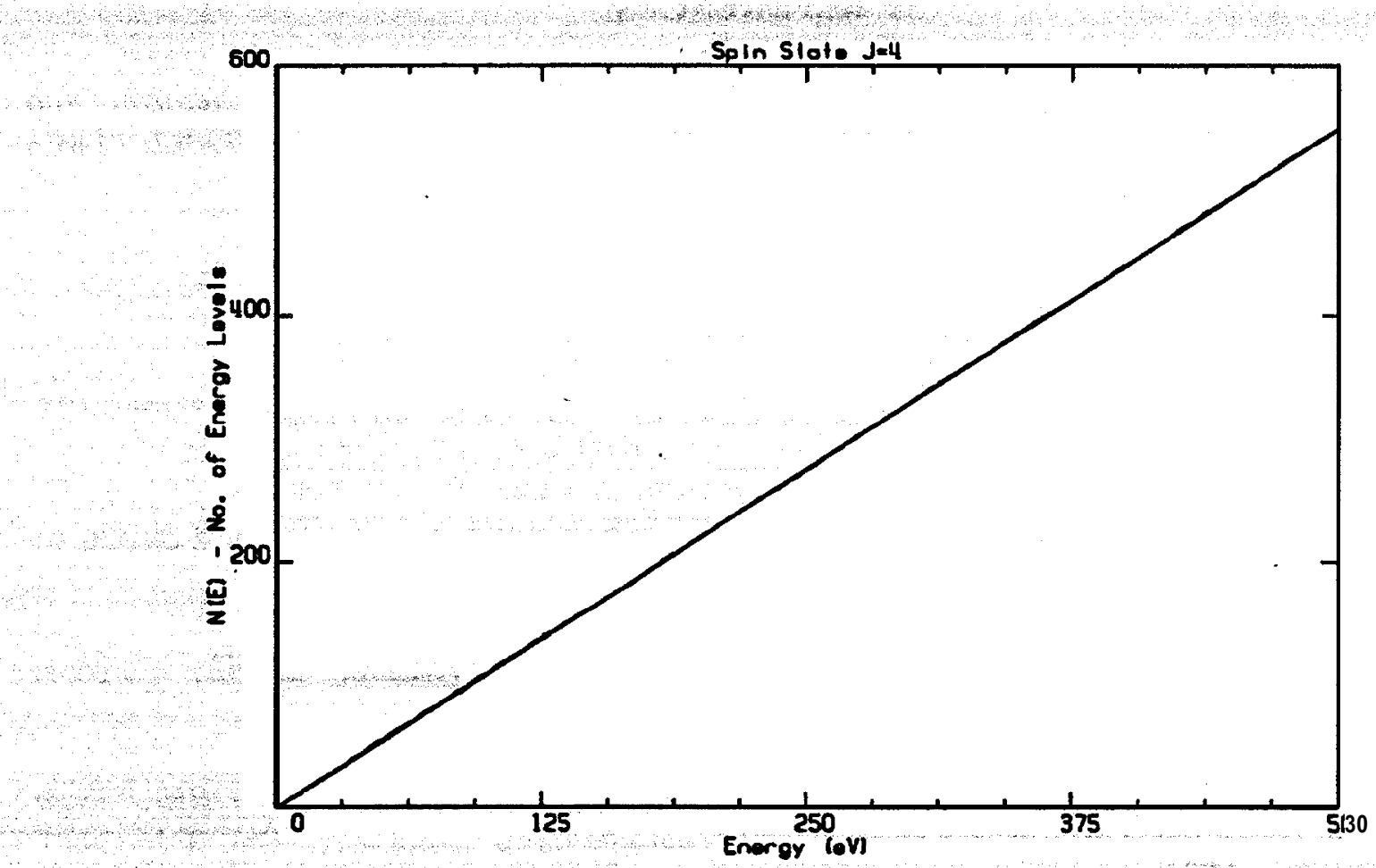

Fig. 8. Cumulative number of energy levels vs energy for $\mathrm{J}=\mathbf{4}$. 


\section{REFERENCES}

1. N. M. Larson, Update Users 'Guide for SAMMY: Multilevel R-matrix Fits to Neutron Data Using Bayes ' Equations, ORNL/TM-97101/R1 (1985) and ORNL/TM-9179/R2, Martin Marietta Energy Systems, Inc., Oak Ridge Natl. Lab., 1989.

2. E. P. Wigner, Conf. On Neutron Physics by Time-of-Flight, Gatlinburg, Term., 1956, ORNL2309, Union Carbide Corp., Nucl. Div., Oak Ridge Natl. Lab., 1957, p. 59.

3. C. E. Porter and R. G. Thomas, "Fluctuations of Nuclear Reaction Widths," Phys. Rev. 104, 483-491 (1956).

4. F. J. Dyson and M. L. Mehta., "Statistical Theory of the Energy Levels of Complex Systems," J.Math. Phys. 4,701 (1963).

5. Dennis Wolfe, Computer Science and Mathematics Division, ORNL, personal communication, August 1995.

6. J. G. Craven, OPRODF, A DECsystem-10 Data Manipulation Program for ORELA Data Formatted Files, ORNL/CSD/TM-45, Union Carbide Corp., Nucl. Div., Oak Ridge Natl. Lab., May 1978.

7. L. C. Leal, G. de Saussure, and R. B. Perez, "An R-Matrix Analysis of the ${ }^{235} \mathrm{U}$ Neutron Induced Cross Sections up to $500 \mathrm{eV,"} \mathrm{Nucl.} \mathrm{Sci.} \mathrm{Eng.} \mathrm{109,} \mathrm{1-17} \mathrm{(1991).}$ 


\section{APPENDIX A}

Listing of the SAMDIST code written in FORTRAN 77 language on the IBM RISC6000 platform.

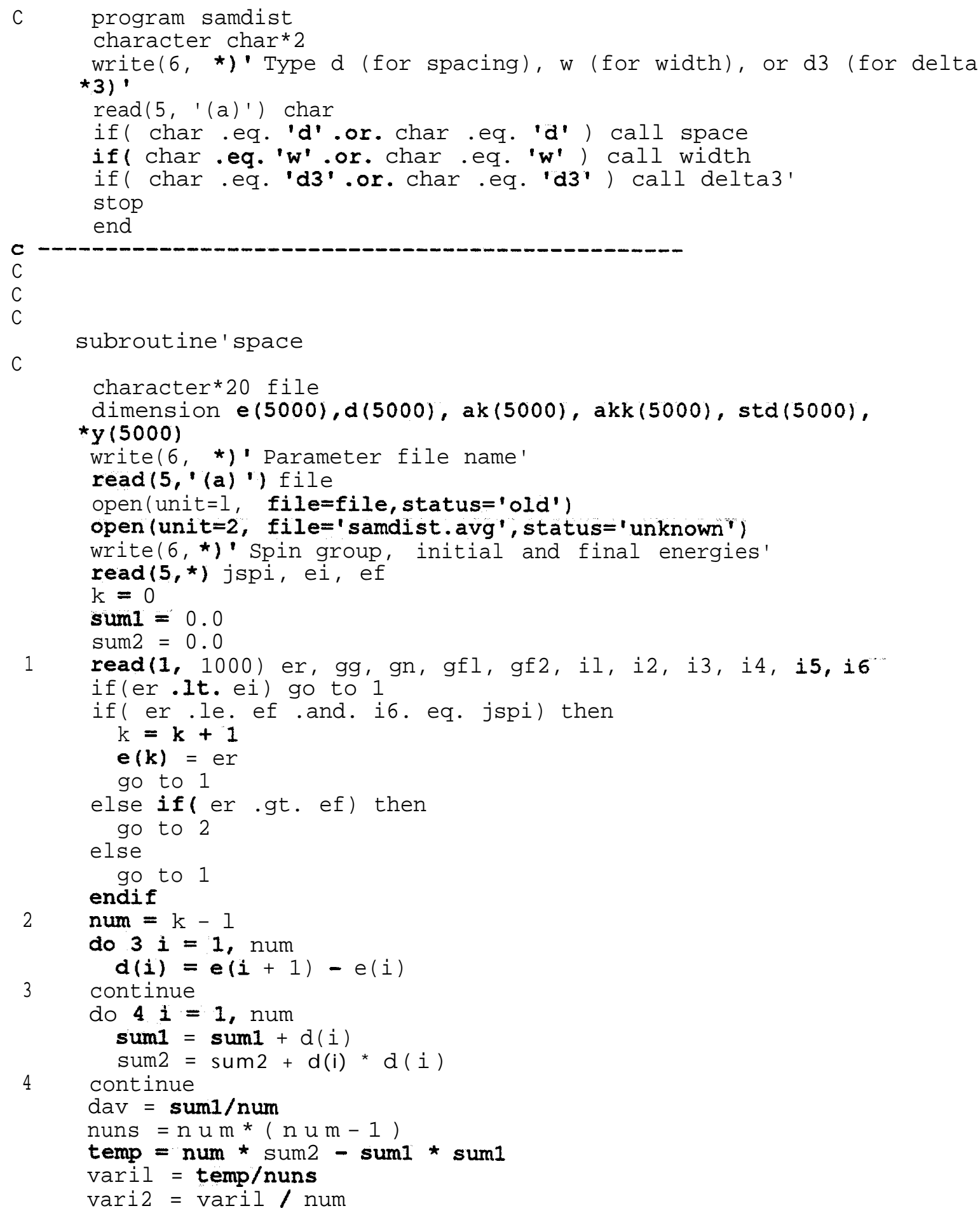




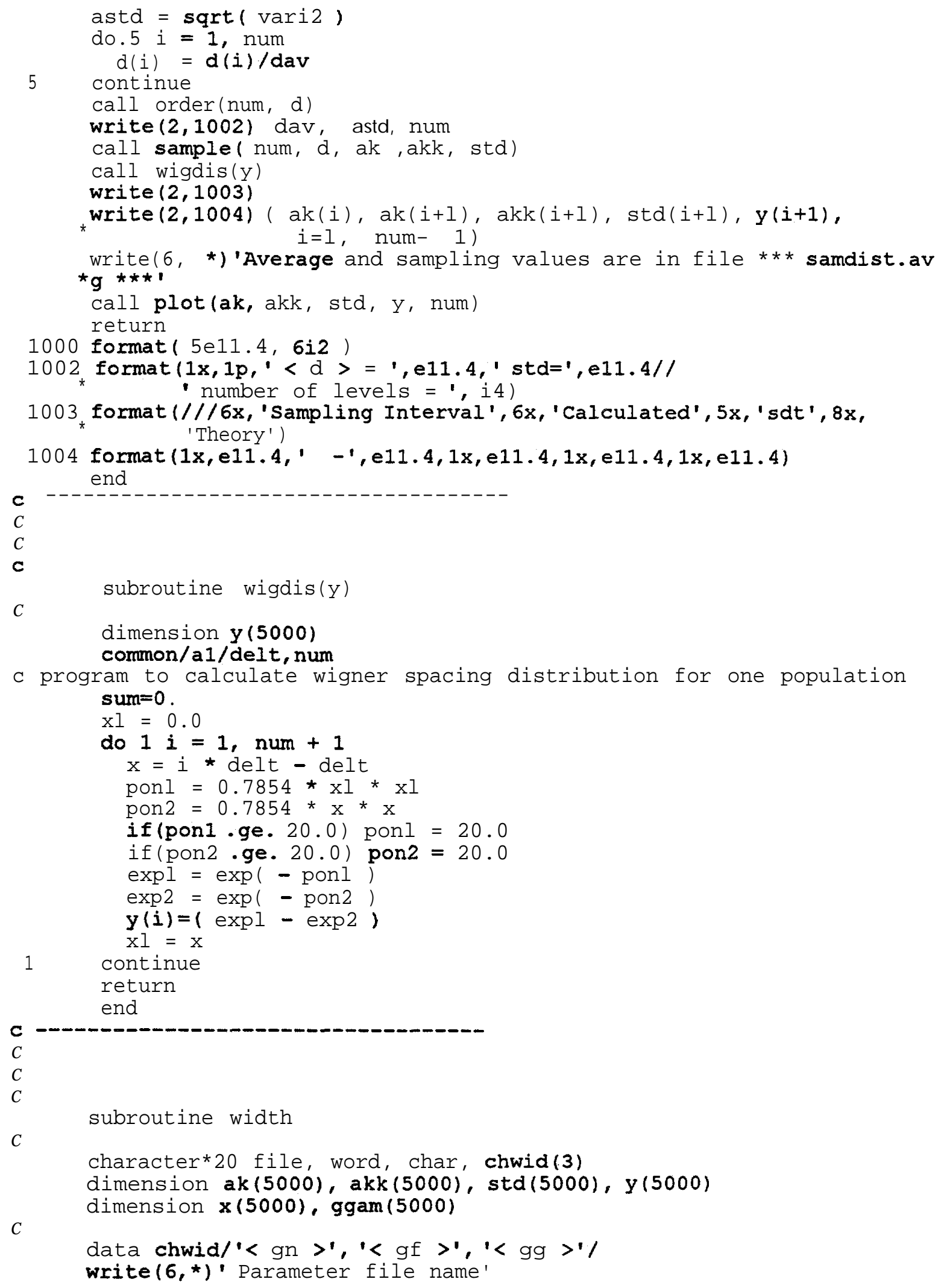




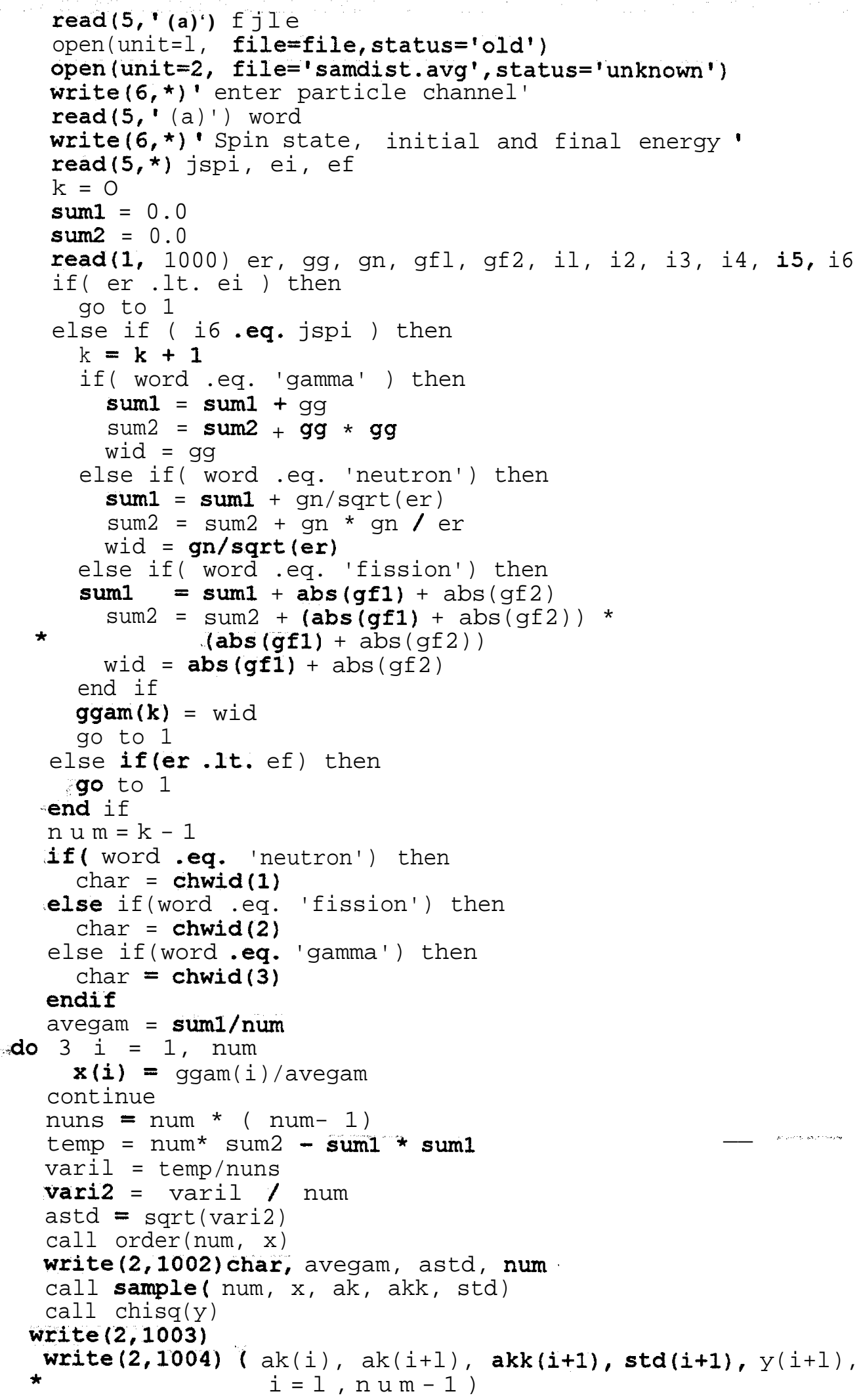




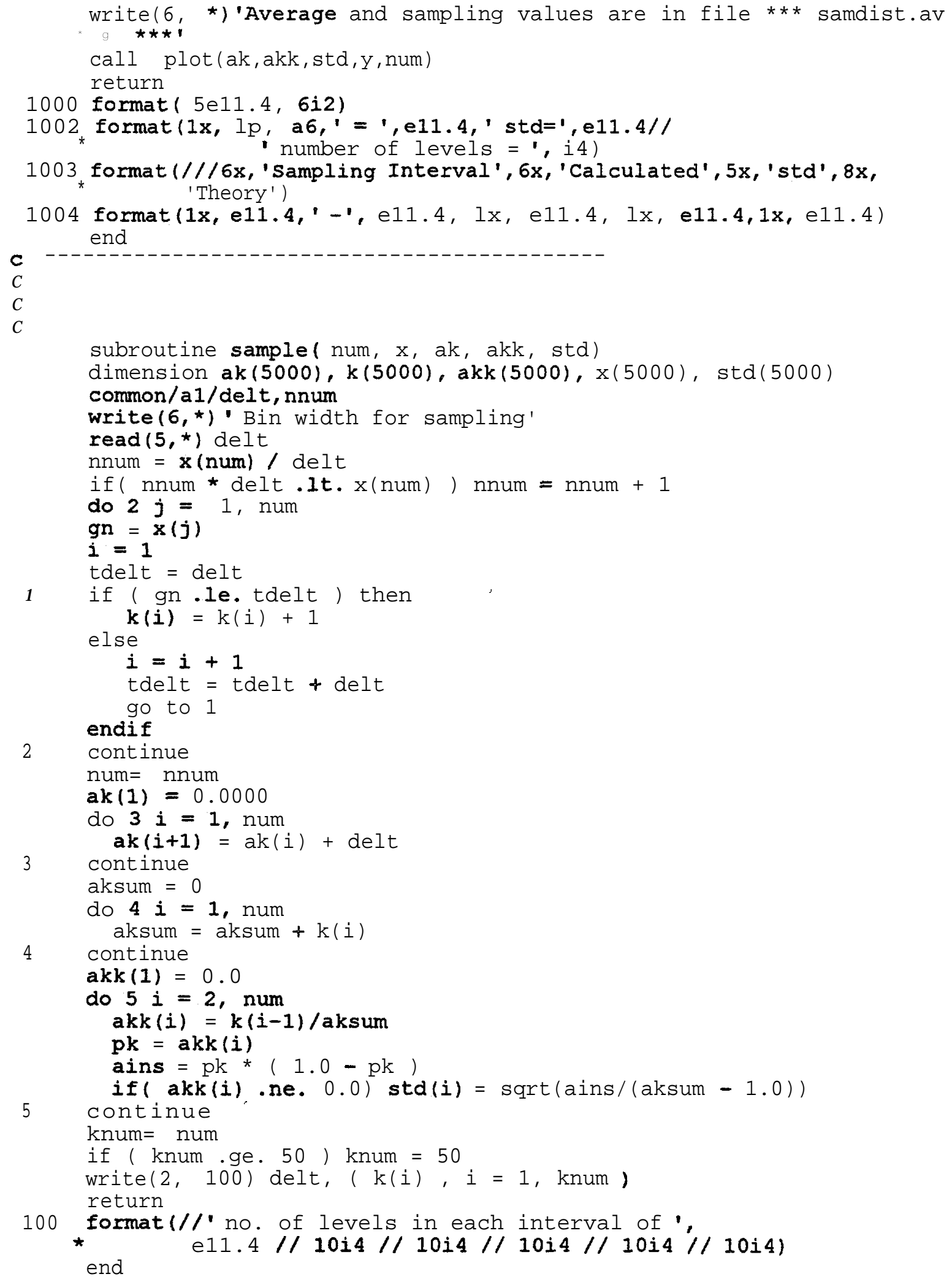


c

$c$

C

subroutine chisq(yy)

dimension yy (5000)

common/rq/xf

common/a1/del, $\mathrm{n}$

external chipdf

write $(6, *)$ ' Degrees of freedom:'

$\operatorname{read}(5, *) \mathrm{df}$

$\mathrm{xf}=\mathrm{df}$

del $z=$ del

$\mathrm{zl}=0.0$

$Y Y(1)=0.0$

do $10 i=1, n$

$z u=\operatorname{del} z *$ float $(i)$

call rqg7 (zl, zu, chipdf,y)

$\mathrm{yy}(i+1)=y$

$\mathbf{z} \mathbf{l}=\mathbf{z u}$

10 continue

return

end

function chipdf(z)

C

C

common/rq/df, p1, p2, p3

$z=d f \star z$

$\mathrm{dfh}=\mathrm{df} / 2.0$

edf $h=d f h-1.0$

call gamma (df, gam)

$c=\operatorname{dfh} /((2.0 * \star$ edfh $) *$ gam $)$

chipdf $=c^{*}\left(z^{\star \star} \operatorname{edfh}\right) * \exp (-z / 2.0)$

return

end

c

c

$c$

subroutine $\operatorname{rqg} 7(x \mathbf{l}, \mathbf{x u}, \mathbf{f c t}, \mathrm{y})$

common/rg/parm1, parm2, parm3, parm4, parm5

c

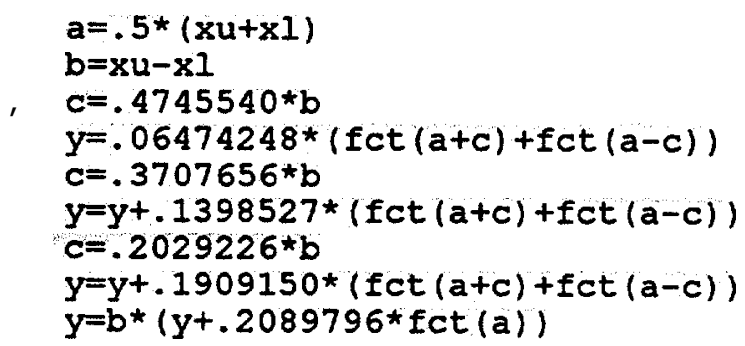




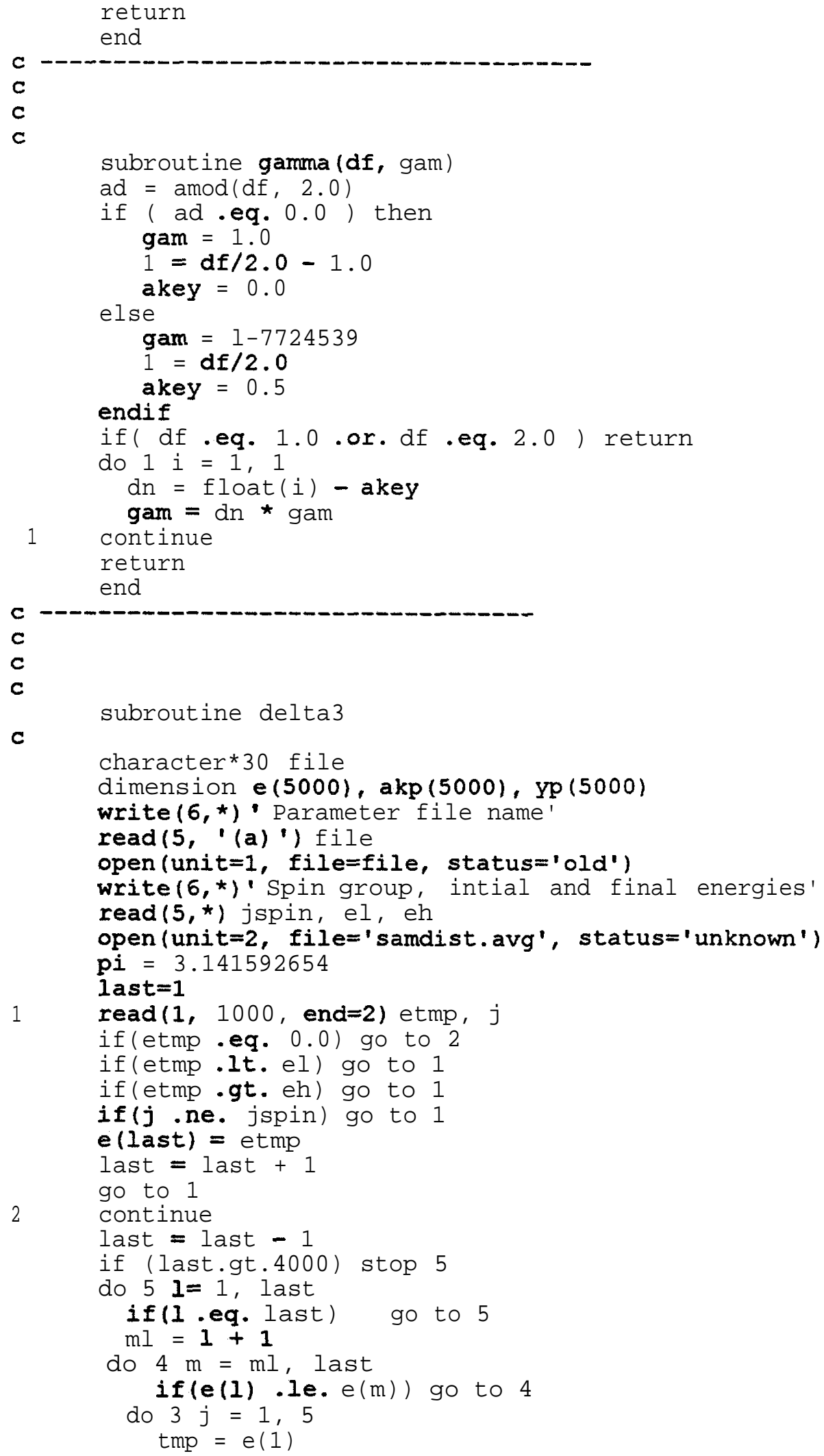




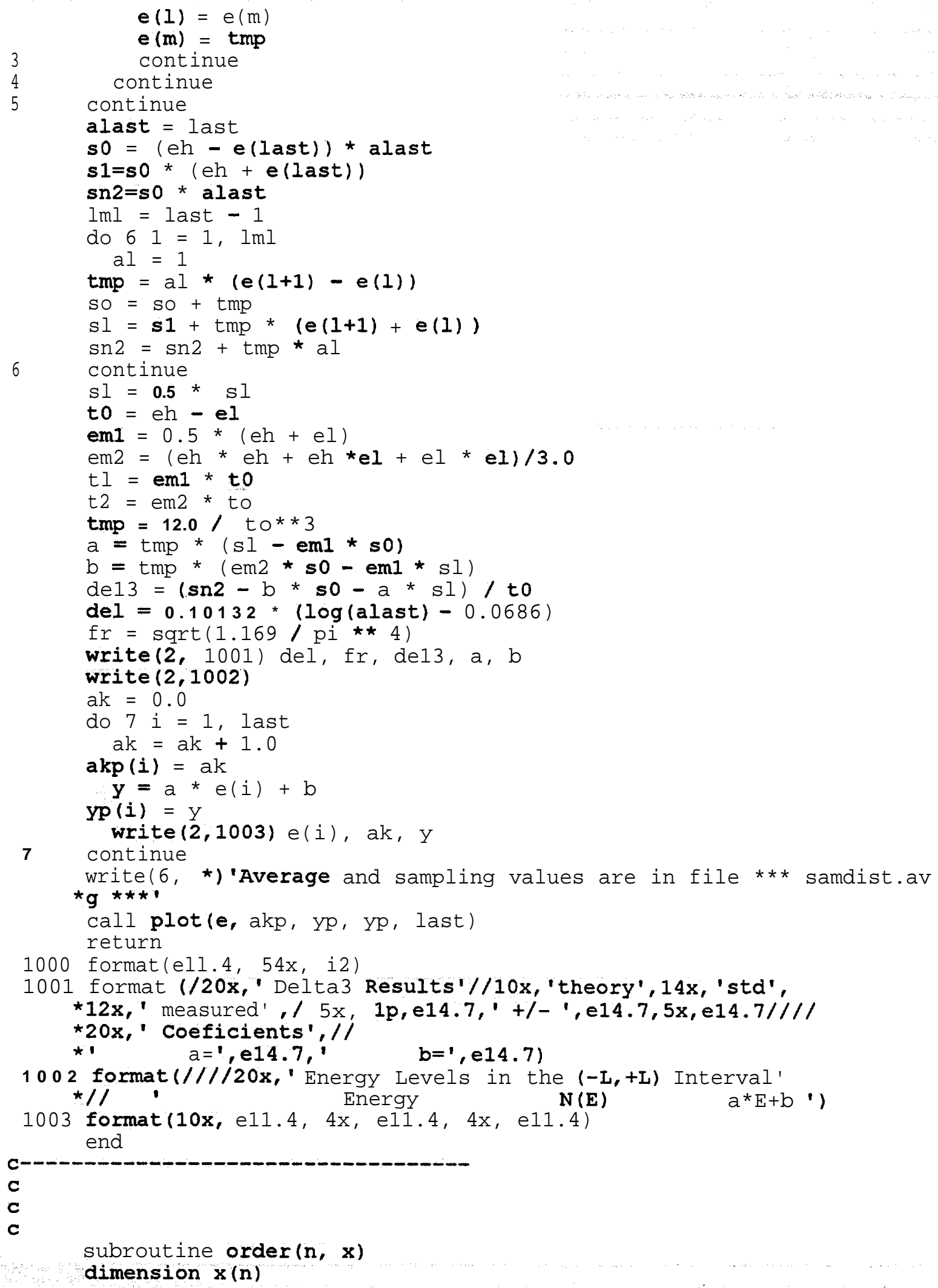




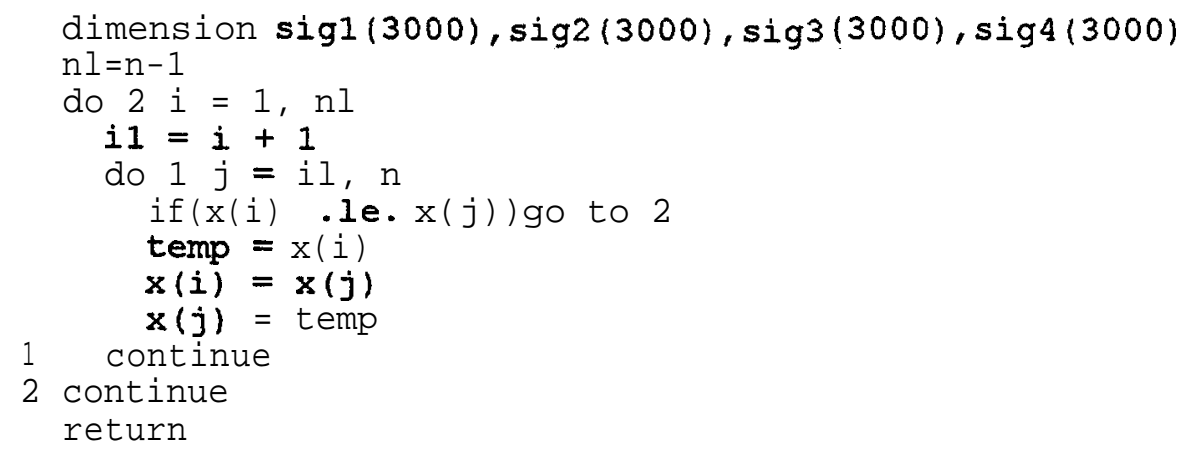




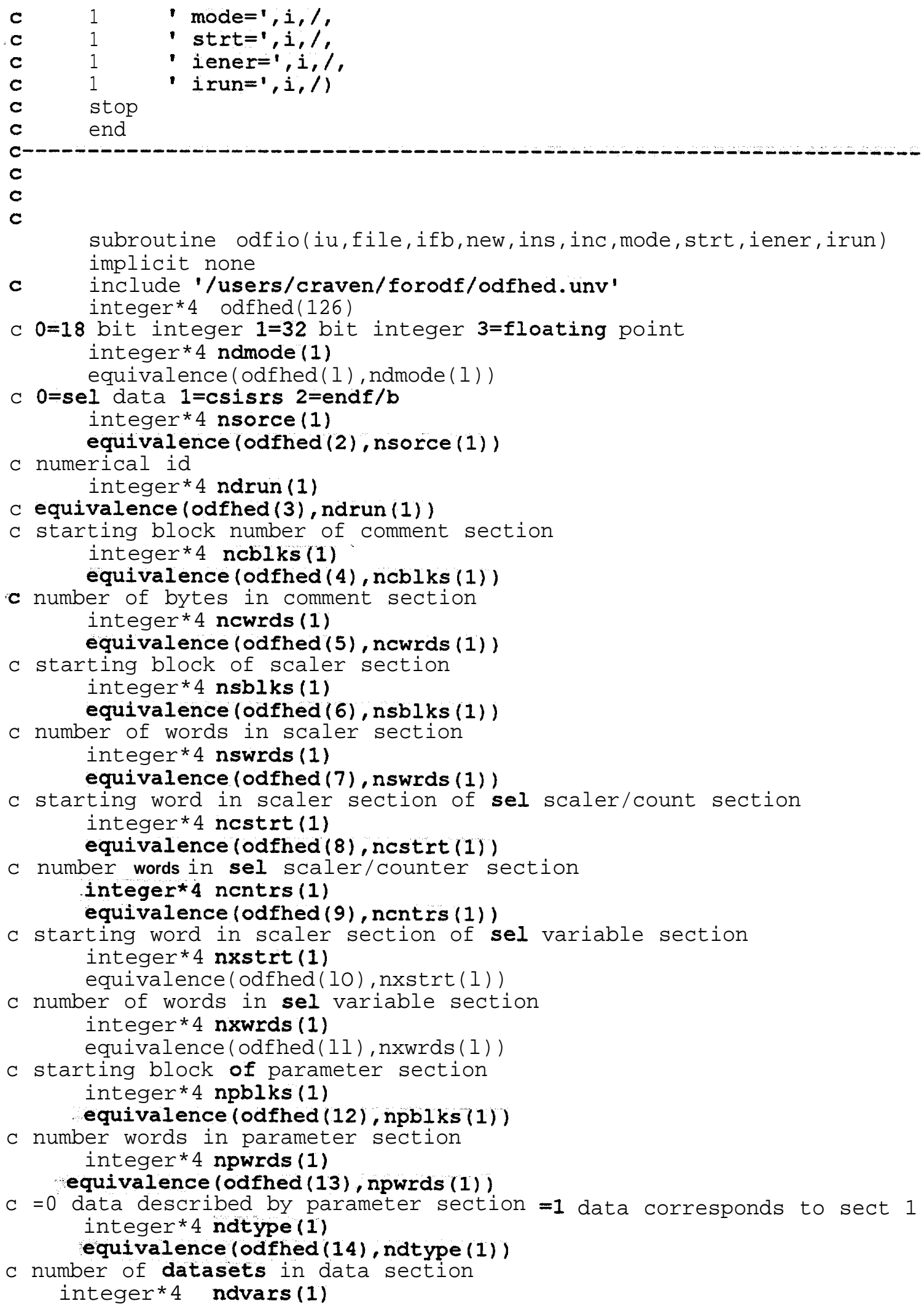


equivalence (odfhed (15), ndvars (1))

C starting block of data section

integer 4 ndblks (1)

equivalence (odfhed (16), ndblks (1))

$c$ number of words in each dataset

integer 4 ndwrds (1)

equivalence (odfhed (17), ndwrds (1))

$c$ endf/b designation (charge, mass)

integer*4 ndzan (1)

equivalence (odfhed (18), ndzan(1))

$c$ endf/b ratio nuclear mass to neutron integer* 4 ndawr (1)

equivalence (odfhed (19), ndawr (1))

$c$ endf/b number assigned by national neutron cross section center integer* 4 ndmat (1)

equivalence (odfhed (20), ndmat (1))

$c$ endf/b file number

integer* 4 ndmf (1)

equivalence (odfhed (21), ndmf(1))

$c$ endf/b reaction type number

integer* 4 nomt (1)

equivalence (odfhed (22), ndmt (1))

$c$ if ndtype=1 then ndvswt $=0$ engery decreases, $=1$ increases integer 4 ndvswt (1)

equivalence (odfhed (23), ndvswt (1))

$\mathrm{c}=1$ data dead time created, $=0$ not

integer* 4 nddswt (1)

equivalence (odfhed (24), nddswt (1))

C starting word of data from mode 0

integer* 4 ndstrt (1)

equivalence (odfhed (25), ndstrt (1))

c last word written of parameter section

integer* 4 ndwend

equivalence (odfhed (26), ndwend)

c words 27 through 126 is energy index table,

C largest energy for each $n$ blocks, $n=($ ndwrds $/ 125)+1$ real*4 ndtabl (100)

equivalence (odfhed (27), ndtabl (1))

c starting block number of comment section

integer iu, ifb, new, ins, inc, mode, strt, iener, irun, iarray (1)

integer ibuf4(126)

integer $* 4 i, j, k, l$, zero, $i b l k, i b c, i l c, i s c, i s n, i n d e x, j u n k, i w o r d 4$

integer* 4 iii, system

integer*2 ibuf2 (252), xword4 (2), iword2

logical*4 ex

character* $(*)$ file

character commd*3, fcommd*252

equivalence (xword4 (1), iword4), (xword4 (1), iword2)

equivalence (ibuf2 (1), odfhed), (ibuf4 (1), odfhed)

data commd/'rm '/

data zero/o/

c

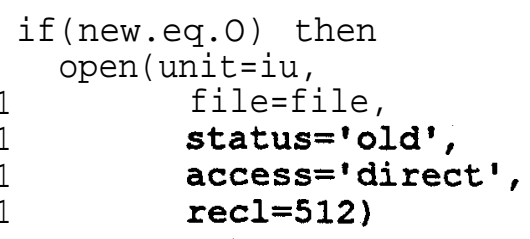


else

inquire (file=file, exist=ex)

if (ex) then

fcommd=commd//file//char (0)

iii $=$ system (fcommd)

endif

open (unit=iu,

1 file=file,

status=' unknown',

access='direct',

go to 12

c

endif

$c$

endif

C

read (iu, rec $=1$ ) odfhed

ins=ndvars $(1)$

i fb=ndblks (1)

inc=ndwrds (1)

mode $=$ ndmode (1)

strt=ndstrt (1)

iener $=0$

if (ndtype (1) .ne.0) iener $=-1$

irun=ndrun (1)

$j=125$

if (mode. eq. 0 ) $j=250$

$i=(i n c-1) / j$

if $(i * j$. ne.inc) $i=i+1$

$i b l k=i f b+(i * i n s)-1$

read ( $i u$, rec $=i b l k$, err $=1$ ) odfhed

return

write (iu, rec=iblk) odfhed

return

entry outodf (iu, ifb, ins, isn, mode, strt, isc, inc, iarray, index)

if (ins.le.0) go to 14

if(isn.le.0)go to 14

if (isn.gt.ins)go to 14

if(isc.le.0)go to 14

if(inc.le.0)go to 14

$i b c=1$

ilc=inc

if (mode.eq. 0 ) go to 23

iblk $=($ isc- 1$) / 125$

$i=i s c-(i b l k * 125)$

$i b l k=(i b l k * i n s)+i f b+i s n-1$

if (i.eq.l)go to 3

read ( $i u, r e c=i b l k$ ) ibuf 4

$l=i+i l c-1$

if (1.gt. 125) $1=125$

do $2 j=i+1, i+1$

ibuf $4(j)=i$ array (ibc)

$2 \quad i b c=i b c+i n d e x$

write (iu, rec=iblk) odfhed

ilc $=i l_{c}-(1-i+1)$

if (ilc.eq.0) return

.iblk=iblk+ins

$3 \quad i=i l c / 125$ 
if(i.eq.0)go to 5

do $4 j=i b c, i b c-1+(i * 125 * i n d e x), 125 * i n d e x$

write (iu, rec=iblk) zero, (iarray $(k), k=j, j+(125 * i n d e x), i n d e x)$

$i b l k=i b l k+i n s$

ibc $=i b c+(i * 125 *$ index $)$

ilc $=i l c-(i * 125)$

if (ilc.eq. 0 ) return

$5 \operatorname{read}(i u, r e c=i b l k) i b u f 4$

do $6 j=2$, ilct

ibuf $4(j)=i a r r a y(i b c)$

$6 \quad i b c=i b c+i n d e x$

write (iu, rec=iblk) ibuf 4

return

entry inodf (iu, ifb, ins, isn, mode, strt, isc, inc, iarray, index)

if (ins.le.0) go to 16

if(isn.le.0)go to 16

if (isn.gt.ins)go to 16

if (isc.le.0)go to 16

if (inc.le.0)go to 16

$i b c=1$

ilc=inc

if (mode.eq. 0 ) go to 20

iblk $=($ isc- 1$) / 125$

$i=i s c-(i b l k * 125)$

$i b l k=(i b l k * i n s)+i f b+i s n-1$

if (i.eq.1)go to 8

read ( $i u$, rec $=i b l k) i b u f 4$

$i b l k=i b l k+i n s$

$l=i+i l c-1$

if (l.gt. 125) $1=125$

do $7 \quad j=i+1,1+1$

iarray (ibc) $=$ ibuf $4(j)$

$7 \quad i b c=i b c+i n d e x$

$i l c=i l c-(1-i+1)$

if (ilc.eq. 0 ) return

$8 \quad i=i l c / 125$

if(i.eq. 0$)$ go to 10

do $9 j=i b c, i b c-1+(i \star 125 * i n d e x), 125 * i n d e x$

9 iblk=iblk+ins

$i b c=i b c+(i \star 125 * i n d e x)$

ilc $=i l c-(i * 125)$

if (ilc.eq. 0 ) return

$10 \operatorname{read}(i u, r e c=i b l k)$ ibuf 4

do $11 j=2, i l c+1$

iarray $(i b c)=i b u f 4(j)$

11 ibc=ibctindex

return

12 do $13 \quad i=1,126$

$13 \operatorname{odfhed}(i)=0$

if (mode.eq.0.and.iener.ne.0) go to 28

if (mode.eq. 0. and.ins.ne.1) go to 28

if (mode.ne.0.and.strt.ne.0)go to 28

if (strt.1t.0)go to 28

ndmode (1) =mode

ndrun $(1)=$ irun

ndwrds $(1)=$ inc

ndvars $(1)=$ ins 


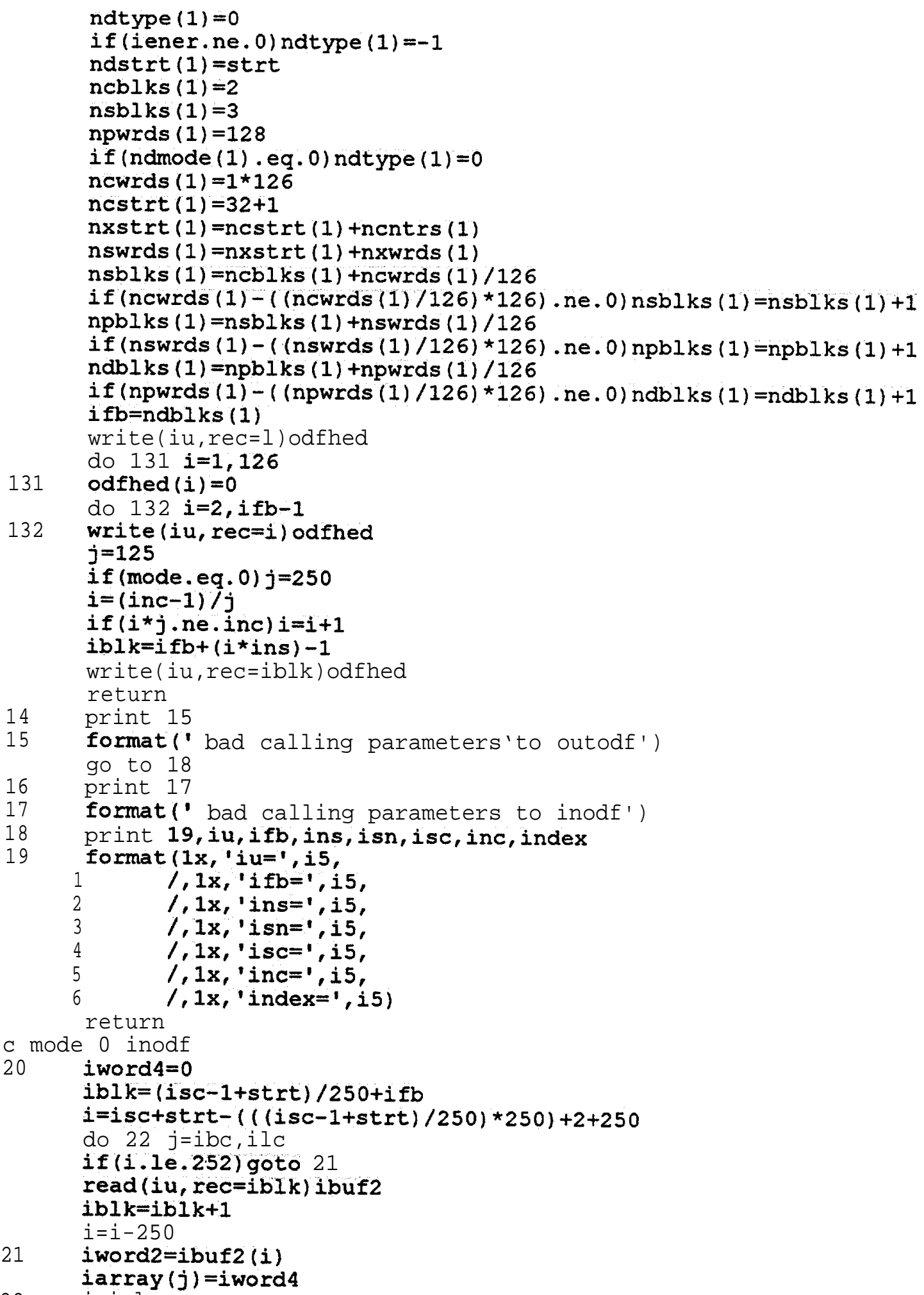


23 iblk=(isc-1+strt) $/ 250+i f b$

read (iu, rec $=i b l k$, err $=24$ ) ibuf2

$24 i=i s c+s t r t-(((i s c-1+s t r t) / 250) \star 250)+2$

do $27 \mathrm{j}=\mathrm{ibc}$, ilc

if (i.le.252) goto 26

write ( $i u$, rec=iblk) ibuf2

$i b l k=i b l k+1$

read (iu, rec=iblk, err=25) ibuf2

$25 \quad i=i-250$

$26 \quad i w o r d 4=i \operatorname{array}(j)$

ibuf2 $(i)=i$ word2

$27 \quad i=i+1$

write (iu, rec $=i b l k$ ) ibuf2

return

28 print 29, iu, file, ifb, new, ins, inc, mode, strt, iener, irun

29 format (' bad calling parameters to odfio',

$9 \quad /$ 'iu=',i5,

$1, '$ file=', a10,

l,' ifb=', i5,

1, ' new $=1, i 5$,

1.' ins $=1$, i5,

1, 'inc=', i5,

1,' mode=', i5,

1, ' strt=1, i5,

1.' iener $=1, i 5$,

1, irun=', i5)

return

end 


\section{APPENDIX B}

Listing of the ${ }^{235} \mathrm{U}$ s-wave resonance parameters. File 0to500.par

$\begin{array}{lllllllllll}-1.0000 \mathrm{E}+02 & 3.8000 \mathrm{E}+01 & 1.6706 \mathrm{E}+00 & 7.7266 \mathrm{E}+01 & 4.0386 \mathrm{E}+02 & 6 & 0 & 0 & 0 & 0 & 1\end{array}$ $-4.6000 \mathrm{E}+00 \quad 3.7000 \mathrm{E}+01 \quad 9.0687 \mathrm{E}+00 \quad 2.2366 \mathrm{E}+02-6.7591 \mathrm{E}+01 \quad 0 \quad 000002$ $-2.2787 \mathrm{E}+00 \quad 3.8000 \mathrm{E}+01 \quad 6.3845 \mathrm{E}-01-2.4673 \mathrm{E}+02-4.5637 \mathrm{E}+02 \quad 0 \quad 0.008$ o 1 $\begin{array}{llllllllllllllllll}-3 & 46513-01 & 3.7000 \mathrm{E}+01 & 5.7222 \mathrm{E}-02-2.5085 \mathrm{E}+00-1.2276 \mathrm{E}+02 & 0 & 0 & 0 & 0 & 2\end{array}$ $\begin{array}{lllllllllllllll}2.77523-01 & 3.8000 \mathrm{E}+01 & 4.2587 \mathrm{E}-03 & 6.4708 \mathrm{E}+01-5.0970 \mathrm{E}+01 & 0 & 0 & 0 & 0 & 0 & 1\end{array}$

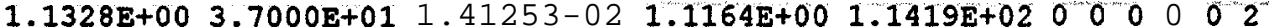

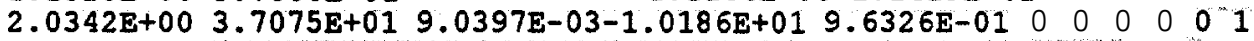
$2.7769 \mathrm{E}+00 \quad 3.7000 \mathrm{E}+01 \quad 1.0049 \mathrm{E}-03 \quad 9.8618 \mathrm{E}+01-1.3963 \mathrm{E}+01 \quad 0 \quad 000000$ $\begin{array}{llllllllllllllll}3.1392 \mathrm{E}+00 & 3.8000 \mathrm{E}+01 & 2.5018 \mathrm{E}-02-5.3397 \mathrm{E}+01 & 5.4869 \mathrm{E}+01 & 0 & 0 & 0 & 0 & 0 & 1\end{array}$ $\begin{array}{lllllllllllllllll}3.6137 \mathrm{E}+00 & 3.6387 \mathrm{E}+01 & 4.3621 \mathrm{E}-02-3.4449 \mathrm{E}+01 & 1.8262 \mathrm{E}+01 & 0 & 0 & 0 & 0 & 0 & 2\end{array}$ $4.8518 \mathrm{E}+00 \quad 3.6007 \mathrm{E}+01 \quad 5.5733 \mathrm{E}-02-1.9260 \mathrm{E}-03-4.2928 \mathrm{E}+000000002$ $5.4381 \mathrm{E}+003.7000 \mathrm{E}+01 \quad 2.7802 \mathrm{E}-02-1.7138 \mathrm{E}+02-3.7656 \mathrm{E}+02 \quad 000002$ $6.1888 \mathrm{E}+00 \quad 3.8000 \mathrm{E}+01 \quad 8.1032 \mathrm{E}-02-1.4833 \mathrm{E}+02 \quad 1.0424 \mathrm{E}+02 \quad 0 \quad 000001$

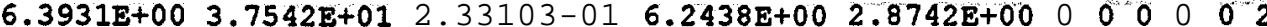

$7.0790 \mathrm{E}+003.7362 \mathrm{E}+01 \quad 1.1200 \mathrm{E}-01-7.2162 \mathrm{E}+002.5496 \mathrm{E}+0100.0160 \%$ $\begin{array}{lllllllllllllllll}7.6981 \mathrm{E}+00 & 3.8000 \mathrm{E}+01 & 2.9724 \mathrm{E}-03 & 4.1617 \mathrm{E}+01 & 1.8962 \mathrm{E}+02 & 0 & 0 & 0 & 0 & 0 & 1\end{array}$ $\begin{array}{lllllllllllll}8.7669 \mathrm{E}+00 & 3.2770 \mathrm{E}+01 & 9.3552 \mathrm{E}-01 & 4.1135 \mathrm{E}+01-5.8071 \mathrm{E}+01 & 0 & 0 & 0 & 0 & 0 & 2\end{array}$ $8.9422 \mathrm{E}+00 \quad 3.8000 \mathrm{E}+01 \quad 1.1035 \mathrm{E}-01-3.6592 \mathrm{E}+01 \quad 2.9085 \mathrm{E}+02 \quad 0 \quad 000001$

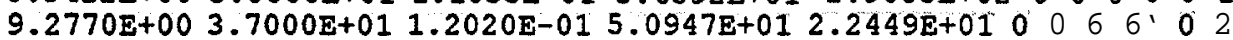
$9.7544 \mathrm{E}+003.8000 \mathrm{E}+016.60643-02 \quad 1.9849 \mathrm{E}+02-8.8398 \mathrm{E}+010000$ $1.0165 \mathrm{E}+013.7000 \mathrm{E}+01$ 5.3563E-02-4.2913E+00-6.3446E+01 0 0 o 0 o 02 $1.0707 \mathrm{E}+013.8000 \mathrm{E}+012.9322 \mathrm{E}-02-1.6389 \mathrm{E}+02-3.5148 \mathrm{E}+020000 \mathrm{0} 1 \mathrm{0}$ $1.1667 \mathrm{E}+013.7780 \mathrm{E}+015.1510 \mathrm{E}-01-5.6759 \mathrm{E}+00 \quad 8.2592 \mathrm{E}-01$ o 01000

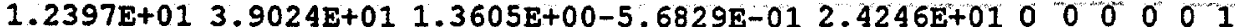

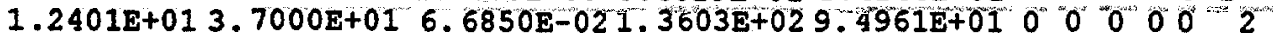

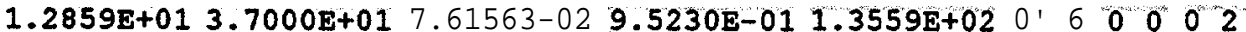
$\begin{array}{llllllllllllllll}1.3267 \mathrm{E}+01 & 3.7000 \mathrm{E}+01 & 5.9740 \mathrm{E}-02-8.8486 \mathrm{E}+01 & 9.7369 \mathrm{E}+01 & 0 & 0 & 0 & 0 & 0 & 0\end{array}$ $1.3683 \mathrm{E}+013.8000 \mathrm{E}+016.9598 \mathrm{E}-02-3.4852 \mathrm{E}+01-1.1293 \mathrm{E}+02$ o 000 o 01 1.3920E+013.8000E+01 5.7238E-01-3.6940E+02 1.1229E+02 0 o 00 o 01 $1.4112 \mathrm{E}+013.7000 \mathrm{E}+017.0656 \mathrm{E}-03-7.2601 \mathrm{E}+014.7644 \mathrm{E}+01000002$

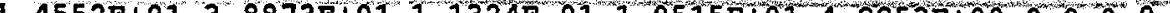

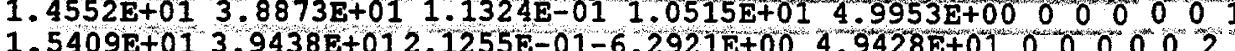
$\begin{array}{lllllllllllllllllllll}1.6087 \mathrm{E}+01 & 3.5379 \mathrm{E}+01 & 3.40143-01 & 1.3126 \mathrm{E}+01 & 9.3191 \mathrm{E}+00 & 0 & 0 & 0 & 0 & 0 & 0\end{array}$ $\begin{array}{lllllllllll}1.6642 \mathrm{E}+01 & 3.2798 \mathrm{E}+01 & 2.4477 \mathrm{E}-01 & 1.3624 \mathrm{E}+01 & 9.9609 \mathrm{E}+01 & 0 & 0 & 0 & 0 & 0 & 1\end{array}$ 1.8022E+01 3.8000E+01 2.92143-01 $6.1194 \mathrm{E}+01-5.9338 \mathrm{E}+01$ or 000 o 01 $\begin{array}{llllllllllll}1.8027 \mathrm{E}+01 & 3.7000 \mathrm{E}+01 & 9.68753-02 & 8.3183 \mathrm{E}+01-9.8024 \mathrm{E}+01 & 0 & 0 & 0 & 0 & 2\end{array}$

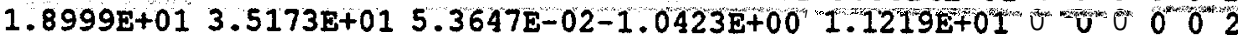

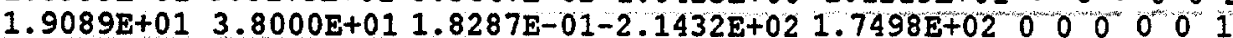
$1.9294 \mathrm{E}+013.7000 \mathrm{E}+012.7271 \mathrm{E}+00-2.9071 \mathrm{E}+013.5309 \mathrm{E}+01 \quad 0 \quad 0 \quad 0 \quad 0 \quad 02$ $2.0172 \mathrm{E}+013.8000 \mathrm{E}+017.1054 \mathrm{E}-026.0954 \mathrm{E}+01-2.6886 \mathrm{E}+01000001$

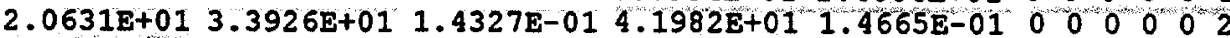
$2.1065 \mathrm{E}+013.9086 \mathrm{E}+011.3254 \mathrm{E}+001.4555 \mathrm{E}+01-1.3956 \mathrm{E}+01000.02$ $2.2931 \mathrm{E}+013.5407 \mathrm{E}+013.9623 \mathrm{E}-01-3.5853 \mathrm{E}+01$ 1.3834E+O 1 o 0000

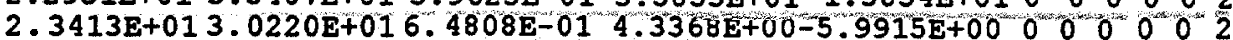
$\begin{array}{lllllllllllllllll}2.3582 \mathrm{E}+01 & 3.8000 \mathrm{E}+01 & 8.66073-01 & 1.5093 \mathrm{E}+02-4.8697 \mathrm{E}+01 & 0 & 0 & 0 & 0 & 1\end{array}$

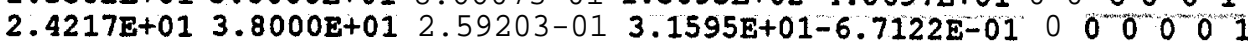
2.4349E+01 3.8000E+019.2985E-02-4.7425E+01-1.3058E+02 0 0 0 002 $2.4988 \mathrm{E}+013.7000 \mathrm{E}+019.0667 \mathrm{E}-03-1.1401 \mathrm{E}+02 \quad 9.2208 \mathrm{E}+0100010012$ $2.5527 \mathrm{E}+01 \quad 3.8000 \mathrm{E}+01 \quad 1.4672 \mathrm{E}+00-4.8947 \mathrm{E}+02 \quad 1.9611 \mathrm{E}+02 \quad 000001001$

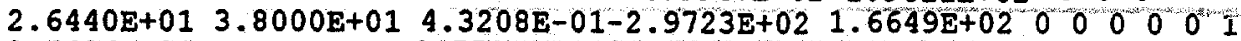
$2.6486 \mathrm{E}+013.7000 \mathrm{E}+012.7720 \mathrm{E}-01-1.0453 \mathrm{E}+02-2.0636 \mathrm{E}+01$ O 0001002 $2.7161 \mathrm{E}+013.6188 \mathrm{E}+015.3460 \mathrm{E}-02-1.1117 \mathrm{E}+00-3.8587 \mathrm{E}+01000001$ $2.7783 \mathrm{E}+013.7000 \mathrm{E}+016.0174 \mathrm{E}-01-8.0364 \mathrm{E}+01-6.7009 \mathrm{E}+00000002$

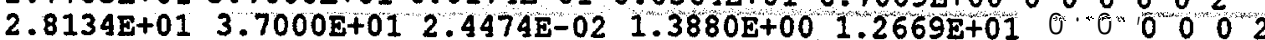
$\begin{array}{llllllllllllllll}2.8335 \mathrm{E}+01 & 3.8000 \mathrm{E}+01 & 2.0237 \mathrm{E}-01-2.4568 \mathrm{E}+01 & 8.0230 \mathrm{E}+01 & 0 & 0 & 0 & 0 & 0 & 1\end{array}$ $\begin{array}{llllllllllllllllllll}2.8733 \mathrm{E}+01 & 3.7000 \mathrm{E}+01 & 2.2120 \mathrm{E}-02 & 6.0583 \mathrm{E}-02 & 6.0711 \mathrm{E}+01 & 0 & 0 & 0 & 0 & 0 & 2.07\end{array}$ $2.9642 \mathrm{E}+013.2121 \mathrm{E}+01$ 1.4628E-01-7.5304E+00 1.3753E+01 0 O 01002 $3.0591 \mathrm{E}+013.8000 \mathrm{E}+012.3526 \mathrm{E}-01-1.5694 \mathrm{E}+018.6071 \mathrm{E}+010000001$ $3.0866 \mathrm{E}+013.6292 \mathrm{E}+01$ 4.7442E-01-6.1763E-01 $1.7764 \mathrm{E}+01$ o 00002 $3.2025 \mathrm{E}+013.7996 \mathrm{E}+01$ 5.3019E-01-1.9502E+01 7.6605E+01 0 o 0001 
3.2069E+01 3.7000E+01 1.2675E+00-1.6137E+00 $4.9449 E+01$ o 00 o 000

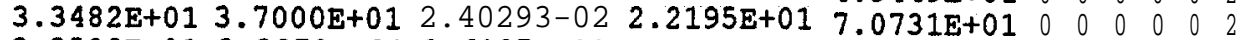

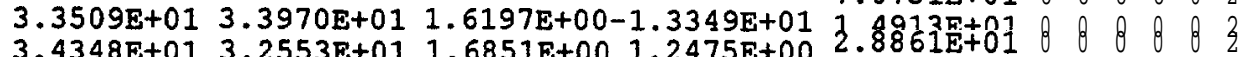
$3.4348 \mathrm{E}+013.2553 \mathrm{E}+01 \quad 1.6851 \mathrm{E}+001.2475 \mathrm{E}+00$

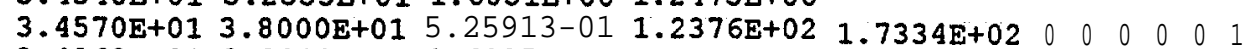
$\begin{array}{llllllllll}3.4869 \mathrm{E}+01 & 3.8000 \mathrm{E}+01 & 1.6027 \mathrm{E}+00-2.0268 \mathrm{E}+01 & 9.8151 \mathrm{E}+01 & 0 & 0 & 0 & 0 & 0 & 1\end{array}$

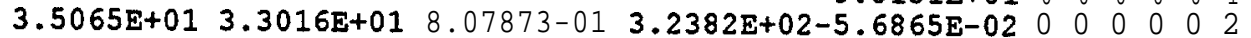

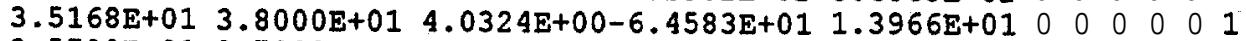

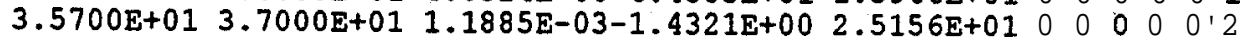

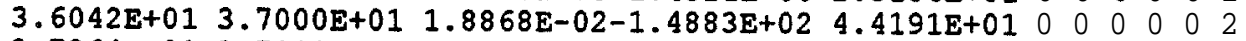
$\begin{array}{lllllllllll}3.7264 \mathrm{E}+01 & 3.7000 \mathrm{E}+01 & 3.5617 \mathrm{E}-03 & 1.8070 \mathrm{E}+02-1.9880 \mathrm{E}+01 & 0 & 0 & 0 & 0 & 0 & 2\end{array}$ $\begin{array}{llllllllll}3.8335 \mathrm{E}+01 & 3.7000 \mathrm{E}+01 & 3.0332 \mathrm{E}-01-2.2308 \mathrm{E}+02-5.8570 \mathrm{E}+01 & 0 & 0 & 0 & 0 & 0 & 2\end{array}$

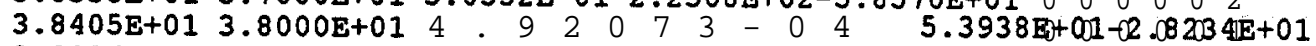

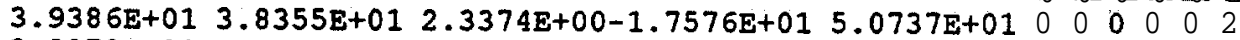

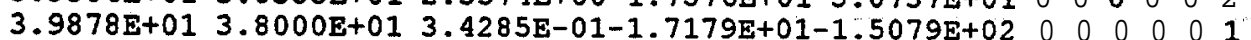

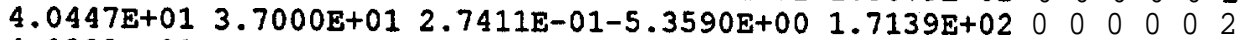

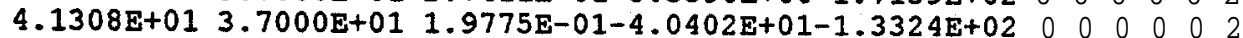

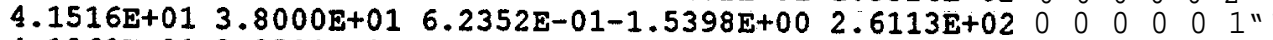

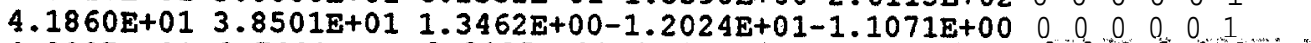
$4.2207 \mathrm{E}+013.7000 \mathrm{E}+012.8135 \mathrm{E}-01-6.0092 \mathrm{E}+01-1.5300 \mathrm{E}+01$ 0 010000 $\begin{array}{llllllllll}4.2696 \mathrm{E}+01 & 3.8000 \mathrm{E}+01 & 2.4092 \mathrm{E}-01 & 5.3969 \mathrm{E}+00-1.2704 \mathrm{E}-01 & 0 & 0 & 0 & 0 & 0 & 2\end{array}$

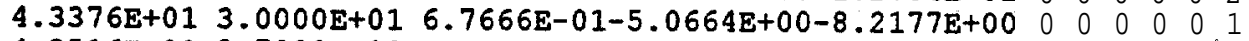

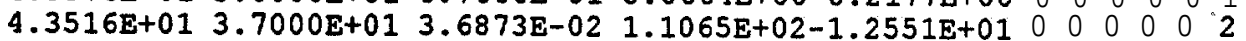

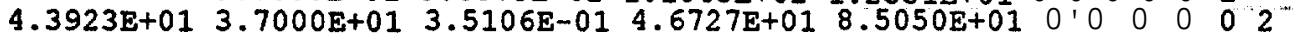
$\begin{array}{llllllllll}4.4575 \mathrm{E}+01 & 3.7000 \mathrm{E}+01 & 6.2916 \mathrm{E}-01-8.6765 \mathrm{E}+01 & 4.2343 \mathrm{E}+01 & 0 & 0 & 0 & 0 & 0 & 2^{\prime}\end{array}$

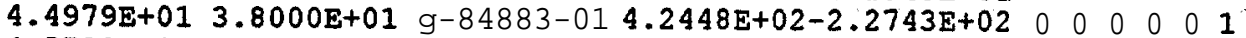

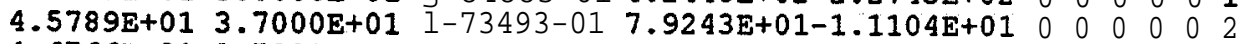
$\begin{array}{lllllllll}4.6766 \mathrm{E}+01 & 3.7000 \mathrm{E}+01 & 7.4151 \mathrm{E}-01-6.8319 \mathrm{E}+01-4.8356 \mathrm{E}+01 & 0 & 0 & 0 & 0 & 0 & 2\end{array}$ 4.7014E+01 3.7000E+01 7.0046E-01-1.4848E-02 8.4393E+01 00000001 $\begin{array}{llllllllllll}4.7939 \mathrm{E}+01 & 3.3785 \mathrm{E}+01 & 7.1017 \mathrm{E}-01 & 1.9405 \mathrm{E}+01 & 2.7217 \mathrm{E}+01 & 0 & 0 & 0 & \infty & 0\end{array}$ $\begin{array}{llllllllll}4.8324 \mathrm{E}+01 & 3.8000 \mathrm{E}+01 & 1.0793 \mathrm{E}+00 & 1.7324 \mathrm{E}+02-2.3674 \mathrm{E}+01 & 0 & 0 & 0 & 0 & 0 & 1\end{array}$ $4.8760 \mathrm{E}+013.8000 \mathrm{E}+01 \quad 8.7660 \mathrm{E}-01 \quad 9.6290 \mathrm{E}+00-5.6104 \mathrm{E}+010000001$ $4.9431 \mathrm{E}+013.6223 \mathrm{E}+01$ 8.7001E-01-5.4629E+00-6.1861E+00 0 O 0 0 002 $5.0079 \mathrm{E}+013.4367 \mathrm{E}+012.8087 \mathrm{E}-01 \quad 8.7051 \mathrm{E}+000-1.5494 \mathrm{E} 00 \mathrm{D} 0001$ $5.0447 \mathrm{E}+013.3325 \mathrm{E}+019.9568 \mathrm{E}-01-3.5105 \mathrm{E}+01-1.2034 \mathrm{E}-010000001$

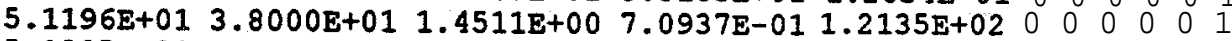

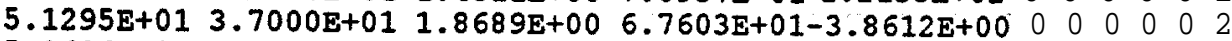

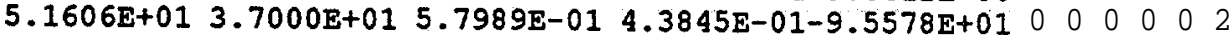

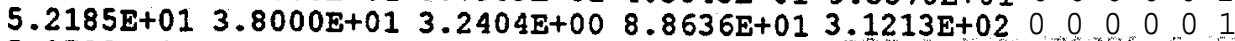
$5.2700 \mathrm{E}+013.7000 \mathrm{E}+01 \quad 5.1204 \mathrm{E}-05 \quad 3.2229 \mathrm{E}+02 \quad 4.4676 \mathrm{E}+01 \quad 0^{\circ} 0^{4} 00^{\circ} 0^{\prime \prime 2}$ $\begin{array}{llllllllll}5.2774 \mathrm{E}+01 & 3.7000 \mathrm{E}+01 & 2.6903 \mathrm{E}-03-1.5056 \mathrm{E}+02-6.6018 \mathrm{E}+01 & 0 & 0 & 0 & 0 & 0 & 2\end{array}$ $5.3403 \mathrm{E}+013.8000 \mathrm{E}+015.8328 \mathrm{E}-01-8.0729 \mathrm{E}+01 \quad 1.5036 \mathrm{E}+01 \quad 0 \quad 000$ $5.4177 \mathrm{E}+013.7000 \mathrm{E}+01$ 1.0946E-01-6.3420E+01-2.1262E+01 010000001

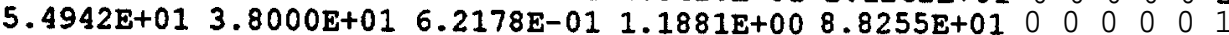

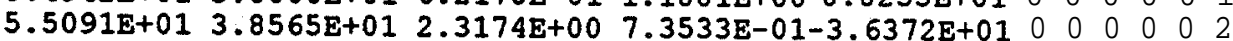

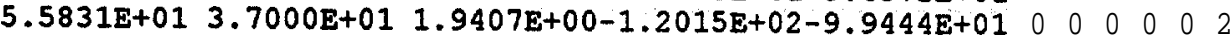

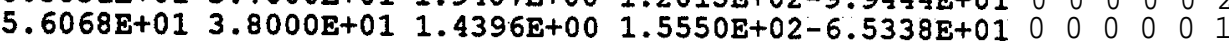

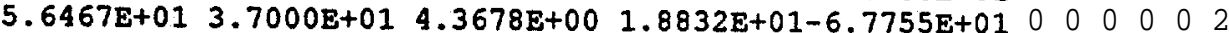

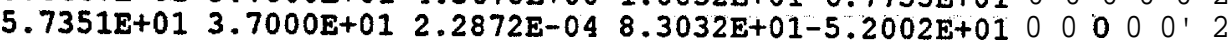

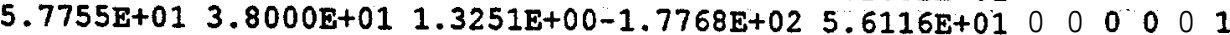

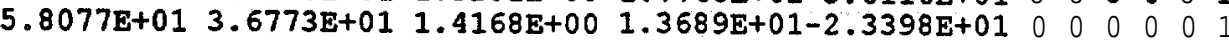

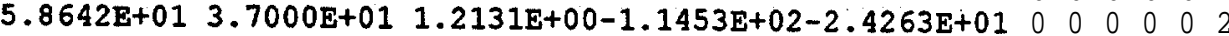

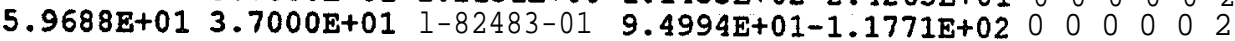

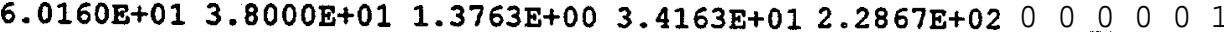

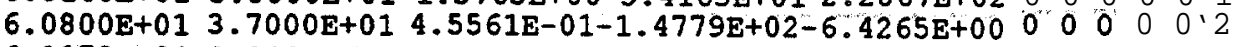

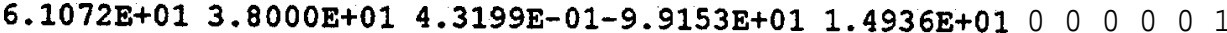

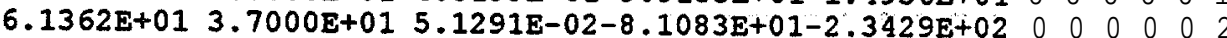

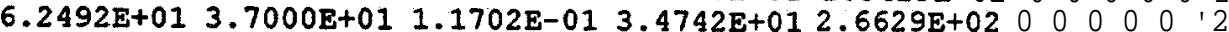

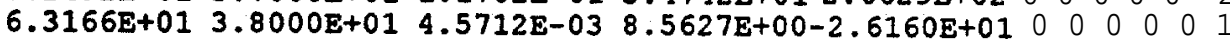

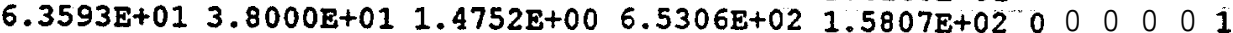

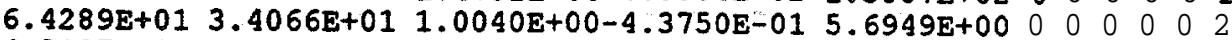
$6.5197 \mathrm{E}+013.7000 \mathrm{E}+01 \quad 2.4057 \mathrm{E}-05 \quad 1.6653 \mathrm{E}+01-1.5138 \mathrm{E}+01 \quad 0-0-002$.

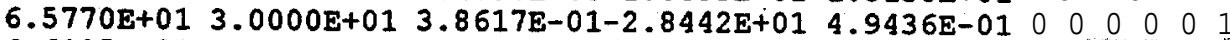

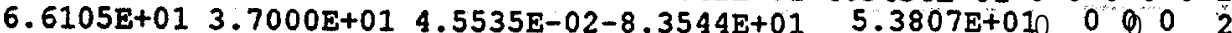
$6.7199 \mathrm{E}+013.7001 \mathrm{E}+01 \quad 6.8204 \mathrm{E}-02$

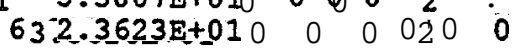

$5.4743 \mathrm{E}+01000000$ 
$6.7578 \mathrm{E}+013.7000 \mathrm{E}+011.9866 \mathrm{E}-05-4.5006 \mathrm{E}+01-5.2927 \mathrm{E}+0100000012$ $6.8371 \mathrm{E}+013.7000 \mathrm{E}+012.8851 \mathrm{E}-02-1.8627 \mathrm{E}-01-3.8908 \mathrm{E}+0100000012$ $\begin{array}{lllllllllllllllll}6.8580 \mathrm{E}+01 & 3.8000 \mathrm{E}+01 & 1.2451 \mathrm{E}-01 & 6.8655 \mathrm{E}+01-5.4703 \mathrm{E}+01 & 0 & 0 & 0 & 0 & 0 & 1\end{array}$ $6.9204 \mathrm{E}+013.7000 \mathrm{E}+015.7530 \mathrm{E}-01-8.7337 \mathrm{E}+01-1.0245 \mathrm{E}+0200000012$ $7.0260 \mathrm{E}+013.8000 \mathrm{E}+013.9596 \mathrm{E}-01-1.8081 \mathrm{E}+02-2.3799 \mathrm{E}+02 \quad 0 \quad 0 \quad 000001$ $7.0374 \mathrm{E}+013.8000 \mathrm{E}+01$ 1.4692 $\mathrm{E}+002.1952 \mathrm{E}+02-6.8254 \mathrm{E}-0300000001$ $7.0451 \mathrm{E}+013.7000 \mathrm{E}+01 \quad 1.4375 \mathrm{E}+00 \quad 8.1521 \mathrm{E}+013.4528 \mathrm{E}+0200000002$

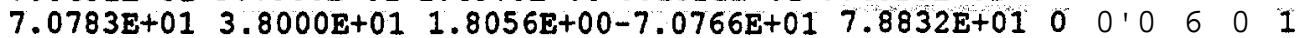
$7.1652 \mathrm{E}+013.7000 \mathrm{E}+012.5206 \mathrm{E}-01-1.7983 \mathrm{E}+02-5.7181 \mathrm{E}-01000002$ $7.2363 \mathrm{E}+013.7000 \mathrm{E}+012.5078 \mathrm{E}+00-9.0887 \mathrm{E}+01-1.4277 \mathrm{E}+0100000002$ $7.2888 \mathrm{E}+01 \quad 3.8000 \mathrm{E}+012.5330 \mathrm{E}-01-3.7380 \mathrm{E}-02 \quad 2.7583 \mathrm{E}+02$ o 00 o 0001

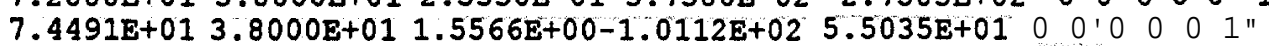

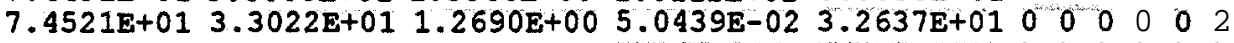

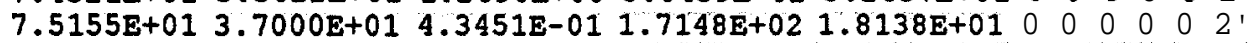
$\begin{array}{lllllllllllllllll}7.5552 \mathrm{E}+01 & 3.8000 \mathrm{E}+01 & 1.7836 \mathrm{E}+00 & 2.4946 \mathrm{E}+02 & 1.4978 \mathrm{E}+01 & 0 & 0 & 0 & 0 & 0 & 1\end{array}$

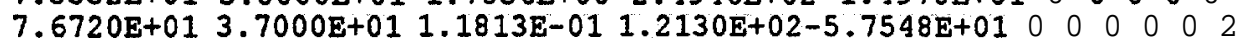
$\begin{array}{llllllllll}7.7073 \mathrm{E}+01 & 3.7000 \mathrm{E}+01 & 1.0119 \mathrm{E}-04 & 6.6530 \mathrm{E}+01 & 2.4448 \mathrm{E}+01 & 0 & 0 & 0 & 0 & 0\end{array}$ $7.7481 \mathrm{E}+013.7000 \mathrm{E}+01 \quad 7.7208 \mathrm{E}-01-1.3193 \mathrm{E}+01 \quad 1.1859 \mathrm{E}+02$ o 010002 $\begin{array}{llllllllllllllll}7.8068 \mathrm{E}+01 & 3.8000 \mathrm{E}+01 & 1.2270 \mathrm{E}+00 & 2.1190 \mathrm{E}+00 & 9.2980 \mathrm{E}+01 & 0 & 0 & 0 & 0 & 0 & 1\end{array}$ $7.8418 \mathrm{E}+013.6729 \mathrm{E}+017.4817 \mathrm{E}-023.3425 \mathrm{E}+013.7541 \mathrm{E}+01 \cdots 000000$

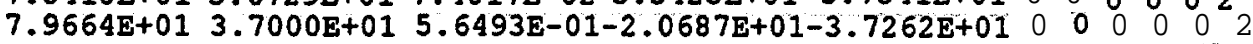

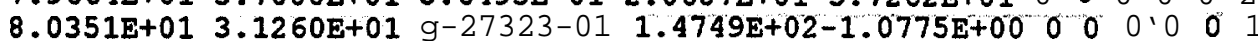
$8.0791 \mathrm{E}+013.4970 \mathrm{E}+01$ 1.0793E-01-3.7157E+01-1.4373E-02 0 o 0 o 002 $8.0808 \mathrm{E}+013.3650 \mathrm{E}+01$ 1.1360E-04 $4.3976 \mathrm{E}+01-2.0122 \mathrm{E}+02 \quad 0 \quad 0 \quad 000$

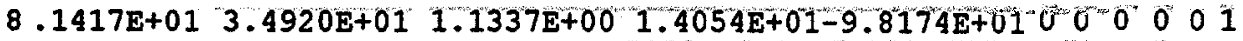

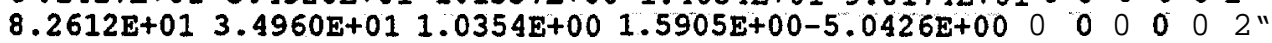

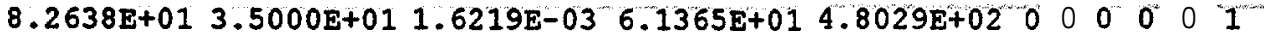

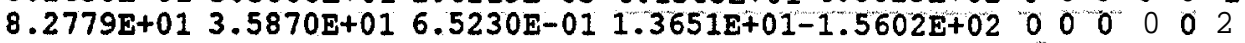

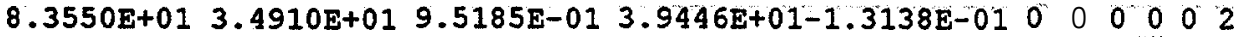
$8.3846 \mathrm{E}+013.4930 \mathrm{E}+018.0790 \mathrm{E}-01$ 1.6432E+02-1.0284E+02 01000001 $8.4028 \mathrm{E}+013.4940 \mathrm{E}+012.2449 \mathrm{E}+00 \quad 1.9623 \mathrm{E}+021.2372 \mathrm{E}+0200010002$ $8.4314 \mathrm{E}+013.4680 \mathrm{E}+01 \quad 8.1583 \mathrm{E}-01 \quad 6.3633 \mathrm{E}-01-6.9512 \mathrm{E}+01 \quad 0000001$

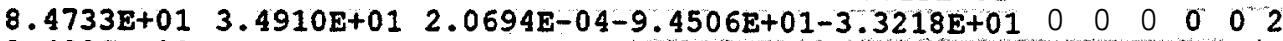
$8.4991 \mathrm{E}+01 \quad 3.4970 \mathrm{E}+01 \quad 1.8157 \mathrm{E}+00 \quad 1.1856 \mathrm{E}+02 \quad 3.6962 \mathrm{E}+02 \quad 000000$ 8.5711E+01 3.4960E+01 5.7108E-01-3.2891E+02-1.5309E+02 0000002

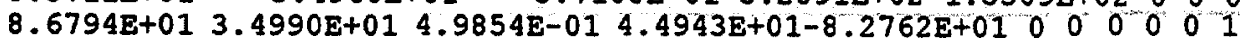

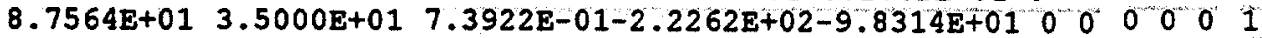
$8.8670 \mathrm{E}+013.4500 \mathrm{E}+012.8136 \mathrm{E}+00-1.4749 \mathrm{E}+02 \quad 3.0505 \mathrm{E}+02000001$

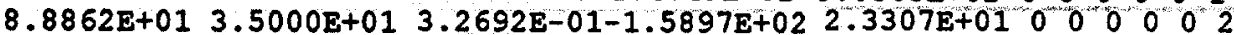
$8.9099 \mathrm{E}+013.5010 \mathrm{E}+011.6267 \mathrm{E}-01-6.9350 \mathrm{E}+00 \quad 9.1877 \mathrm{E}-01 \quad 0 \quad 0 \quad 0 \quad 0 \quad 0 \quad 2$ 8.9774E+01 3.4990E+01 6.0662E-01-4.7702E+01-6.4154E+01 0 U 0002 $9.0343 \mathrm{E}+013.4950 \mathrm{E}+013.9393 \mathrm{E}+00-1.2650 \mathrm{E}+024.1948 \mathrm{E}+01 \mathrm{O} 0 \mathrm{O} 0 \mathrm{O} 02$

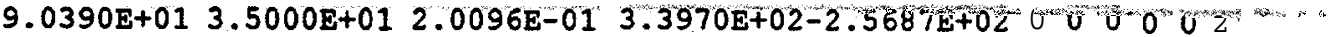

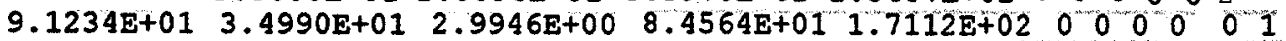
$9.2004 \mathrm{E}+013.4990 \mathrm{E}+015.1366 \mathrm{E}-01-4.6513 \mathrm{E}-01-6.4345 \mathrm{E}+01000010$ $9.2563 \mathrm{E}+01 \quad 3.4990 \mathrm{E}+012.1016 \mathrm{E}+00 \quad 8.0586 \mathrm{E}-013.1840 \mathrm{E}+01000000$ $9.3123 \mathrm{E}+013.5000 \mathrm{E}+01$ 9.5128E-02 1. $6031 \mathrm{E}+027.9611 \mathrm{E}+01$ o 000012 $9.3273 \mathrm{E}+013.5000 \mathrm{E}+012.6732 \mathrm{E}-015.9854 \mathrm{E}+00-8.7625 \mathrm{E}+01$ o 00001

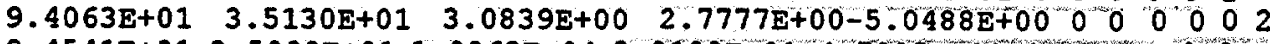

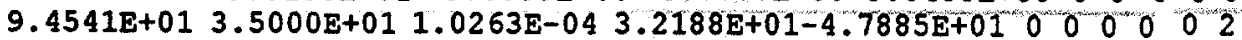

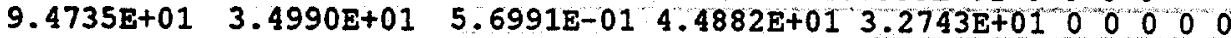
$9.5447 \mathrm{E}+013.4990 \mathrm{E}+011.1160 \mathrm{E}+00-2.3760 \mathrm{E}+02-3.1271 \mathrm{E}+02$ o 0 o 0102

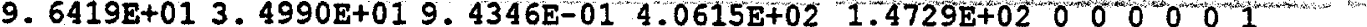
$9.6666 \mathrm{E}+013.5000 \mathrm{E}+013.5060 \mathrm{E}-02 \quad 1.5882 \mathrm{E}+02-2.9522 \mathrm{E}+01000002$ $9.8066 \mathrm{E}+01 \quad 3.4930 \mathrm{E}+01 \quad 2.9047 \mathrm{E}+00 \quad 1.8410 \mathrm{E}+02-1.7067 \mathrm{E}-01 \quad 00000$

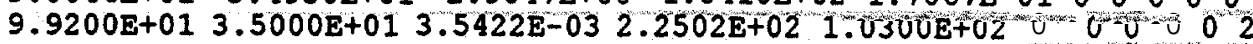
$9.9428 \mathrm{E}+013.4980 \mathrm{E}+014.8651 \mathrm{E}-011.0402 \mathrm{E}+011.3793 \mathrm{E}+02000001$ $1.0013 \mathrm{E}+023.5000 \mathrm{E}+012.2640 \mathrm{E}-041.4029 \mathrm{E}+02 \quad 2.4324 \mathrm{E}+02 \quad 000202$

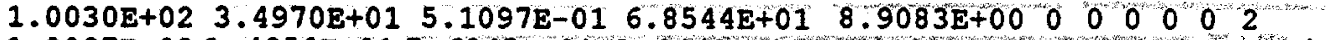
$1.0097 \mathrm{E}+023.4950 \mathrm{E}+017.6245 \mathrm{E}-01-3.0442 \mathrm{E}+01-1.4315 \mathrm{E}+00$ o 00002 $\begin{array}{lllllllllllllllllll}1.0115 \mathrm{E}+02 & 3.5000 \mathrm{E}+01 & 9.0454 \mathrm{E}-04-2.3032 \mathrm{E}+02 & 9.0240 \mathrm{E}+010 & 0 & 0 & 0 & 0 & 1\end{array}$ $1.0180 \mathrm{E}+023.4950 \mathrm{E}+013.9713 \mathrm{E}-01-4.4247 \mathrm{E}+013.1565 \mathrm{E}+010000.01$ $\begin{array}{llllll}1.0213 \mathrm{E}+02 & 3.5000 \mathrm{E}+01 & 2.0173 \mathrm{E}-05-4.6398 \mathrm{E}+02 & 3.3911 \mathrm{E}+0 \mathrm{y} 2 & 0 & 0\end{array}$

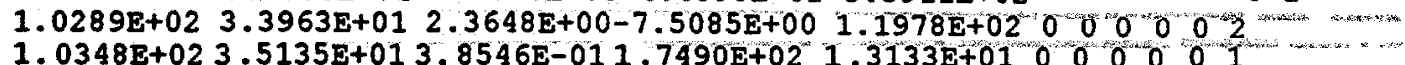


$\begin{array}{lllllllllll}1.0352 \mathrm{E}+02 & 4.0367 \mathrm{E}+01 & 1.1731 \mathrm{E}+00 & 1.7712 \mathrm{E}+01 & 5.2837 \mathrm{E}+00 & 0 & 0 & 0 & 0 & 0 & 2\end{array}$

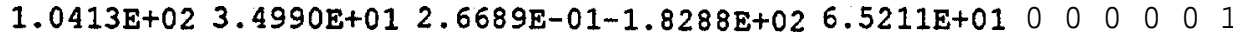

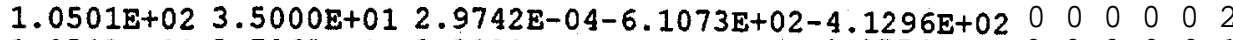

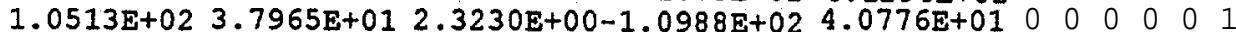

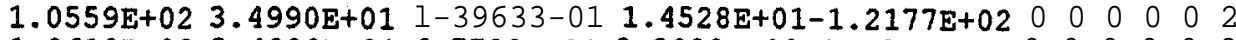

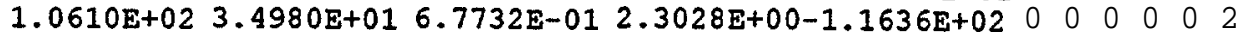
$\begin{array}{lllllllllll}1.0680 \mathrm{E}+02 & 3.4990 \mathrm{E}+01 & 5.76113-01 & 3.5299 \mathrm{E}+02-3.0501 \mathrm{E}+02 & 0 & 0 & 0 & 0 & 0 & 1\end{array}$

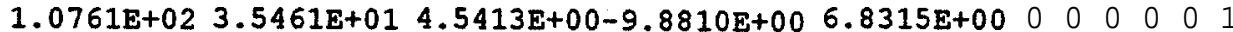

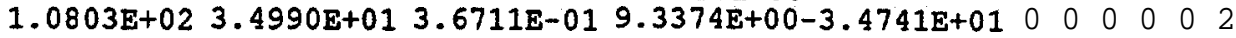

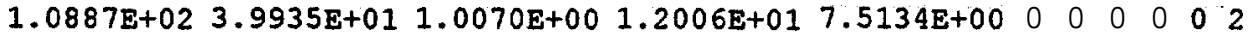

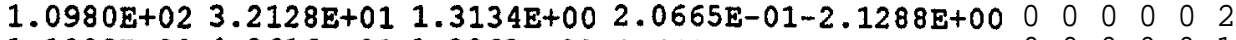

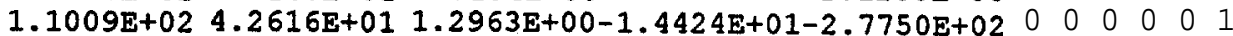

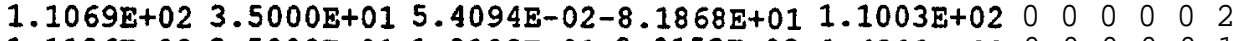

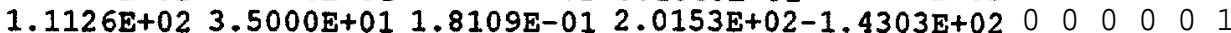
$\begin{array}{lllllllllll}1.1163 \mathrm{E}+02 & 3.9806 \mathrm{E}+01 & \mathrm{~g}-55363-01 & 5.4421 \mathrm{E}+00 & 3.3274 \mathrm{E}+01 & 0 & 0-0 & 0 & 6 & -2\end{array}$

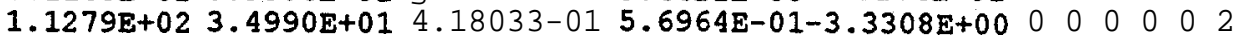
$1.1348 \mathrm{E}+02 \quad 3.7708 \mathrm{E}+01 \quad 1.7081 \mathrm{E}+00-1.0415 \mathrm{E}+02-1.0970 \mathrm{E}+02000000001$

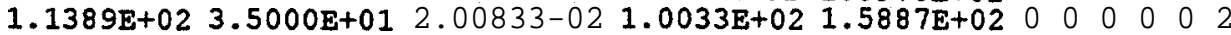

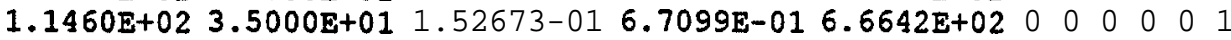

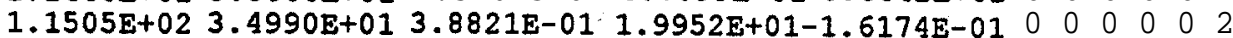

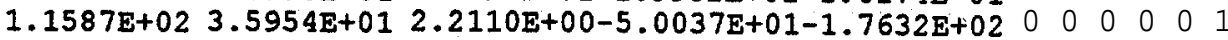
$1.1600 \mathrm{E}+024.0735 \mathrm{E}+011.0425 \mathrm{E}+00-1.5288 \mathrm{E}+021.7693 \mathrm{E}+02000001002$ $1.1670 \mathrm{E}+023.5000 \mathrm{E}+01$ 1.7460E-05-9.0360E+01-9.1900E+01 00000003 $1.1780 \mathrm{E}+02 \quad 3.5000 \mathrm{E}+01 \quad 3.3326 \mathrm{E}-01-4.7527 \mathrm{E}+02-2.1047 \mathrm{E}+02 \quad 00000001$

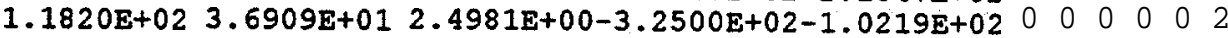

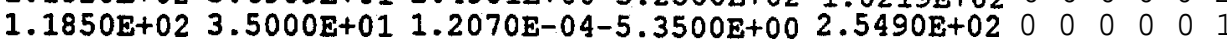

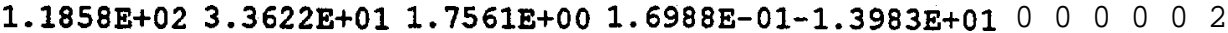

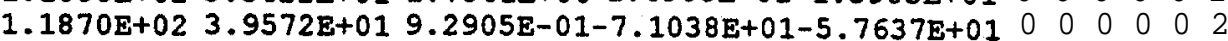

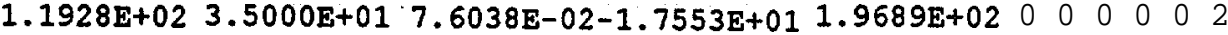

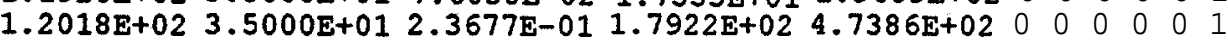

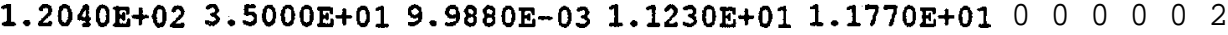

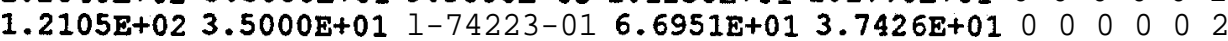

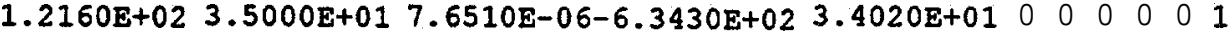

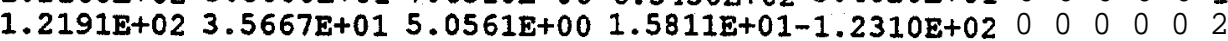

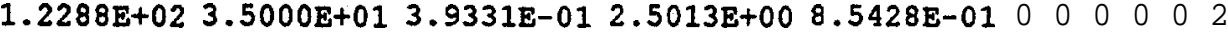
$1.2351 \mathrm{E}+02 \quad 3.5000 \mathrm{E}+01 \quad 5.6226 \mathrm{E}-01 \quad 1.2787 \mathrm{E}+021.0008 \mathrm{E}+020000001$ $\begin{array}{llllllllllllll}1.2379 \mathrm{E}+02 & 3.5000 \mathrm{E}+01 & 1.9635 \mathrm{E}-01-2.0984 \mathrm{E}+02 & 4.4712 \mathrm{E}+01 & 0 & 0 & 0 & 0 & 0 & 1\end{array}$

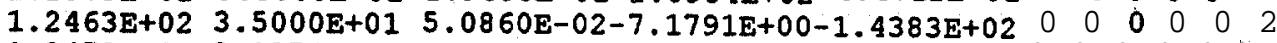

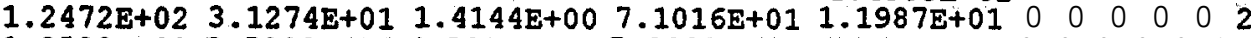

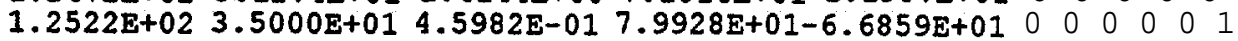

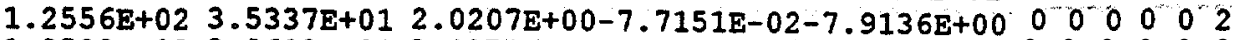
$1.2588 \mathrm{E}+02 \quad 3.9618 \mathrm{E}+013.4974 \mathrm{E}+00-6.3398 \mathrm{E}+01-2.4272 \mathrm{E}+020000002$

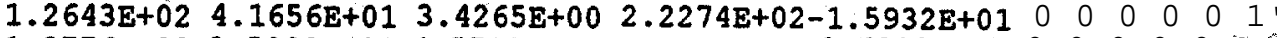

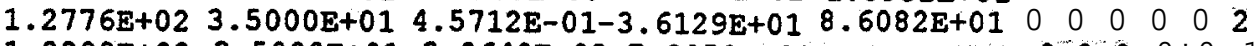

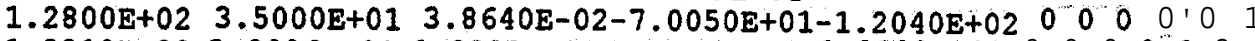
$1.2810 \mathrm{E}+023.9926 \mathrm{E}+01 \quad 1.2822 \mathrm{E}+00-1.0554 \mathrm{E}+011.2102 \mathrm{E}+020000002$ 1.2950E+02 3.5000E+01 7.80203-01 2.4770E+01 9.0670E+01 00000001

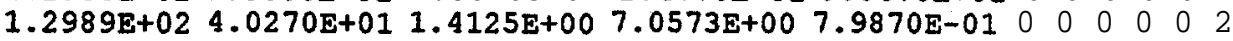

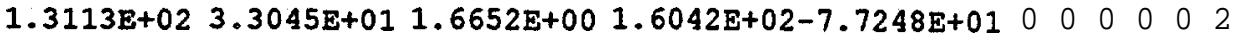

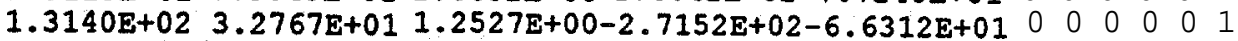

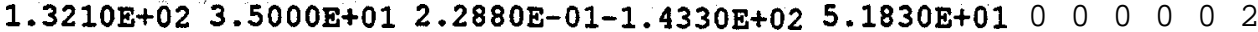

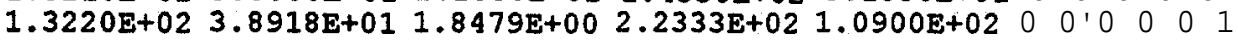

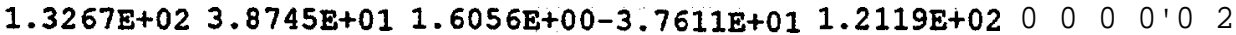
$1.3310 \mathrm{E}+02 \quad 3.5000 \mathrm{E}+01 \quad 7.0270 \mathrm{E}-01-8.6900 \mathrm{E}+01-7.9810 \mathrm{E}+01 \quad 0000001$

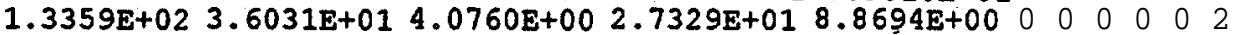

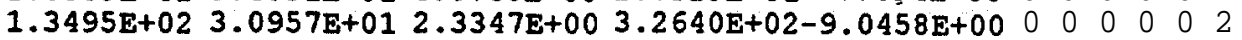

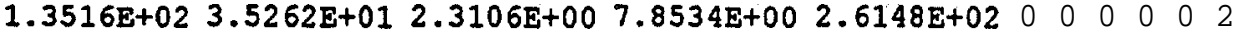
$\begin{array}{llllllllllllllll}1.3547 \mathrm{E}+02 & 3.0222 \mathrm{E}+01 & 3.2148 \mathrm{E}+00 & 1.4478 \mathrm{E}+01 & 2.5137 \mathrm{E}+02 & 0 & 0 & 0 & 0 & 0 & 1\end{array}$

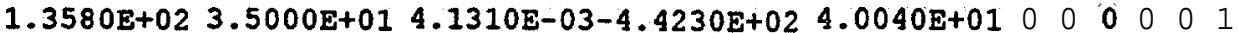
$\begin{array}{lllllllllllllllll}1.3626 \mathrm{E}+02 & 4.6985 \mathrm{E}+01 & 2.8595 \mathrm{E}+00-5.5433 \mathrm{E}+00 & 1.3540 \mathrm{E}+01 & 0 & 0 & 0 & 0 & 0 & 2\end{array}$

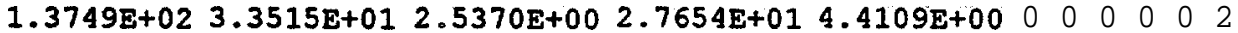
$\begin{array}{lllllllllllllllll}1.3850 \mathrm{E}+02 & 3.5000 \mathrm{E}+01 & 1-95503-02 & 5.2450 \mathrm{E}+02 & 3.3890 \mathrm{E}+02 & 0 & 0 & 0 & 0 & 0 & 2 & \cdots\end{array}$ $\begin{array}{lllllllllll}1.3910 \mathrm{E}+02 & 3.5000 \mathrm{E}+01 & 5.79103-01 & 1.8120 \mathrm{E}+01 & 2.7350 \mathrm{E}+01 & 0 & 0 & 0 & 0 & 0 & 1\end{array}$

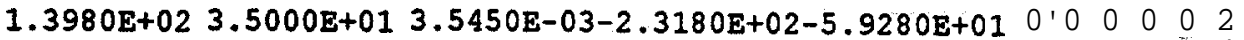

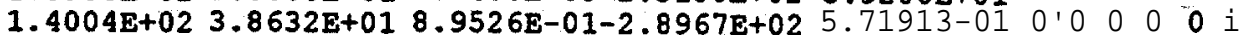
$1.4030 \mathrm{E}+023.5000 \mathrm{E}+013.80903-01 \quad 5.1900 \mathrm{E}+007.5580 \mathrm{E}+01000000$ 


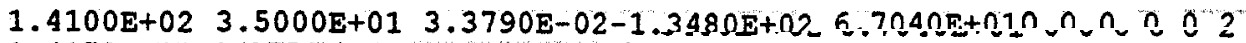
$1.4178 \mathrm{E}+02 \quad 4.8758 \mathrm{E}+012.0905 \mathrm{E}+00 \quad 4.5343 \mathrm{E}+01-8.4717 \mathrm{E}+010.000001$ $\begin{array}{lllllllllllll}1.4210 \mathrm{E}+02 & 3.4360 \mathrm{E}+01 & 3.2086 \mathrm{E}+00 & 3.0974 \mathrm{E}+01 & 6.2168 \mathrm{E}+00 & 0 & 0 & 0 & 0 & 2\end{array}$ $1.4249 \mathrm{E}+023.5000 \mathrm{E}+01 \quad 6.2180 \mathrm{E}-01-2.7306 \mathrm{E}+02$ 4.7907 $\mathrm{E}+02000001$ $1.4277 \mathrm{E}+02 \quad 3.5000 \mathrm{E}+01 \quad 4.1227 \mathrm{E}-03 \quad 2.0385 \mathrm{E}+02-3.6239 \mathrm{E}+02 \quad 0 \quad 0-0 \quad 6 \quad 02$ $\begin{array}{llllllllllll}1.4383 \mathrm{E}+02 & 3.5000 \mathrm{E}+01 & 1.5627 \mathrm{E}-02 & 6.3733 \mathrm{E}+02-8.8379 \mathrm{E}+01 & 0 & 0 & 0 & 0 & 0 & 1\end{array}$ $1.4438 \mathrm{E}+023.5000 \mathrm{E}+011.1688 \mathrm{E}-01-3.2454 \mathrm{E}+02-1.8784 \mathrm{E}+0200002$ $\begin{array}{lllllllll}1.4553 \mathrm{E}+02 & 3.9010 \mathrm{E}+01 & 4.3835 \mathrm{E}+00 & 1.1188 \mathrm{E}+01-6.6269 \mathrm{E}+01 & 0 & 0 & 0 & 0 & 0\end{array}$ $\begin{array}{lllllllllll}1.4575 \mathrm{E}+02 & 4.0978 \mathrm{E}+01 & 1.7675 \mathrm{E}+00 & 3.4684 \mathrm{E}+02-1.7532 \mathrm{E}-01 & 0 & 0 & 0 & 6 & 0 & 2\end{array}$ $1.4588 \mathrm{E}+023.5000 \mathrm{E}+012.4691 \mathrm{E}-03-3.0822 \mathrm{E}+02-1.4694 \mathrm{E}+000^{\prime} 6^{\prime} 6002$ $\begin{array}{lllllllll}1.4697 \mathrm{E}+02 & 3.5000 \mathrm{E}+01 & 6.4329 \mathrm{E}-03 & 3.3465 \mathrm{E}+02-1.5992 \mathrm{E}+02 & 0 & 0-0 & 0 & 0 & 2\end{array}$

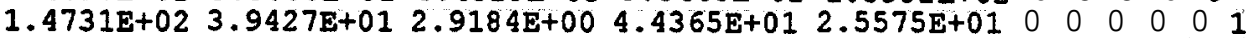
$1.4780 \mathrm{E}+02 \quad 3.5000 \mathrm{E}+01 \quad 2.7180 \mathrm{E}-03 \quad 4.5320 \mathrm{E}+01-1.4630 \mathrm{E}+02 \quad 0 \quad 000001$ $1.4873 \mathrm{E}+023.5000 \mathrm{E}+01 \quad 5.0062 \mathrm{E}-02 \quad 3.2797 \mathrm{E}+011.1876 \mathrm{E}+0200002$

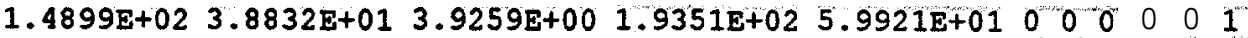
$1.4930 \mathrm{E}+02 \quad 3.5010 \mathrm{E}+01 \quad 3.9331 \mathrm{E}-01-3.1138 \mathrm{E}+01 \quad 2.0174 \mathrm{E}-01-0=0.001$ $1.4993 \mathrm{E}+024.4042 \mathrm{E}+01$ 1.6196E+00-1.7333E+02-1.8660E+01 0001602 $1.5041 \mathrm{E}+023.5942 \mathrm{E}+013.2857 \mathrm{E}-01 \quad 2.7657 \mathrm{E}+00-3.7099 \mathrm{E}+01000002$ $1.5098 \mathrm{E}+023.6047 \mathrm{E}+011.12263-015.5424 \mathrm{E}+01-1.2906 \mathrm{E}+01000002$

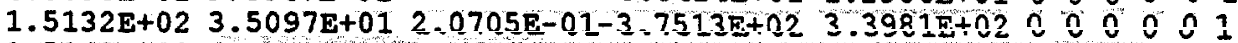
$1.5163 \mathrm{E}+023.5835 \mathrm{E}+017.3989 \mathrm{E}-01-2.2337 \mathrm{E}+00-4.4869 \mathrm{E}+0000002$ $\begin{array}{lllllllll}1.5251 \mathrm{E}+02 & 3.5000 \mathrm{E}+01 & 3.0431 \mathrm{E}-02-9.3293 \mathrm{E}+01 & 3.6939 \mathrm{E}+01 & 0 & 0 & 0 & 0 & 0\end{array}$ $1.5251 \mathrm{E}+023.5000 \mathrm{E}+01 \quad 4.1600 \mathrm{E}-02 \quad 7.0177 \mathrm{E}+01-1.4623 \mathrm{E}+02000002$ $1.5337 \mathrm{E}+02 \quad 3.6896 \mathrm{E}+013.9866 \mathrm{E}+002.0817 \mathrm{E}+01 \quad 5.2935 \mathrm{E}+0100000$ $1.5410 \mathrm{E}+023.5000 \mathrm{E}+01 \quad 5.0968 \mathrm{E}-02-7.1077 \mathrm{E}+00-2.2758 \mathrm{E}+02000001$ $1.5430 \mathrm{E}+02 \quad 3.5000 \mathrm{E}+012.1096 \mathrm{E}-023.8558 \mathrm{E}+012.8105 \mathrm{E}+0000000$ $1.5477 \mathrm{E}+02 \quad 3.7049 \mathrm{E}+01 \quad 1.0903 \mathrm{E} 0=2-27.225+013.9803 \mathrm{E}+0100002$ $\begin{array}{lllllllllll}1.5527 \mathrm{E}+02 & 3.7355 \mathrm{E}+01 & 8.3358 \mathrm{E}-01-1.5521 \mathrm{E}+02 & 1.1507 \mathrm{E}+02 & 0 & 0 & 0 & 0 & 0 & 1\end{array}$

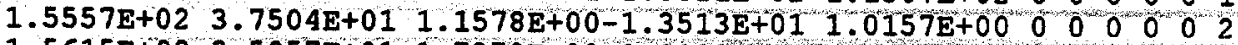
$1.5615 \mathrm{E}+023.5057 \mathrm{E}+01 \quad 1.7278 \mathrm{E}+001.1123 \mathrm{E}+01 \quad 8.5584 \mathrm{E}+000000001$ $1.5678 \mathrm{E}+02 \quad 3.5368 \mathrm{E}+013.1553 \mathrm{E}+00 \quad 1.0545 \mathrm{E}+01-2.6859 \mathrm{E}+01000002$ $1.5743 \mathrm{E}+023.6750 \mathrm{E}+01 \quad 8.4588 \mathrm{E}-01 \quad 1.8298 \mathrm{E}+01 \quad 1.2134 \mathrm{E}+000000001$

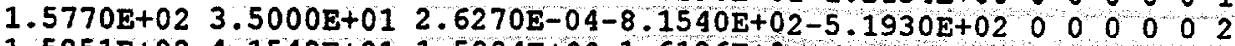
$\begin{array}{lllllllllll}1.5851 \mathrm{E}+02 & 4.1542 \mathrm{E}+01 & 1.5284 \mathrm{E}+00 & 1.6186 \mathrm{E}+02 & 8.7832 \mathrm{E}+01 & 0 & 0 & 0 & 0 & 0 & 1\end{array}$ $1.5860 \mathrm{E}+023.5000 \mathrm{E}+01$ 1.5075E-02-7.1123E+01-4.7946E+01 000002

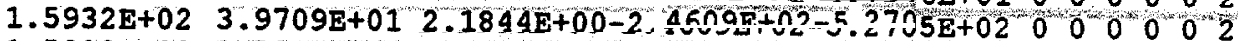
$\begin{array}{llllllll}1.5989 \mathrm{E}+02 & 3.5000 \mathrm{E}+01 & 7-5192 \mathrm{E}-01 & 2.4014 \mathrm{E}+00 & 5.9249 \mathrm{E}-010000 & 0 & 0 & 0\end{array}$ $1.6090 \mathrm{E}+023.5000 \mathrm{E}+0199,9260 \mathrm{E}-035.5700 \mathrm{E}+02-2.4520 \mathrm{E}+01000002$ $1.6093 \mathrm{E}+023.6434 \mathrm{E}+01,7.0994 \mathrm{E}+00-1.4140 \mathrm{E}+01-5.7185 \mathrm{E}-01 \quad 0 \quad 00001$ $1.6146 \mathrm{E}+02 \quad 3.5000 \mathrm{E}+01 \quad 3.7496 \mathrm{E}-02 \quad 1.5721 \mathrm{E}+02 \quad 5.3231 \mathrm{E}+0100000012$ $1.6225 \mathrm{E}+023.5000 \mathrm{E}+01 \quad 3.0652 \mathrm{E}-013.1744 \mathrm{E}+02$ 1.3954E+02 0000001 $1.6250 \mathrm{E}+02 \quad 3.5000 \mathrm{E}+019.8540 \mathrm{E}-039.2900 \mathrm{E}+00 \quad 1.3710 \mathrm{E}+01$ o $000 \mathrm{O}$

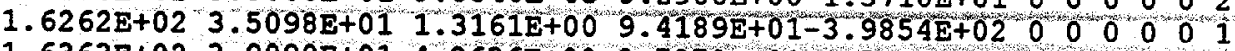
$1.6363 \mathrm{E}+023.9090 \mathrm{E}+01 \quad 4.2636 \mathrm{E}+00-2.7870 \mathrm{E}+01-1.5195 \mathrm{E}+02000002$ $1.6491 \mathrm{E}+02 \quad 3.5000 \mathrm{E}+01$ 1.5334E-01 3.2771E+02-8.3596E+010000 012 $1.6562 \mathrm{E}+024.2457 \mathrm{E}+01 \quad 3.0044 \mathrm{E}+00-4.2923 \mathrm{E}+01-4.0842 \mathrm{E}-0100002$

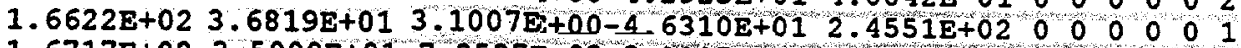
$\begin{array}{llllllllll}1.6717 \mathrm{E}+02 & 3.5000 \mathrm{E}+01 & 7.3537 \mathrm{E}-03 & 3.8097 \mathrm{E}+01 & 2.0987 \mathrm{E}+01 & 0 & 0 & 0 & 0 & 0\end{array}$ $1.6799 \mathrm{E}+02 \quad 3.8841 \mathrm{E}+01 \quad 3.8222 \mathrm{E}+00-7.3935 \mathrm{E}+00-3.2778 \mathrm{E}+02000002$

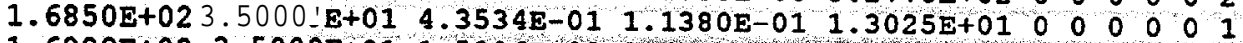
$1.6889 \mathrm{E}+02 \quad 3.5000 \mathrm{E}+01 \quad 1,5206 \mathrm{E}-02 \quad 2.6362 \mathrm{E}+01-1.3851 \mathrm{E}+00000002$ $\begin{array}{llllllll}1.6936 \mathrm{E}+02 & 3.5704 \mathrm{E}+01 & 2.5758 \mathrm{E}+00-8.3719 \mathrm{E}+01-1.3694 \mathrm{E}+02 & 0 & 0 & 0 & 0 & 1\end{array}$

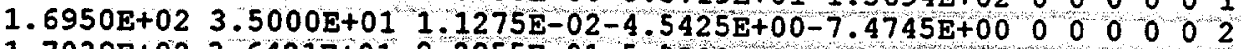
$1.7039 \mathrm{E}+023.6421 \mathrm{E}+01$ 9.2955E-01-5.5599E+01-3.5190E+01 0 o 000 o $1.7080 \mathrm{E}+023.5000 \mathrm{E}+017.5791 \mathrm{E}-02-3.5711 \mathrm{E}+01-4.0139 \mathrm{E}+01000002$ $\begin{array}{lllllllllllllllll}1.7119 \mathrm{E}+02 & 3.5000 \mathrm{E}+01 & 1.8664 \mathrm{E}-02 & 8.3357 \mathrm{E}+01 & 1.7592 \mathrm{E}+01 & 0 & 0 & 0 & 0 & 0 & 1 & 1\end{array}$ $1.7123 \mathrm{E}+023.5000 \mathrm{E}+01 \quad 1.8739 \mathrm{E}-01-2.6479 \mathrm{E}+02-2.2065 \mathrm{E}+02000002$ $1.7172 \mathrm{E}+02 \quad 3.4982 \mathrm{E}+01 \quad 1.2475 \mathrm{E}+00 \quad 9.7414 \mathrm{E}-01-2.5575 \mathrm{E}+010000001$ $\begin{array}{llllllllll}1.7217 \mathrm{E}+02 & 3.5000 \mathrm{E}+01 & 6.0271 \mathrm{E}-02 & 3.3308 \mathrm{E}+01-8.5378 \mathrm{E}+01 & 0 & 0 & 0 & 0 & 0 & 2\end{array}$ $1.7325 \mathrm{E}+023.5000 \mathrm{E}+01$ 1.8806E-01 1.9648E+02-1.5871E+02 000002 $1.7401 \mathrm{E}+024.1127 \mathrm{E}+011.8436 \mathrm{E}+00 \quad 1.6341 \mathrm{E}+02-1.5100 \mathrm{E}+01000002$

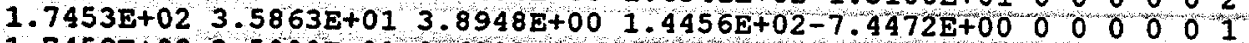
$1.7459 \mathrm{E}+023.5000 \mathrm{E}+019.6292 \mathrm{E}-022.2368 \mathrm{E}+02-1.3042 \mathrm{E}+020000002$ $1.7497 \mathrm{E}+02$ 3.6151E+01 $1.1140 \mathrm{E}+00-8.0623 \mathrm{E}+00-5.7686 \mathrm{E}+01000001$ $1.7523 \mathrm{E}+023.5000 \mathrm{E}+011.0790 \mathrm{E}-01-4.7840 \mathrm{E}+01-7.5667 \mathrm{E}+010000002$ $1.7571 \mathrm{E}+023.5000 \mathrm{E}+013.7137 \mathrm{E}-02-5.0264 \mathrm{E}+01-8.6669 \mathrm{E}+01000001$ 


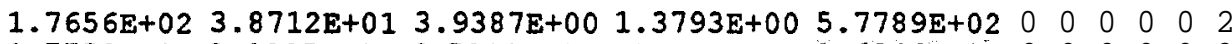

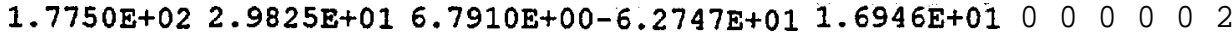
$\begin{array}{llllllllllll}1.7755 E+02 & 3.6991 E+01 & 3.3655 E+00 & 2.6279 E+01 & 6.3525 E+01 & 0 & 0 & 0 & 0 & 0 & 1\end{array}$

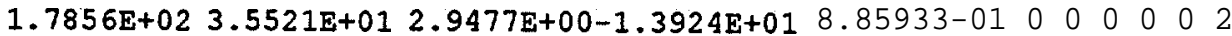
$1.7942 \mathrm{E}+023.1966 \mathrm{E}+01 \quad 1.7765 \mathrm{E}+00-7.7173 \mathrm{E}+00 \quad 7.97803-0300000000$

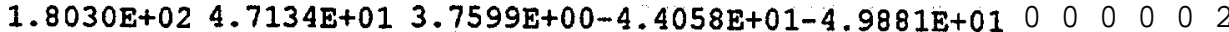
$1.8048 \mathrm{E}+02 \quad 3.5000 \mathrm{E}+01 \quad 3.0919 \mathrm{E}-02-4.5513 \mathrm{E}+012.3536 \mathrm{E}+02 \quad 0000001$

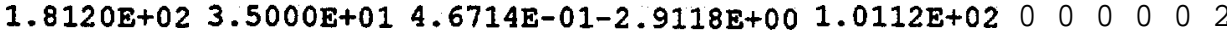

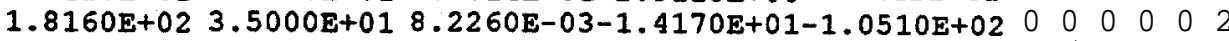

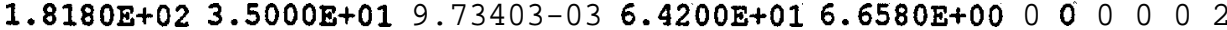

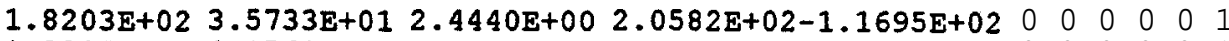

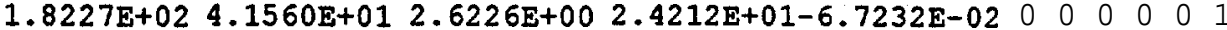
$\begin{array}{llllllllllll}1.8319 \mathrm{E}+02 & 3.5000 \mathrm{E}+01 & 5.53833-01 & 1.2160 \mathrm{E}+02 & 1.5677 \mathrm{E}+02 & 0 & 0 & 0 & 0 & 0 & 2\end{array}$ $\begin{array}{llllllllllll}1.8390 \mathrm{E}+02 & 3.5000 \mathrm{E}+01 & 9.72503-03 & 1.2150 \mathrm{E}+01 & 1.0450 \mathrm{E}+00 & 0 & 0 & 0 & 0 & 0 & 2\end{array}$

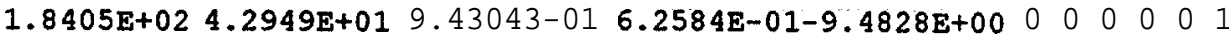
$1.8510 \mathrm{E}+02 \quad 3.5000 \mathrm{E}+01$ 5.2280E-04-1.7100E+01-2.5910E+01 00000002

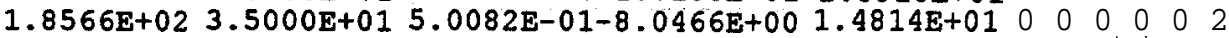
$1.8664 \mathrm{E}+023.2941 \mathrm{E}+019.5410 \mathrm{E}-01-4.2282 \mathrm{E}+02-5.1241 \mathrm{E}+010000000$ $1.8732 \mathrm{E}+023.5000 \mathrm{E}+01 \quad 5.02313-02 \quad 3.7404 \mathrm{E}+014.6581 \mathrm{E}+01 \quad 0^{\prime} \quad 0 \quad 0 \quad 0 \quad 0 \quad 1$ $\begin{array}{llllllllll}1.8770 \mathrm{E}+02 & 3.5000 \mathrm{E}+01 & 2.36253-01 & 6.8353 \mathrm{E}+01-3.5880 \mathrm{E}+02 & 0 & 0 & 0 & 0 & 0 & 1\end{array}$

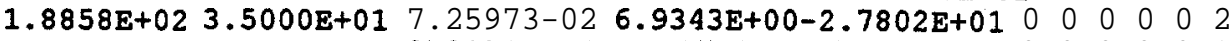
$\begin{array}{lllllllll}1.8891 \mathrm{E}+02 & 3.5000 \mathrm{E}+01 & 2.0134 \mathrm{E}-01-4.7899 \mathrm{E}+00-5.9981 \mathrm{E}+00 & 0 & 0 & 0 & 0 & 0 & 1\end{array}$

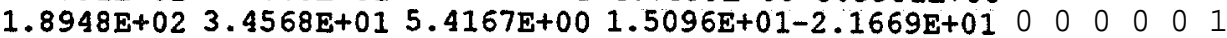

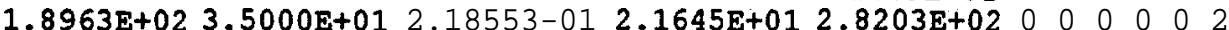

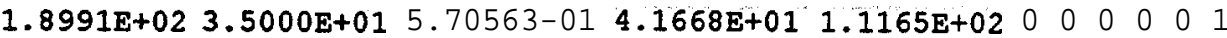

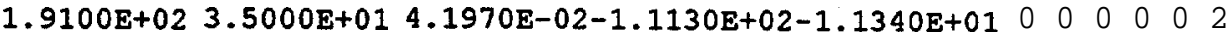
1.9140E+02 3.5000E+01 5.11903-02 2.4090E+01 7.5910E+01 00000002

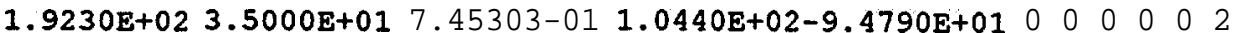
$1.9230 \mathrm{E}+02 \quad 3.5435 \mathrm{E}+018.7060 \mathrm{E}+00-1.7940 \mathrm{E}+014.7530 \mathrm{E}+010000001$ $\begin{array}{llllllllll}1.9300 \mathrm{E}+02 & 3.5000 \mathrm{E}+01 & 2.9730 \mathrm{E}-03-5.0210 \mathrm{E}+02 & 6.0180 \mathrm{E}+01 & 0 & 0 & 0 & 0 & 0 & 1\end{array}$

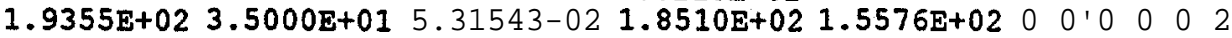

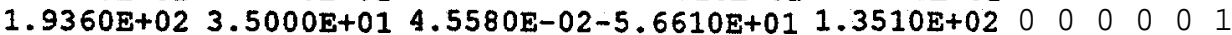

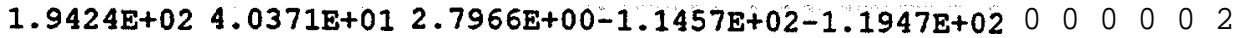

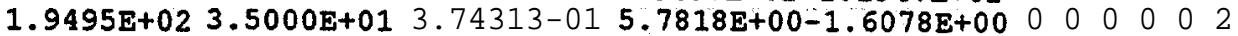
$\begin{array}{llllllllll}1.9577 \mathrm{E}+02 & 3.5000 \mathrm{E}+01 & 3.1592 \mathrm{E}-02-1.4752 \mathrm{E}+02 & 1.1671 \mathrm{E}+02 & 0 & 0 & 0 & 0 & 0 & 2\end{array}$

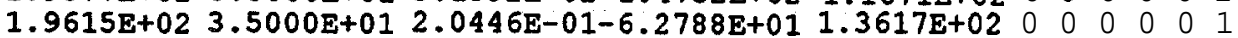

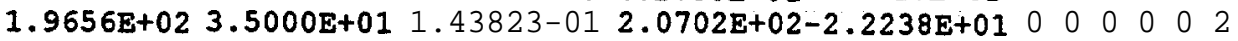

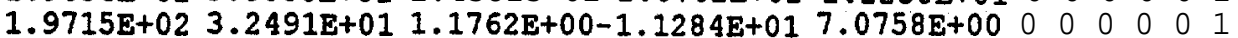

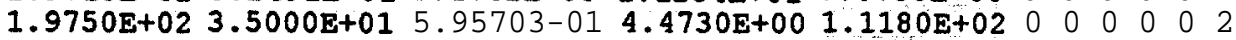

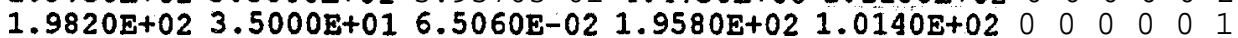
$1.9841 \mathrm{E}+023.3041 \mathrm{E}+01 \quad 1.7629 \mathrm{E}+00-1.8369 \mathrm{E}+02-1.3273 \mathrm{E}+02$ o $0 \mathrm{~b}-0 \mathrm{O} \quad 0 \quad 2$ $1.9882 \mathrm{E}+022.9762 \mathrm{E}+01 \quad 4.0365 \mathrm{E}+003.0996 \mathrm{E}+01-4.3349 \mathrm{E}+02 \quad 0000001$

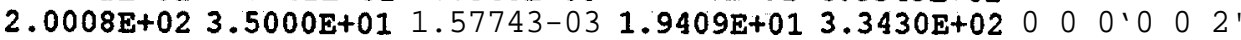

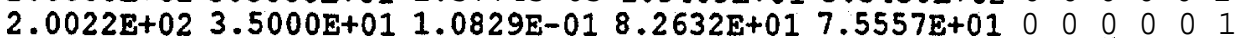

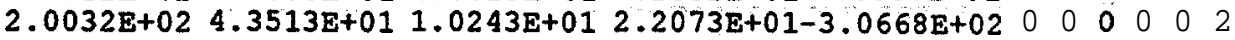

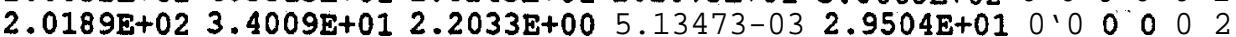

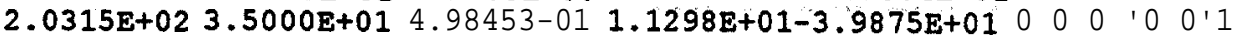
$2.0330 \mathrm{E}+023.5000 \mathrm{E}+01 \quad 5.6710 \mathrm{E}-03-6.7520 \mathrm{E}+001.2810 \mathrm{E}+0100001002$

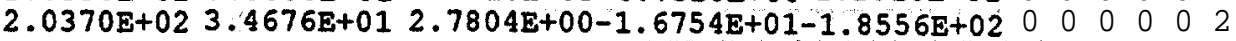

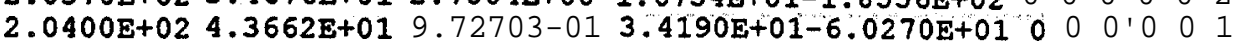

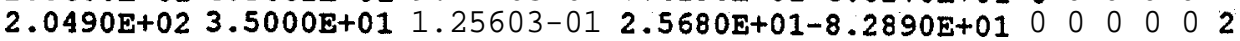

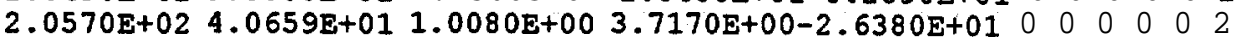
$\begin{array}{llllllllllllllll}2.0590 \mathrm{E}+02 & 3.5000 \mathrm{E}+01 & 1.8660 \mathrm{E}-01 & 9.2420 \mathrm{E}+01-8.7180 \mathrm{E}+01 & 0 & 0 & 0 & 0 & 0 & 1\end{array}$

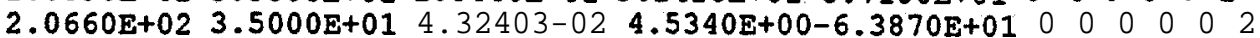
$2.0700 \mathrm{E}+02 \quad 3.5331 \mathrm{E}+01 \quad 4.5420 \mathrm{E}+00 \quad 6.2360 \mathrm{E}+00-1.5050 \mathrm{E}+02 \quad 0 \quad 000002$

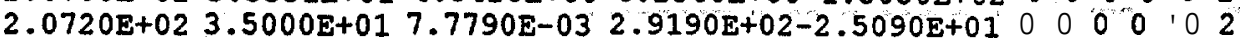

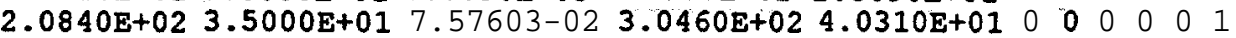

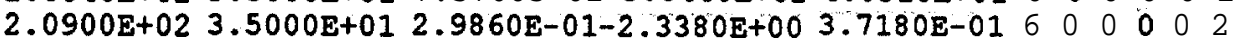

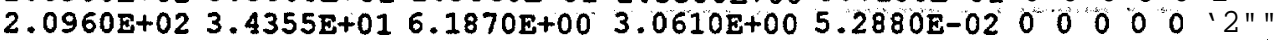
$2.1060 \mathrm{E}+02 \quad 4.2230 \mathrm{E}+01 \quad 1.7840 \mathrm{E}+00-9.9110 \mathrm{E}+01-6.5070 \mathrm{E}+01 \quad 0 \quad 0000001$ $2.1090 \mathrm{E}+02 \quad 3.5000 \mathrm{E}+01$ 1.0000E-02 2.0080E+00-4.9810E+00 00000002

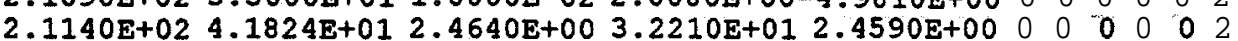
2.1200E+02 3.5000E+01 2.84203-01 $9.7940 \mathrm{E}+01-1.6500 \mathrm{E}+010000001$ $\begin{array}{lllllllllll}2.1220 \mathrm{E}+02 & 3.5000 \mathrm{E}+01 & 1.65703-01 & 2.6590 \mathrm{E}+01 & 1.9470 \mathrm{E}+01 & 0 & 0 & 0 & 0 & 0 & 1\end{array}$ 2.1270E+02 3.5000E+01 7.49103-01 $6.3910 \mathrm{E}+01-1.8910 \mathrm{E}+02 \quad 0 \quad 000002$ $2.1320 \mathrm{E}+023.5000 \mathrm{E}+019.08503-031.0650 \mathrm{E}+011.7000 \mathrm{E}+01000002$ 
2.1360E+02 3.4126E+01 6.6940E+00 $1.44503-01 \quad 1.4370 \mathrm{E}+02000000001$ $2.1380 \mathrm{E}+02 \quad 4.6241 \mathrm{E}+01 \quad 1.7890 \mathrm{E}+00-2.9390 \mathrm{E}+00-3.5740 \mathrm{E}+02 \quad 0000002$

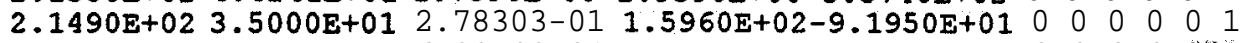

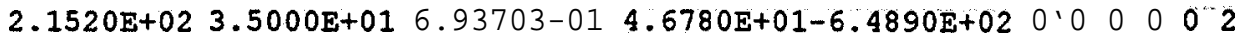

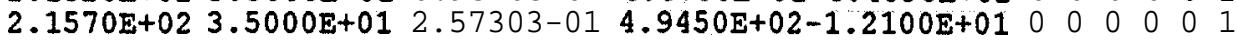
$2.1620 \mathrm{E}+02 \quad 3.5000 \mathrm{E}+01 \quad 1.0010 \mathrm{E}-02-1.5280 \mathrm{E}+00 \quad 4.2970 \mathrm{E}-02 \quad 0 \quad 00000 \mathrm{~b}-2$ 、 $2.1660 \mathrm{E}+02 \quad 3.5000 \mathrm{E}+013.63403-01 \quad 7.1790 \mathrm{E}+00-1.8900 \mathrm{E}+02 \quad 0000001$

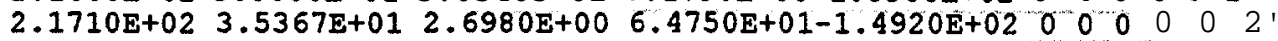
$2.1750 \mathrm{E}+02 \quad 3.5000 \mathrm{E}+01 \quad 1.0170 \mathrm{E}-02-3.9170 \mathrm{E}+01 \quad 1.1370 \mathrm{E}+02 \quad 00^{\circ} 00 \mathrm{~d}-2$ ' $2.1810 \mathrm{E}+02 \quad 3.5000 \mathrm{E}+01 \quad 6.15503-01 \quad 1.2230 \mathrm{E}+02-2.4960 \mathrm{E}+02 \quad 0 \quad 0 \quad 00001$ $2.1890 \mathrm{E}+02 \quad 3.5000 \mathrm{E}+01 \quad 4.1550 \mathrm{E}-01 \quad 1.7120 \mathrm{E}+02-3.0180 \mathrm{E}+02 \quad 0 \quad 000002$ $\begin{array}{llllllllllllll}2.1940 \mathrm{E}+02 & 3.5000 \mathrm{E}+01 & 1.3000 \mathrm{E}-02 & 9.4790 \mathrm{E}+01 & 8.2700 \mathrm{E}+01 & 0 & 0 & 0 & 0 & 0 & 2\end{array}$ $2.1967 \mathrm{E}+023.5000 \mathrm{E}+01 \quad 3.2614 \mathrm{E}-01 \quad 2.8743 \mathrm{E}+02-8.9746 \mathrm{E}+01$ o 01000001

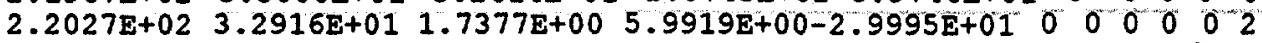
$2.2069 \mathrm{E}+02 \quad 3.9437 \mathrm{E}+01 \quad 1.0411 \mathrm{E}+01 \quad 3.4453 \mathrm{E}+02 \quad 1.0121 \mathrm{E}+02 \quad 0 \quad 0-O \quad 0 \quad 01$

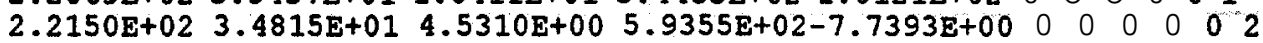

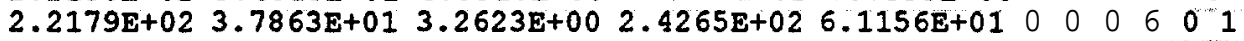

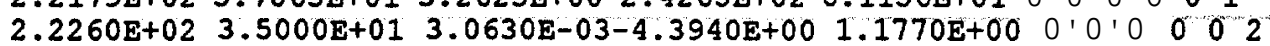

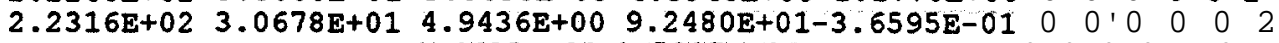

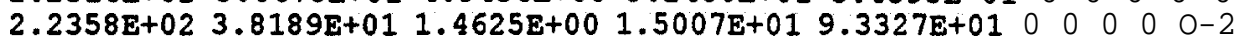

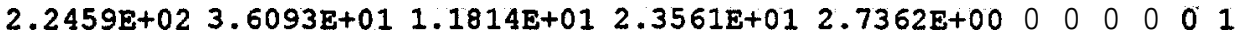

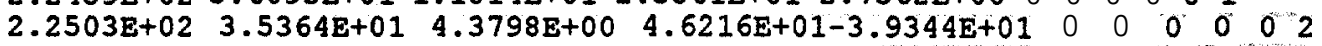
$\begin{array}{llllllllllllllllllllllll}2.2525 \mathrm{E}+02 & 3.8145 \mathrm{E}+01 & 8.31603-01 & 7.8592 \mathrm{E}+00 & 6.4542 \mathrm{E}+0 & 0 & 0 & 0 & 0 & 0 & 1\end{array}$ $2.2546 \mathrm{E}+02 \quad 3.5000 \mathrm{E}+01 \quad 2.6564 \mathrm{E}-01-3.1231 \mathrm{E}+02 \quad 4.7264 \mathrm{E}+02 \quad 0 \quad 000002$

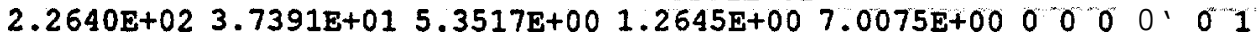

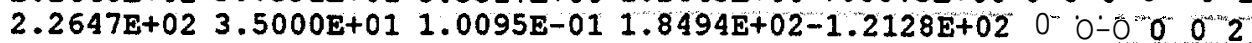
$\begin{array}{llllllllllllll}2.2695 \mathrm{E}+02 & 3.4105 \mathrm{E}+01 & 3.9950 \mathrm{E}+00 & 2.5577 \mathrm{E}+02-5.5994 \mathrm{E}+02 & 0 & 0 & 0 & 0 & 0 & 2\end{array}$ $2.2877 \mathrm{E}+023.7175 \mathrm{E}+01 \quad 1.5796 \mathrm{E}+00 \quad 9.6333 \mathrm{E}+01 \quad 4.3854 \mathrm{E}+01 \quad 0 \quad 0 \quad 6 \quad 6 \quad 0=1$ $2.2917 \mathrm{E}+02 \quad 3.2995 \mathrm{E}+01 \quad 1.1464 \mathrm{E}+00 \quad 1.7778 \mathrm{E}+01-8.2097 \mathrm{E}+000001002$

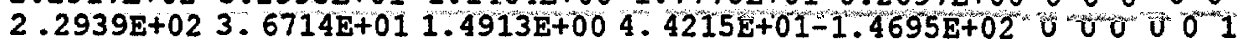
$2.2960 \mathrm{E}+02 \quad 3.5000 \mathrm{E}+01 \quad 6.3790 \mathrm{E}-04 \quad 4.3830 \mathrm{E}+01-9.6970 \mathrm{E}+01$ o 0 o 002 $2.3057 \mathrm{E}+023.5000 \mathrm{E}+018.7173 \mathrm{E}-03 \quad 6.2906 \mathrm{E}+00-1.9062 \mathrm{E}+01$ o 0 o 0.02 $2.3092 \mathrm{E}+023.8137 \mathrm{E}+01$ 1.1317 $\mathrm{E}+011.0956 \mathrm{E}+00-1.9313 \mathrm{E}+01000001$ $2.3150 \mathrm{E}+023.7130 \mathrm{E}+018.0666 \mathrm{E}+003.1087 \mathrm{E}+02 \quad 1.5645 \mathrm{E}+00$ o 000002 $2.3292 \mathrm{E}+02 \quad 3.1996 \mathrm{E}+01 \quad 4.4259 \mathrm{E}+00 \quad 5.7520 \mathrm{E}-01 \quad 1.1001 \mathrm{E}+02 \quad 0 \quad 0 \quad 0 \quad 002$ $2.3306 \mathrm{E}+02 \quad 4.0020 \mathrm{E}+01 \quad 1.0473 \mathrm{E}+00 \quad 4.7593 \mathrm{E}+02-1.7164 \mathrm{E}+02$ o 00 o 01 $2.3362 \mathrm{E}+023.5000 \mathrm{E}+012.1289 \mathrm{E}-023.0645 \mathrm{E}+01-5.6083 \mathrm{E}+01$ o o 0 o 02

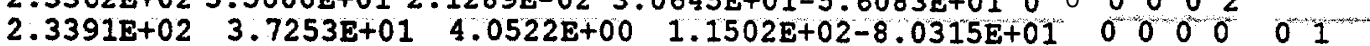

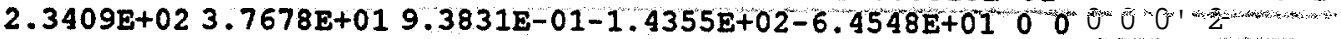

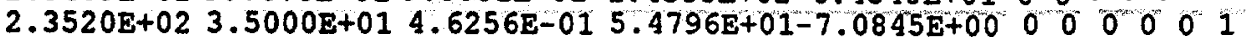
$2.3614 \mathrm{E}+023.5000 \mathrm{E}+012.7965 \mathrm{E}-03$ 9.2043E+01-7.4626E+01 000002

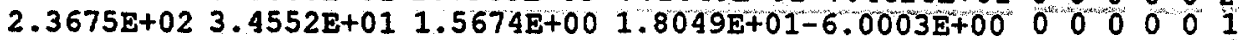
$\begin{array}{llllllllllllllllllll}2.3719 E+02 & 3.5000 \mathrm{E}+01 & 9.34213-03 & 2.1860 \mathrm{E}+02-1.6826 \mathrm{E}+02 & 0 & 0 & 0 & 0 & 0\end{array}$ $2.3785 \mathrm{E}+023.5000 \mathrm{E}+012.7353 \mathrm{E}-028.3806 \mathrm{E}+012.0187 \mathrm{E}+02000002$ $2.3813 \mathrm{E}+02 \quad 3.0077 \mathrm{E}+01 \quad 1.0936 \mathrm{E}+00 \quad 1.5195 \mathrm{E}+02-2.1572 \mathrm{E}+02 \quad 0 \quad 000001$ $2.3869 \mathrm{E}+02 \quad 3.4045 \mathrm{E}+01 \quad 1.1289 \mathrm{E}+00 \quad 2.7601 \mathrm{E}+02-2.5563 \mathrm{E}+02 \quad 0 \quad 0 \quad 0 \quad 0 \quad 02$ $2.3913 \mathrm{E}+023.3866 \mathrm{E}+01 \quad 1.6952 \mathrm{E}+002.3177 \mathrm{E}+02-1.9898 \mathrm{E}+00000 \mathrm{O}$ $2.3938 \mathrm{E}+023.2161 \mathrm{E}+013.9612 \mathrm{E}+003.9311 \mathrm{E}+02 \quad 4.1018 \mathrm{E}+02000001$ $2.3971 \mathrm{E}+023.5000 \mathrm{E}+018.7905 \mathrm{E}-033.3608 \mathrm{E}+015.5137 \mathrm{E}+02$ 1 300002 $2.4079 \mathrm{E}+02 \quad 3.5000 \mathrm{E}+01 \quad 4.3396 \mathrm{E}-02 \quad 6.0247 \mathrm{E}+00-2.1357 \mathrm{E}+02 \quad 0$ a 0002 $2.4118 \mathrm{E}+023.4497 \mathrm{E}+018.2361 \mathrm{E}+001.0716 \mathrm{E}+02-3.2698 \mathrm{E}+0200002$ $2.4201 \mathrm{E}+023.5000 \mathrm{E}+016.2334 \mathrm{E}-02-6.0366 \mathrm{E}+003.568 \mathrm{E}+010000 \mathrm{0}$ $2.4280 \mathrm{E}+023.2918 \mathrm{E}+013.3262 \mathrm{E}+00-3.4173 \mathrm{E}+02-4.2002 \mathrm{E}+020000001$ $2.4319 \mathrm{E}+023.5000 \mathrm{E}+01 \quad 4.1730 \mathrm{E}-01-1.9802 \mathrm{E}+02 \quad 7.1254 \mathrm{E}+00000002$ $2.4361 \mathrm{E}+023.5000 \mathrm{E}+01 \quad 4.0957 \mathrm{E}-012.7301 \mathrm{E}+01-1.0693 \mathrm{E}+02 \quad 0 \quad 0 \quad 00001$ $2.4439 \mathrm{E}+02 \quad 3.5000 \mathrm{E}+01 \quad 6.3496 \mathrm{E}-014.0278 \mathrm{E}+02 \quad 2.2858 \mathrm{E}+01$ o 010002 $2.4545 \mathrm{E}+023.2782 \mathrm{E}+012.7901 \mathrm{E}+003.3584 \mathrm{E}+01-1.7354 \mathrm{E}+02000002$ $2.4631 \mathrm{E}+02 \quad 3.8859 \mathrm{E}+01 \quad 3.7827 \mathrm{E}+00-6.3028 \mathrm{E}+01-3.578 \mathrm{E}+02 \mathrm{O} 0 \mathrm{O} 0 \mathrm{O}$ $2.4653 \mathrm{E}+023.5000 \mathrm{E}+012.14783-02 \quad 2.5371 \mathrm{E}+02 \quad 2.6133 \mathrm{E}-010000000$

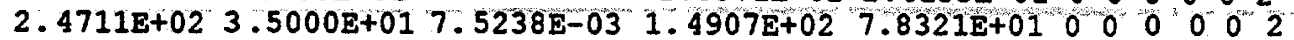
$\begin{array}{llllllllllllllllllll}2.4781 \mathrm{E}+02 & 3.8325 \mathrm{E}+01 & 5.3750 \mathrm{E}+00 & 2.3370 \mathrm{E}+00-2.9055 \mathrm{E}+01 & 0 & 0 & 0 & 0 & 0 & 2\end{array}$ $2.4796 \mathrm{E}+023.4772 \mathrm{E}+011.5816 \mathrm{E}+00-3.0020 \mathrm{E}+02-2.7022 \mathrm{E}+02$ o 00001 $\begin{array}{lllllllllllll}2.4870 \mathrm{E}+02 & 3.5000 \mathrm{E}+01 & 2.42203-02 & 3.2915 \mathrm{E}+02-4.6286 \mathrm{E}+02 & 0 & 0 & 0 & 0 & 0 & 2\end{array}$ $2.4876 \mathrm{E}+02 \quad 3.5000 \mathrm{E}+01 \quad 5.3843 \mathrm{E}-01 \quad 2.2828 \mathrm{E}+02 \quad 3.6481 \mathrm{E}+01 \quad 0 \quad 0 \quad 0.001$

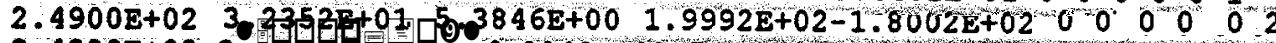
$2.4980 \mathrm{E}+023$ $6.0040 \mathrm{E}-027.6360 \mathrm{E}+01-7.8890 \mathrm{E}+0100001$ 


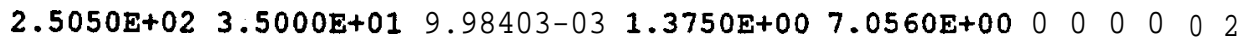

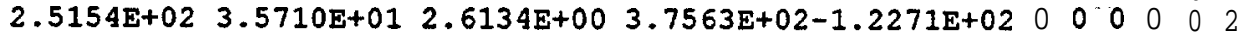
$2.5258 \mathrm{E}+02 \quad 3.5000 \mathrm{E}+01 \quad 1.0416 \mathrm{E}-02-1.9353 \mathrm{E}+01-7.0271 \mathrm{E}+0202000001$

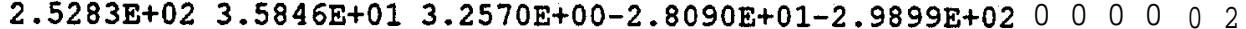

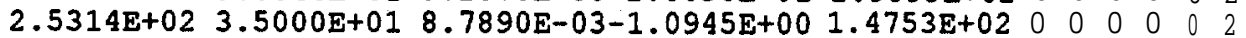
$2.5366 \mathrm{E}+02 \quad 3.6322 \mathrm{E}+01 \quad 9.5701 \mathrm{E}+00 \quad 4.0419 \mathrm{E}+02-2.4330 \mathrm{E}+020000001$

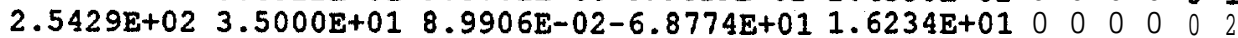

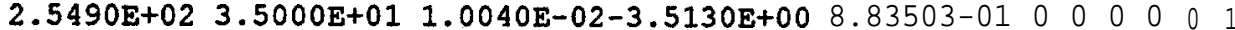

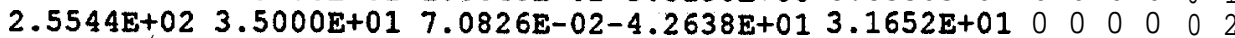

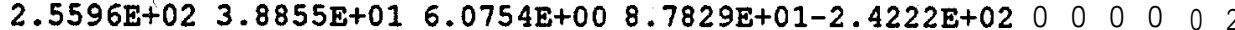

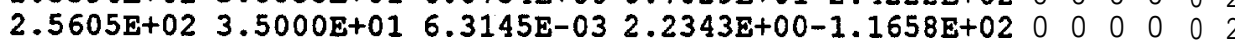

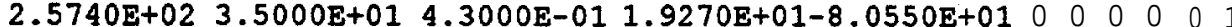
$2.5780 \mathrm{E}+02 \quad 3.5000 \mathrm{E}+01$ 9.9610E-03-7.3570E-01-2.9490E-01 00000002

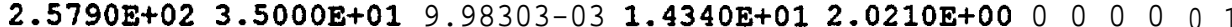

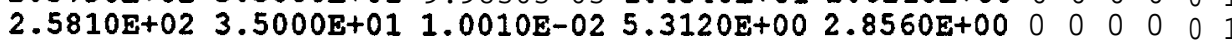

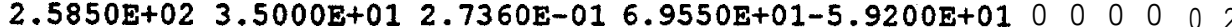

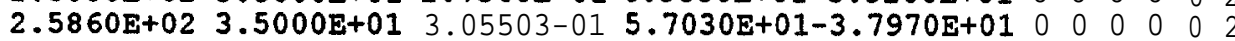

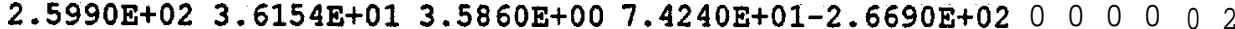
$2.6009 \mathrm{E}+02 \quad 3.5000 \mathrm{E}+01 \quad 4.1443 \mathrm{E}-04 \quad 4.8173 \mathrm{E}+02-4.1803 \mathrm{E}+02 \quad 000000001$

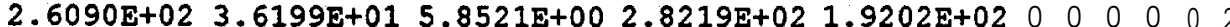

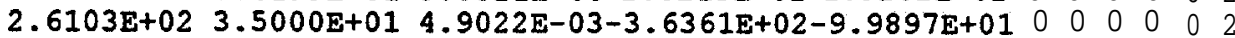

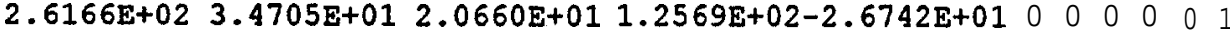

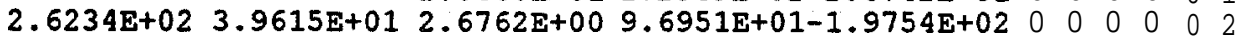

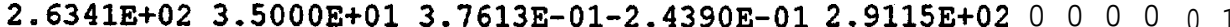

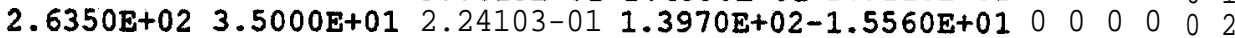

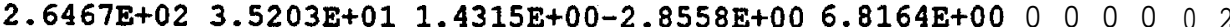
$2.6521 \mathrm{E}+02 \quad 3.5000 \mathrm{E}+01 \quad 1.1626 \mathrm{E}-02-1.5882 \mathrm{E}+02 \quad 4.89 .05 \mathrm{E}+020000001$

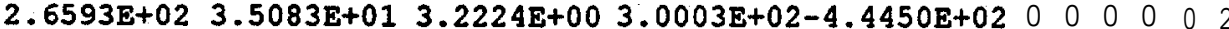

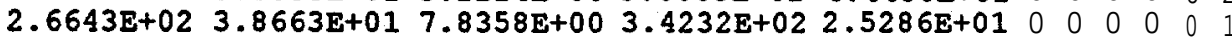

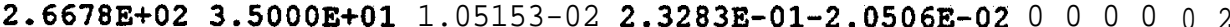

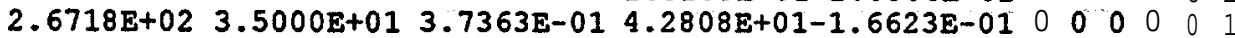

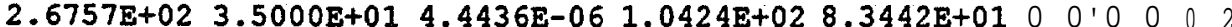

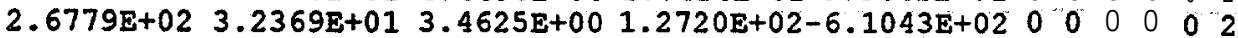
$2.6780 \mathrm{E}+023.5000 \mathrm{E}+01$ 9.9870E-03-5.5370E+00 2.5710E+00 0100001

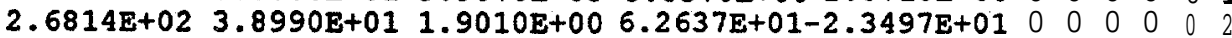

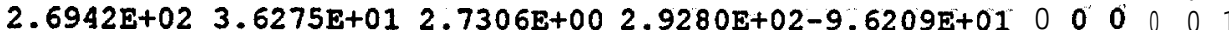

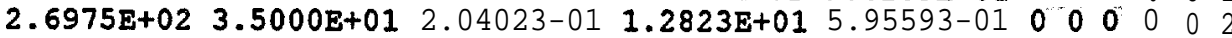

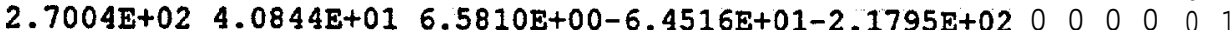

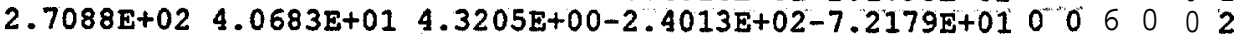
$\begin{array}{llllllllllll}2.7182 \mathrm{E}+02 & 3.5000 \mathrm{E}+01 & 3.1641 \mathrm{E}-03 & 3.3960 \mathrm{E}+02 & 1.1185 \mathrm{E}+02 & 0 & 0 & 0 & 0 & 0 & 2\end{array}$ $\begin{array}{llllllllllllll}2.7258 \mathrm{E}+02 & 3.5000 \mathrm{E}+01 & 1.25303-03 & 2.9743 \mathrm{E}+00 & 2.2469 \mathrm{E}+02 & 0 & 0 & 0 & 0 & 0 & 2\end{array}$

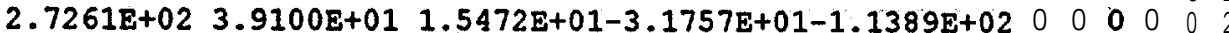

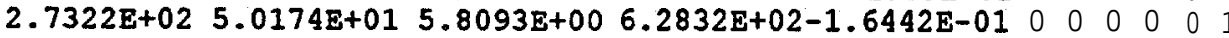

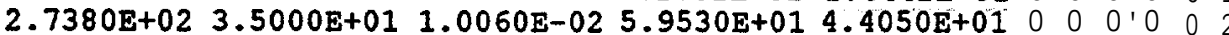

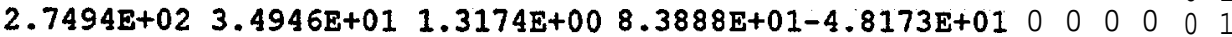

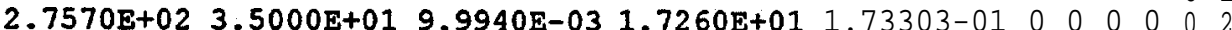
$2.7630 \mathrm{E}+02 \quad 3.5000 \mathrm{E}+01$ 9.9900E-03-8.8320E+00 1.5230E+01 00000003

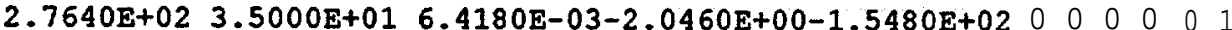

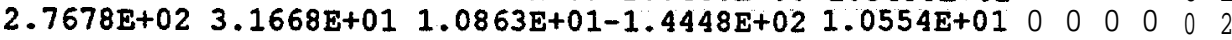

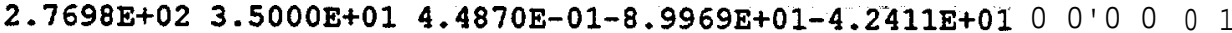
$2.7787 \mathrm{E}+023.5000 \mathrm{E}+017.8603 \mathrm{E}-01-2.2386 \mathrm{E}+015.6743 \mathrm{E}+00^{\circ} 0^{-1} 0{ }^{\prime} \mathrm{a}^{\prime} \quad 0 \quad 02$

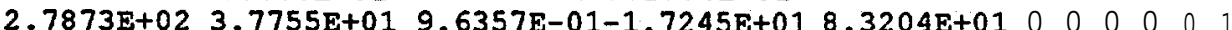

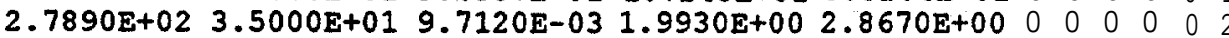

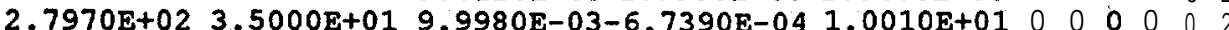
$2.7983 \mathrm{E}+02 \quad 3.1910 \mathrm{E}+01 \quad 1.2415 \mathrm{E}+01 \quad 5.3489 \mathrm{E}+02-2.2222 \mathrm{E}+02 \quad 01000001$

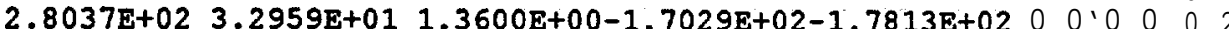
$2.8063 \mathrm{E}+02 \quad 3.9142 \mathrm{E}+01 \quad 1.0469 \mathrm{E}+001.8268 \mathrm{E}+02-1.5694 \mathrm{E}+0200000001$

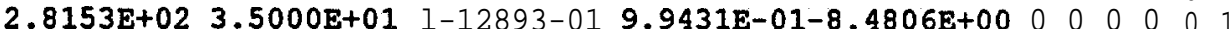

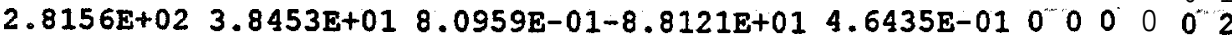

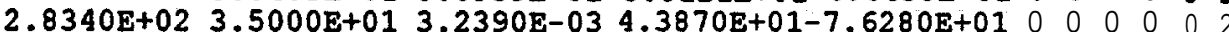

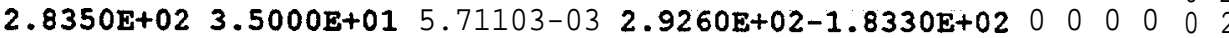

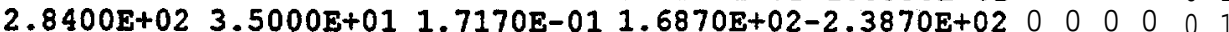

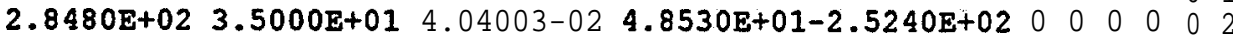

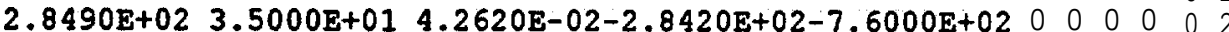

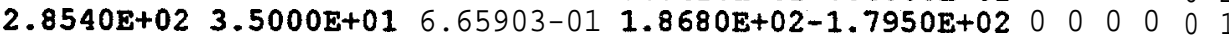

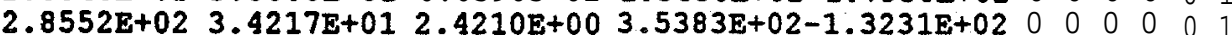


$\begin{array}{llllllllllll}2.8580 \mathrm{E}+02 & 3.5000 \mathrm{E}+01 & 2.9280 \mathrm{E}-03 & 4.6100 \mathrm{E}+02 & 8.1990 \mathrm{E}+01 & 0 & 0 & 0 & 0 & 0 & 2\end{array}$ $2.8603 \mathrm{E}+02 \quad 3.5000 \mathrm{E}+01 \quad 3.4025 \mathrm{E}-01-3.0204 \mathrm{E}+01-1.2179 \mathrm{E}+020000001$ $2.8650 \mathrm{E}+02 \quad 3.5000 \mathrm{E}+012.1400 \mathrm{E}-02-1.4000 \mathrm{E}+02-4.0410 \mathrm{E}+020000010$ $2.8747 \mathrm{E}+02 \quad 3.2943 \mathrm{E}+01 \quad 3.5931 \mathrm{E}+00 \quad 1.0311 \mathrm{E}+02-2.1700 \mathrm{E}+02 \quad 0 \quad 0 \quad 0 \quad 0 \quad 02$ $2.8850 \mathrm{E}+023.5000 \mathrm{E}+019.9880 \mathrm{E}-03 \quad 1.7070 \mathrm{E}+00-9.7690 \mathrm{E}-01$ o 000002

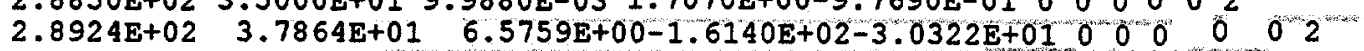
$2.8972 \mathrm{E}+023.6185 \mathrm{E}+014.3589 \mathrm{E}+00-1.2704 \mathrm{E}+02-1.7157 \mathrm{E}+020^{2} 0_{0} 0^{\circ} 011$

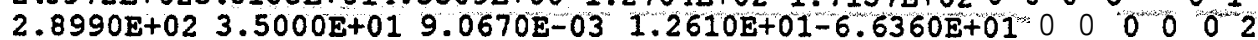
$\begin{array}{lllllllllllll}2.9029 \mathrm{E}+02 & 3.5540 \mathrm{E}+01 & 3.5670 \mathrm{E}+00 & 1.2127 \mathrm{E}+02-1.2280 \mathrm{E}+0 \mathrm{~W} & 0 & 0 & 0 & 0 & 1\end{array}$ $2.9117 \mathrm{E}+02 \quad 3.5000 \mathrm{E}+012.63513-01 \quad 1.3280 \mathrm{E}+02-3.8704 \mathrm{E}+00$ o 000 o 011 $2.9221 \mathrm{E}+02 \quad 3.5822 \mathrm{E}+01 \quad 2.1970 \mathrm{E}+00 \quad 1.6197 \mathrm{E}+02 \quad 3.1610 \mathrm{E}+02 \quad 0 \quad 000001$

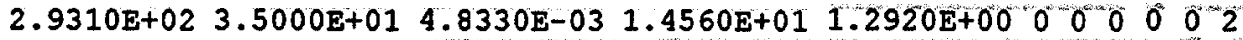
$2.9352 \mathrm{E}+02 \quad 4.3587 \mathrm{E}+01 \quad 8.7010 \mathrm{E}-01 \quad 2.8428 \mathrm{E}-01-8.4401 \mathrm{E}+01$ o 0 o 0 o $2.9410 \mathrm{E}+023.5000 \mathrm{E}+012.6606 \mathrm{E}-02-3.0413 \mathrm{E}+01-3.9640 \mathrm{E}+02000002$

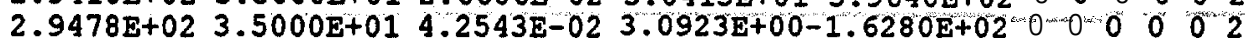
$\begin{array}{lllllllllllllllllll}2.9532 \mathrm{E}+02 & 3.6735 \mathrm{E}+01 & 2.5645 \mathrm{E}+00 & 2.7166 \mathrm{E}+02 & 3.0094 \mathrm{E}+02 & 0 & 0 & 0 & 0 & 0 & 1\end{array}$ $2.9549 \mathrm{E}+023.5000 \mathrm{E}+011.3746 \mathrm{E}-02-5.3710 \mathrm{E}+01-3.0075 \mathrm{E}+02=0.0 .002$ $2.9604 \mathrm{E}+023.4494 \mathrm{E}+01$ 1.8042E+00-7.1013E+01-4.6221E+01 0 o 0 o 02 $2.9619 \mathrm{E}+023.5000 \mathrm{E}+011.50213-029.1793 \mathrm{E}+00-6.6394 \mathrm{E}-016^{\prime} 6^{\prime} 0001$ $2.9731 \mathrm{E}+02 \quad 4.5536 \mathrm{E}+01 \quad 8.2574 \mathrm{E}-01 \quad 1.1771 \mathrm{E}+01 \quad 7.1860 \mathrm{E}+01 \quad 0 \quad 000102$ $2.9775 \mathrm{E}+02 \quad 3.5000 \mathrm{E}+01 \quad 1.3033 \mathrm{E}-02-4.7085 \mathrm{E}+02-5.3307 \mathrm{E}+01$ o 00 o 00 o 1 $\begin{array}{llllllllllllll}2.9820 \mathrm{E}+02 & 3.5000 \mathrm{E}+01 & 7.0764 \mathrm{E}-03-4.0935 \mathrm{E}+01-2.2025 \mathrm{E}+02 & 0 & 0 & 0 & 0 & 0 & 2\end{array}$

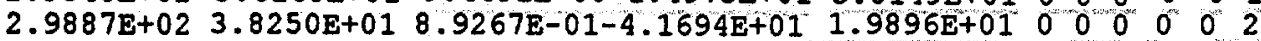
$2.9990 \mathrm{E}+023.5000 \mathrm{E}+018.1338 \mathrm{E}-02-6.3074 \mathrm{E}+003.8398 \mathrm{E}+01$ o 0 o 0 o 0 o 2 $3.0043 \mathrm{E}+023.5000 \mathrm{E}+014.1643 \mathrm{E}-01-1.0702 \mathrm{E}+02-5.9232 \mathrm{E}+01000001$ 3.0053E+02 3.5000E+01 2.10873-01' 5.9712E+01 7.5934E+01 o ow 00 o 2 $3.0107 \mathrm{E}+023.5000 \mathrm{E}+011.4097 \mathrm{E}-01 \quad 1.2860 \mathrm{E}+02 \quad 5.8701 \mathrm{E}+010000001$ $3.0173 \mathrm{E}+02 \quad 3.5000 \mathrm{E}+016.1205 \mathrm{E}-01 \quad 5.5025 \mathrm{E}+01 \quad 6.0741 \mathrm{E}+01$ o 0 o 002 $3.0251 \mathrm{E}+023.5000 \mathrm{E}+012.0716 \mathrm{E}-01 \quad 1.1592 \mathrm{E}+022.1482 \mathrm{E}+02 \quad 00000$ $3.0274 \mathrm{E}+02 \quad 3.6606 \mathrm{E}+01 \quad 1.7505 \mathrm{E}+003.1257 \mathrm{E}+002.4072 \mathrm{E}+02000001$

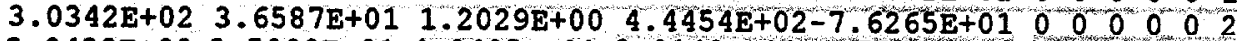
$3.0438 \mathrm{E}+023.5000 \mathrm{E}+014.0405 \mathrm{E}-012.0140 \mathrm{E}+02-1.5560 \mathrm{E}+02$ o 00001 $3.0474 \mathrm{E}+02 \quad 3.6202 \mathrm{E}+01 \quad 9.2463 \mathrm{E}-01 \quad 1.0640 \mathrm{E}+022.1558 \mathrm{E}+01$ o 000002 $3.0506 \mathrm{E}+023.1737 \mathrm{E}+012.4266 \mathrm{E}+001.0383 \mathrm{E}+02-2.6075 \mathrm{E}+02 \cdots 00002$ $\begin{array}{llllllllllllllllllll}3.0559 \mathrm{E}+02 & 3.9044 \mathrm{E}+01 & 1.7194 \mathrm{E}+00 & 1.6417 \mathrm{E}+02 & 1.8040 \mathrm{E}+01 & 0 & 0 & 0 & 0 & 0 & 1\end{array}$ $3.0576 \mathrm{E}+023.5000 \mathrm{E}+012^{\prime} 2381 \mathrm{E}-012.3523 \mathrm{E}+023.7598 \mathrm{E}+0200000 \mathrm{0}$

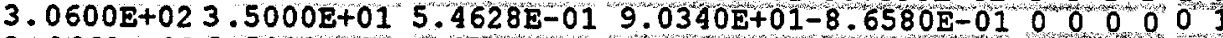
3.0660E+02 3.5000E+013.6560E-01 1.2030E+02-1.2070E+02 0 000 o 02 $3.0710 \mathrm{E}+02 \quad 3.5000 \mathrm{E}+01 \quad 3.7910 \mathrm{E}-01 \quad 5.8540 \mathrm{E}+01-2.8170 \mathrm{E}+01000001$, $3.0750 \mathrm{E}+023.5000 \mathrm{E}+014.5036 \mathrm{E}-01 \quad 6.3780 \mathrm{E}+01 \quad 1.9860 \mathrm{E}+00$ o 000002

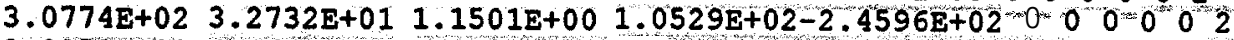
$3.0858 \mathrm{E}+02 \quad 4.1644 \mathrm{E}+01 \quad 8.6440 \mathrm{E}-01 \quad 2.2262 \mathrm{E}+02-1.2149 \mathrm{E}+02000001$ $3.0894 \mathrm{E}+02 \quad 3.3358 \mathrm{E}+01 \quad 3.6079 \mathrm{E}+00 \quad 8.2603 \mathrm{E}+00 \quad 2.6397 \mathrm{E}+000000002$ $3.0979 \mathrm{E}+02 \quad 3.5000 \mathrm{E}+01 \quad 1.7984 \mathrm{E}-01 \quad 4.1472 \mathrm{E}+01-9.5402 \mathrm{E}+01$ o 000 o 01 $\begin{array}{llllllllllllllll}3.1034 \mathrm{E}+02 & 3.5000 \mathrm{E}+01 & 2.3406 \mathrm{E}-01 & 3.6392 \mathrm{E}+01 & 1.6340 \mathrm{E}+01 & 0 & 0 & 0 & 0 & 0 & 2\end{array}$ $3.1129 \mathrm{E}+02 \quad 3.2922 \mathrm{E}+01 \quad 1.2410 \mathrm{E}+00 \quad 6.0713 \mathrm{E}+01-7.0367 \mathrm{E}+01000000$ $3.1151 \mathrm{E}+02 \quad 3.5000 \mathrm{E}+01 \quad 8.4691 \mathrm{E}-03-2.6328 \mathrm{E}+01-5.7294 \mathrm{E}+01 \quad 0 \quad 000002$ $3.1232 \mathrm{E}+02 \quad 3.8766 \mathrm{E}+01 \quad 1.2522 \mathrm{E}+00 \quad 1.5414 \mathrm{E}+02-1.1241 \mathrm{E}+02 \quad 0 \quad 0 \quad 0 \quad 0 \quad 0 \quad 2$ $3.1245 \mathrm{E}+02 \quad 3.8496 \mathrm{E}+01 \quad 1.3727 \mathrm{E}+00 \quad 9.1914 \mathrm{E}+01-6.5192 \mathrm{E}+01$ o 0 o 0 o 02 $3.1318 \mathrm{E}+02 \quad 3.8675 \mathrm{E}+01 \quad 5.0124 \mathrm{E}+00 \quad 1.3744 \mathrm{E}+012.2415 \mathrm{E}+00000001$ $3.1354 \mathrm{E}+02 \quad 2.8504 \mathrm{E}+01 \quad 1.6085 \mathrm{E}+00-3.5035 \mathrm{E}+01 \quad 1.2328 \mathrm{E}+02$ a 00002 $3.1498 \mathrm{E}+02 \quad 3.5422 \mathrm{E}+01$ 1.2526E+00 $7.0705 \mathrm{E}+01-1.0457 \mathrm{E}+02$ o 0 o 0 o 1 $3.1504 \mathrm{E}+023.1636 \mathrm{E}+01 \quad 3.7909 \mathrm{E}+00-1.8585 \mathrm{E}-01 \quad 6.9830 \mathrm{E}+01$ o 000002 $3.1567 \mathrm{E}+02 \quad 3.6355 \mathrm{E}+013.3559 \mathrm{E}+00 \quad 1.0210 \mathrm{E}+02-2.7347 \mathrm{E}+01 \quad 0$ o 0000 $3.1601 \mathrm{E}+02 \quad 3.4835 \mathrm{E}+01 \quad 2.0040 \mathrm{E}+00 \quad 8.0234 \mathrm{E}+01 \quad 7.1140 \mathrm{E}+01 \quad 0 \quad 0 \quad 0001$ $3.1622 \mathrm{E}+02.3 .6607 \mathrm{E}+011.8776 \mathrm{E}+001.6599 \mathrm{E}+02-2.3132 \mathrm{E}+02$ o 0 o 0002 $3.1717 \mathrm{E}+02 \quad 3.0897 \mathrm{E}+01 \quad 1.4641 \mathrm{E}+002.7150 \mathrm{E}+01 \quad 1.0219 \mathrm{E}+02000001$ $3.1760 \mathrm{E}+02 \quad 3.2041 \mathrm{E}+019.0520 \mathrm{E}-01 \quad 2.2530 \mathrm{E}+01-2.1260 \mathrm{E}+0100002$ $3.1868 \mathrm{E}+02 \quad 3.5000 \mathrm{E}+012.9458 \mathrm{E}-018.0783 \mathrm{E}+01-1.0708 \mathrm{E}+02 \quad 000002$ $3.1892 \mathrm{E}+02 \quad 3.9907 \mathrm{E}+01$ 8.0109E-01 $1.0658 \mathrm{E}+02-1.3101 \mathrm{E}+02$ o 000001 $\begin{array}{llllllllllllllllll}3.1939 \mathrm{E}+02 & 3.6627 \mathrm{E}+01 & 8.6224 \mathrm{E}-01 & 1.7132 \mathrm{E}+02-2.4822 \mathrm{E}+02 & 0 & 0 & 0 & 0 & 0 & 2\end{array}$ $3.1964 \mathrm{E}+02 \quad 4.6762 \mathrm{E}+01 \quad 3.3707 \mathrm{E}+00 \quad 9.8802 \mathrm{E}+01-6.1846 \mathrm{E}+01$ o 000001 $3.2114 \mathrm{E}+02 \quad 3.5000 \mathrm{E}+01 \quad 4.3115 \mathrm{E}-01 \quad 3.1634 \mathrm{E}+02-1.0725 \mathrm{E}+01$ o 0 o 00002 $3.2161 \mathrm{E}+023.5000 \mathrm{E}+012.4970 \mathrm{E}-011.7682 \mathrm{E}+01-1.6902 \mathrm{E}+02000001$ 
$3.2204 \mathrm{E}+028.7753 \mathrm{E}+017.0138 \mathrm{E}-01-9.4342 \mathrm{E}+01-6.4940 \mathrm{E}+0000000002$ $\begin{array}{lllllllllllllll}3.2257 \mathrm{E}+02 & 2.8019 \mathrm{E}+01 & 2.9984 \mathrm{E}+00-1.4940 \mathrm{E}+00-2.0000 \mathrm{E}-01 & 0 & 0 & 0 & 0 & 0 & 2 & \\ 3.2269 \mathrm{E}+02 & 2.1467 \mathrm{E}+01 & 7.7823 \mathrm{E}+00-3.8124 \mathrm{E}+02 & 3.0827 \mathrm{E}+02 & 0 & 0 & 0 & 0 & 0 & 1\end{array}$ $\begin{array}{lllllllllll}3.2316 \mathrm{E}+02 & 3.5000 \mathrm{E}+01 & 6.2970 \mathrm{E}-01-2.2195 \mathrm{E}+01 & 2.4378 \mathrm{E}+01 & 0 & 0 & 0 & 0 & 0 & 2\end{array}$

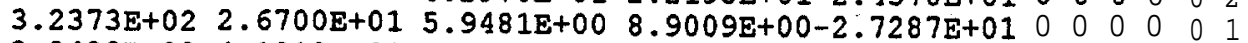

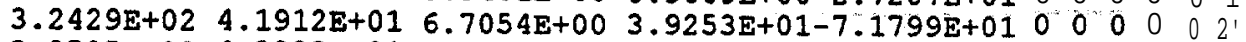

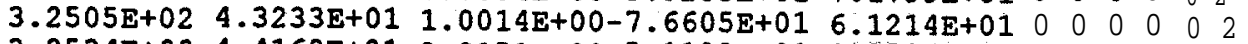
$\begin{array}{lllllllllll}3.2534 \mathrm{E}+02 & 4.4162 \mathrm{E}+01 & 2.9151 \mathrm{E}+00 & 5.1182 \mathrm{E}+01-1.3506 \mathrm{E}+01 & 0 & 0 & 0 & 0 & 0 & 1\end{array}$ $\begin{array}{lllllllllll}3.2596 \mathrm{E}+02 & 3.6556 \mathrm{E}+01 & 4.8440 \mathrm{E}+00-9.0259 \mathrm{E}+01 & 2.5713 \mathrm{E}+00 & 0 & 0 & 0 & 0 & 0 & 2\end{array}$

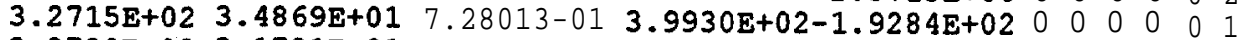

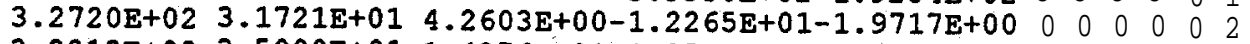

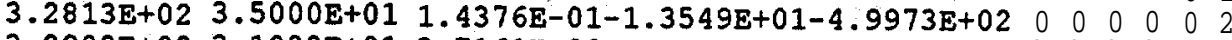
$\begin{array}{lllllllllll}3.2908 \mathrm{E}+02 & 3.1028 \mathrm{E}+01 & 3.7161 \mathrm{E}+00 & 7.3099 \mathrm{E}+01-1.5945 \mathrm{E}+02 & 0 & 0 & 0 & 0 & 0 & 1 & \\ 3.2913 \mathrm{E}+02 & 3.5000 \mathrm{E}+01 & 1.7504 \mathrm{E}-01-1.2240 \mathrm{E}+02 & 3.9430 \mathrm{E}+01 & 0 & 0 & 0 & 0 & 0 & 12\end{array}$

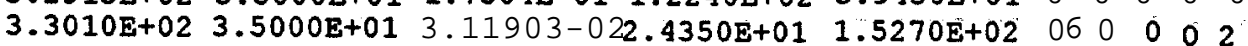
$3.3064 \mathrm{E}+02 \quad 3.8230 \mathrm{E}+012.6501 \mathrm{E}+00 \quad 1.2925 \mathrm{E}+02-4.7067 \mathrm{E}+02 \quad 0 \quad 010002$

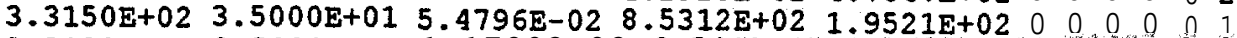

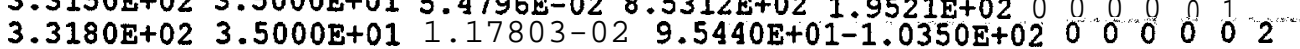
$\begin{array}{lllllllllll}3.3250 \mathrm{E}+02 & 3.7281 \mathrm{E}+01 & 4.5324 \mathrm{E}+00 & 5.2447 \mathrm{E}+01-9.9208 \mathrm{E}+01 & 0 & 0 & 0 & 0 & 0 & 1\end{array}$

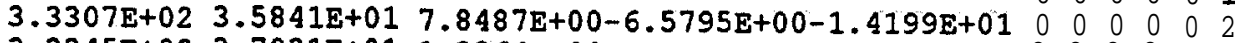

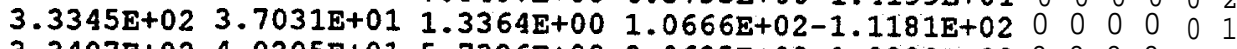

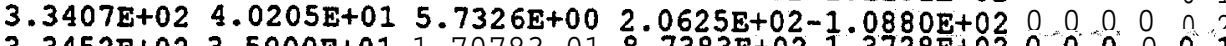

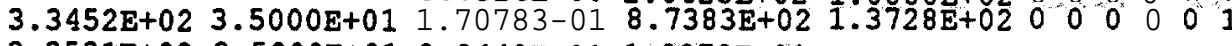

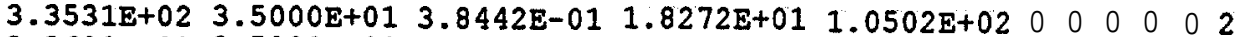

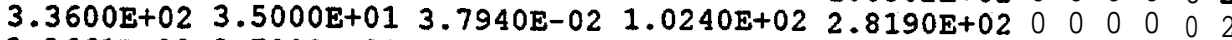

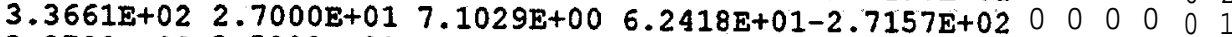

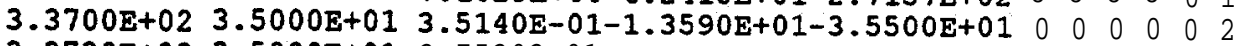
3.3730E+02 3.5000E+01 3.75303-01 6.5060E+00-5.5280E-01 000100001

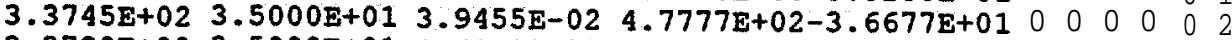
$\begin{array}{llllllllllll}3.3790 \mathrm{E}+02 & 3.5000 \mathrm{E}+01 & 2.62503-01 & \mathbf{8} .2190 \mathrm{E}+01-9.0380 \mathrm{E}+01 & 0 & 0 & 0 & 0 & 0 & 1\end{array}$

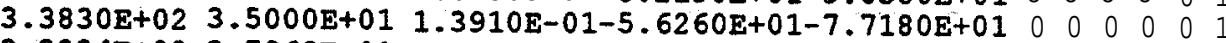
$3.3834 \mathrm{E}+02 \quad 3.7969 \mathrm{E}+01 \quad 1.2583 \mathrm{E}+00 \quad 1.1775 \mathrm{E}+02-1.0074 \mathrm{E}+0200001002$

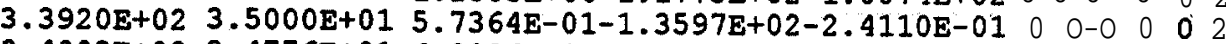

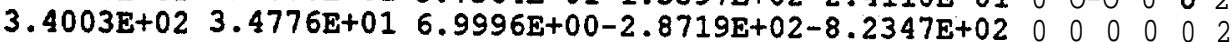

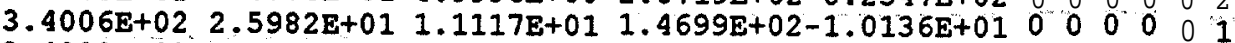
$\begin{array}{lllllllllllll}3.4090 \mathrm{E}+02 & 3.5000 \mathrm{E}+01 & 3.25003-01 & 2.2530 \mathrm{E}+02-2.7220 \mathrm{E}+00 & 0 & 0 & 0 & 0 & 0 & 2\end{array}$

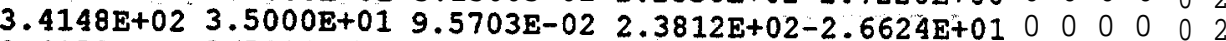
$\begin{array}{llllllllllll}3.4150 \mathrm{E}+02 & 3.5000 \mathrm{E}+01 & 2.50903-01 & 2.4200 \mathrm{E}+01-8.7770 \mathrm{E}+01 & 0 & 0 & 0 & 0 & 0 & 1\end{array}$ $\begin{array}{lllllllllll}3.4223 \mathrm{E}+02 & 4.1848 \mathrm{E}+01 & 2.5658 \mathrm{E}+00 & 2.5275 \mathrm{E}+01-1.9679 \mathrm{E}+01 & 0 & 0 & 0 & 0 & 0 & 2 \\ 3.4315 \mathrm{E}+02 & 3.7934 \mathrm{E}+01 & 3.4825 \mathrm{E}+00 & 8.5143 \mathrm{E}+00 & 1.6000 \mathrm{E}-01 & 0 & 0 & 0 & 0 & 0 & 2\end{array}$

$\begin{array}{llllllllllllllllll}3.4360 \mathrm{E}+02 & 3.7905 \mathrm{E}+01 & 8.0439 \mathrm{E}+00 & 9.8657 \mathrm{E}+02 & 1.7348 \mathrm{E}+02 & 0 & 0 & 0 & 0\end{array}$

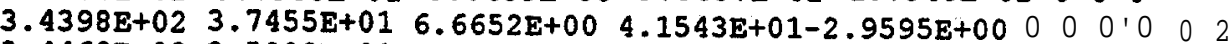

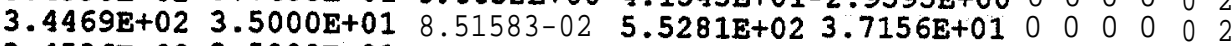
$\begin{array}{lllllllllll}3.4526 \mathrm{E}+02 & 3.5000 \mathrm{E}+01 & 1.5119 \mathrm{E}-01 & 2.3469 \mathrm{E}+02-7.2275 \mathrm{E}+00 & 0 & 0 & 0 & 0 & 0 & 1\end{array}$

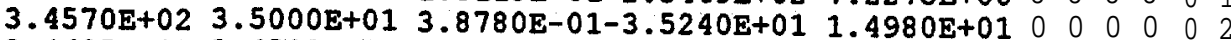

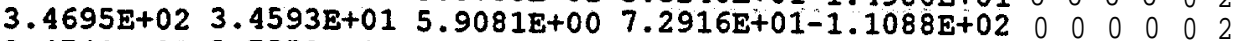

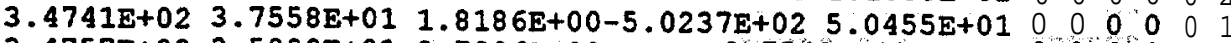

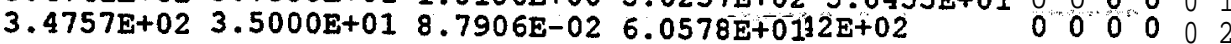
$\begin{array}{llllllllllll}3.4818 \mathrm{E}+02 & 3.6133 \mathrm{E}+01 & 2.1881 \mathrm{E}+00 & 3.8589 \mathrm{E}+01-4.4437 \mathrm{E}+00 & 0 & 0 & 0 & 0 & 0 & 2 \\ 3.4936 \mathrm{E}+02 & 3.4411 \mathrm{E}+01 & 1.3881 \mathrm{E}+00 & 2.7058 \mathrm{E}+01 & 3.3632 \mathrm{E}+02 & 0 & 0 & 0 & 0 & 0 & 2\end{array}$ $\begin{array}{ccccccccccc}3.4936 \mathrm{E}+02 & 3.4411 \mathrm{E}+01 & 1.3881 \mathrm{E}+00 & 2.7058 \mathrm{E}+01 & 3.3632 \mathrm{E}+02 & 0 & 0 & 0 & 0 & 0 & 2 \\ 3.4942 \mathrm{E}+02 & 3.5000 \mathrm{E}+01 & 1.49403-01 & 4.1910 \mathrm{E}+01-5.5459 \mathrm{E}+01 & 0 & 0 & 0 & 0 & 0 & 1\end{array}$

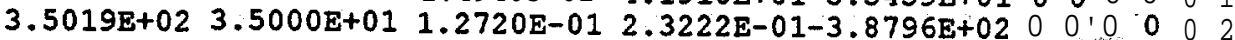

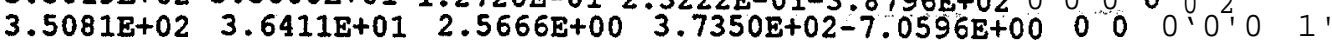

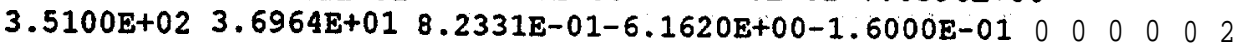

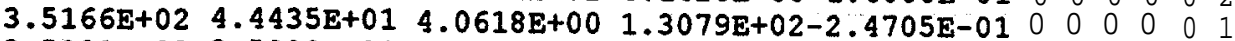

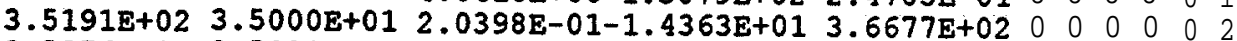

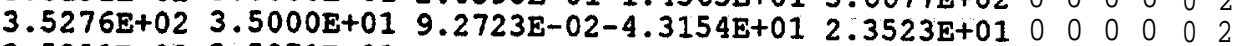

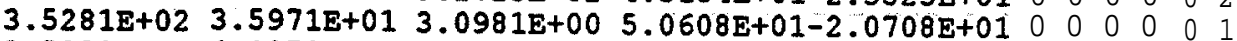

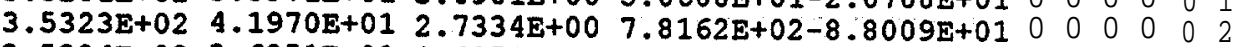

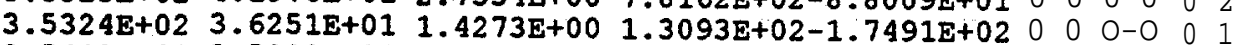

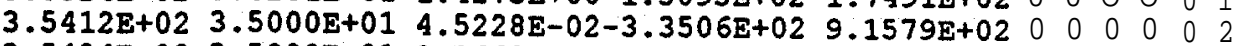

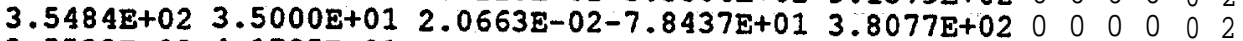
$\begin{array}{llllllllllll}3.5533 \mathrm{E}+02 & 4.1795 \mathrm{E}+01 & 6.5826 \mathrm{E}+00 & 3.7288 \mathrm{E}+02-2.7411 \mathrm{E}+00 & 0 & 0 & 0 & 0 & 0 & 1 & \\ 3.5605 \mathrm{E}+02 & 3.4554 \mathrm{E}+01 & 3.6292 \mathrm{E}+00 & 6.8875 \mathrm{E}+00-2.3999 \mathrm{E}+00 & 0 & 0 & 0 & 0 & 0 & 2\end{array}$ $\begin{array}{cccccccccc}3.5605 \mathrm{E}+02 & 3.4554 \mathrm{E}+01 & 3.6292 \mathrm{E}+00 & 6.8875 \mathrm{E}+00-2.3999 \mathrm{E}+00 & 0 & 0 & 0 & 0 & 0 & 2 \\ 3.5641 \mathrm{E}+02 & 3.5000 \mathrm{E}+01 & 1.3461 \mathrm{E}-01-5.8917 \mathrm{E}+02-2.2142 \mathrm{E}+02 & 0 & 0 & 0 & 0 & 0 & 2\end{array}$

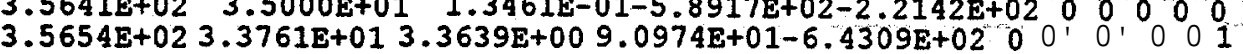


3.5747E+02 3.3356E+01 $8.27203-01 \quad 1.0668 \mathrm{E}+02-2.6235 \mathrm{E}+02 \quad 0 \quad 000002$

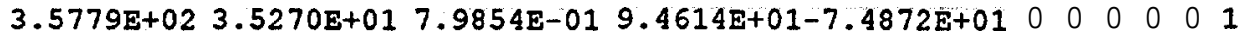

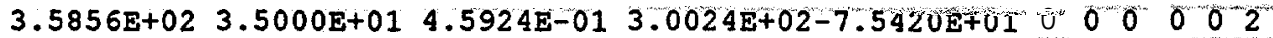
$3.5874 \mathrm{E}+02 \quad 3.5000 \mathrm{E}+01 \quad 2.3654 \mathrm{E}-01 \quad 1.7521 \mathrm{E}+02-9.3745 \mathrm{E}+01 \quad 0 \quad 0 \quad 0 \quad 6 \quad 0 \quad 1$ $3.5960 \mathrm{E}+02 \quad 4.3979 \mathrm{E}+01 \quad 1.8702 \mathrm{E}+00 \quad 1.6337 \mathrm{E}+02-1.5516 \mathrm{E}+01 \quad 0 \quad 000002$ $\begin{array}{lllllllllllll}3.5980 \mathrm{E}+02 & 3.8980 \mathrm{E}+01 & 7.80903-01 & 5.2660 \mathrm{E}+00 & 1.3480 \mathrm{E}+02 & 0 & 0 & 0 & 0 & 0 & 1\end{array}$

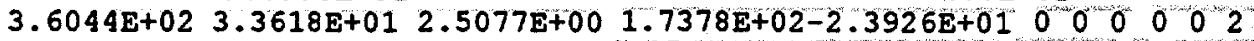

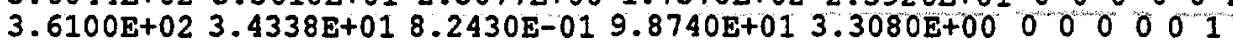

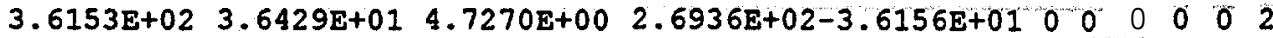
$3.6249 \mathrm{E}+02 \quad 4.9283 \mathrm{E}+01 \quad 1.5547 \mathrm{E}+001.6732 \mathrm{E}+02-2.4979 \mathrm{E}+01 \quad 0 \quad 000601$, $3.6269 \mathrm{E}+02 \quad 3.5000 \mathrm{E}+012.1796 \mathrm{E}-01 \quad 8.0365 \mathrm{E}+01 \quad 7.0138 \mathrm{E}+010000000$

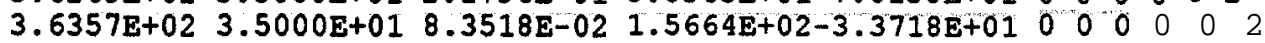
$3.6414 \mathrm{E}+02 \quad 3.6274 \mathrm{E}+01 \quad 2.2632 \mathrm{E}+00 \quad 1.3435 \mathrm{E}+02-4.8789 \mathrm{E}+02 \quad 0 \quad 0^{\prime} 00^{\prime} 00^{\prime} 1$ $3.6448 \mathrm{E}+02 \quad 3.6794 \mathrm{E}+012.0114 \mathrm{E}+00 \quad 2.6332 \mathrm{E}+00-3.4381 \mathrm{E}+01 \quad 00001002$ $3.6515 \mathrm{E}+023.7067 \mathrm{E}+014.7992 \mathrm{E}+009.2751 \mathrm{E}+01-2.3310 \mathrm{E}+026^{\prime} 0$ o $60^{2} 1$

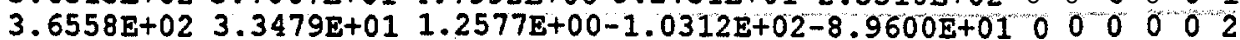
$3.6593 \mathrm{E}+023.6715 \mathrm{E}+01 \quad 1.6245 \mathrm{E}+00-6.7594 \mathrm{E}+01-1.4493 \mathrm{E}+02 \quad 0 \quad 06061$ $3.6690 \mathrm{E}+023.5000 \mathrm{~B}+016.4907 \mathrm{E}-015.8450 \mathrm{E}+00-1.8500 \mathrm{E}+01 \mathrm{O}+\mathrm{s}$ $3.6794 \mathrm{E}+02 \quad 3.5000 \mathrm{E}+016.9282 \mathrm{E}-01-3.0131 \mathrm{E}+02-3.1031 \mathrm{E}+02 \quad 0100002$ $3.6863 \mathrm{E}+02 \quad 4.1517 \mathrm{E}+01$ 1.6364E+00-1.8363E+02-2.8772E+02 00 10 00102

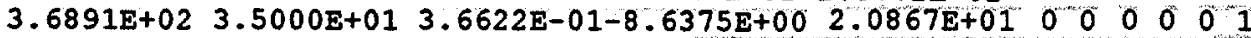

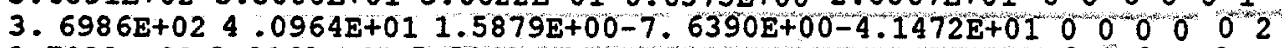
$3.7032 \mathrm{E}+023.2163 \mathrm{E}+015.5063 \mathrm{E}+00-1.4103 \mathrm{E}+01-1.5544 \mathrm{E}+0100000-2^{\prime}$ $3.7137 \mathrm{E}+02 \quad 4.1286 \mathrm{E}+01 \quad 3.1760 \mathrm{E}+00 \quad 8.5034 \mathrm{E}+01-1.8806 \mathrm{E}+02$ o 00 o 001 $3.7143 \mathrm{E}+02 \quad 3.5000 \mathrm{E}+01 \quad 1.4666 \mathrm{E}-02-4.1397 \mathrm{E}+01 \quad 8.7136 \mathrm{E}+01$ o 0100102 $3.7260 \mathrm{E}+02 \quad 3.1591 \mathrm{E}+013.2298 \mathrm{E}+00 \quad 2.6095 \mathrm{E}+00 \quad 2.0427 \mathrm{E}+000000002$ $3.7265 \mathrm{E}+023.5000 \mathrm{E}+017.0485 \mathrm{E}-03-1.6749 \mathrm{E}+015.3396 \mathrm{E}+01$ o 000.01

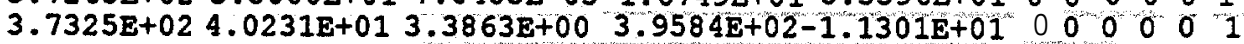

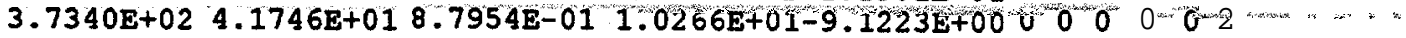
$3.7484 \mathrm{E}+02 \quad 3.5000 \mathrm{E}+013.1479 \mathrm{E}-01 \quad 1.0343 \mathrm{E}+02-1.2149 \mathrm{E}+02000002$ $3.7546 \mathrm{E}+023.5000 \mathrm{E}+012.6401 \mathrm{E}-012.8699 \mathrm{E}+02 \quad 7.9794 \mathrm{E}+01 \quad 0$ o 00 o 02 $3.7586 \mathrm{E}+02 \quad 3.5000 \mathrm{E}+013.0535 \mathrm{E}-02 \quad 5.4478 \mathrm{E}+01-5.5883 \mathrm{E}+01 \quad 0 \quad 0 \quad 0001$ $\begin{array}{lllllllllllllll}3.7647 \mathrm{E}+02 & 3.5000 \mathrm{E}+01 & 3.8942 \mathrm{E}-01-2.2676 \mathrm{E}+02-1.5552 \mathrm{E}+02 & 0 & 0 & 0 & 0 & 0 & 2\end{array}$ $3.7731 \mathrm{E}+023.5000 \mathrm{E}+013.7076 \mathrm{E}-021.5085 \mathrm{E}+01-2.1579 \mathrm{E}+010000001$ $3.7755 \mathrm{E}+023.4343 \mathrm{E}+01 \quad 1.1560 \mathrm{E}+001.0454 \mathrm{E}+02-1.2888 \mathrm{E}+02000002$ $3.7783 \mathrm{E}+023.9811 \mathrm{E}+013.4496 \mathrm{E}+001.3896 \mathrm{E}+02-1.4228 \mathrm{E}+0200001$ $3.7850 \mathrm{E}+023.5000 \mathrm{E}+013.1805 \mathrm{E}-01-2.2799 \mathrm{E}+021.8938 \mathrm{E}+01$ o 00 o 0 o 2

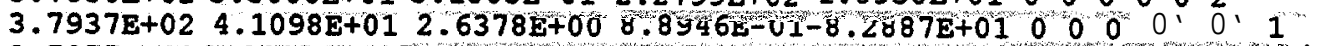
$3.7977 \mathrm{E}+022.9907 \mathrm{E}+017.8311 \mathrm{E}+00-1.6000 \mathrm{E}-01-3.8906 \mathrm{E}+02000 \mathrm{O} 0 \mathrm{O}$ $\begin{array}{llllllllllllll}3.8023 \mathrm{E}+02 & 4.2329 \mathrm{E}+01 & 2.9306 \mathrm{E}+00 & 2.9463 \mathrm{E}+00 & 2.0179 \mathrm{E}+01 & 0 & 0 & 0 & 0 & 0 & 2\end{array}$ $3.8132 \mathrm{E}+023.5000 \mathrm{E}+015.6868 \mathrm{E}-02.7 .7991 \mathrm{E}+002.8997 \mathrm{E}+01 \quad 0 \quad 00002$

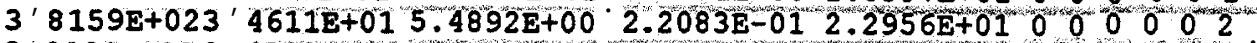
$3.8191 \mathrm{E}+023.4338 \mathrm{E}+011.1356 \mathrm{E}+001.0224 \mathrm{E}+01-1.6043 \mathrm{E}+020000001$

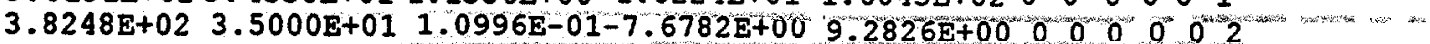
$3.8320 \mathrm{E}+023.5000 \mathrm{E}+01$ 1.5706E-01-4.8997E+00-2.2479E+00 o 0 o 0 o 011 $3.8332 \mathrm{E}+023.8138 \mathrm{E}+015.4555 \mathrm{E}+002.5620 \mathrm{E}+02-4.2052 \mathrm{E}+01$ o 00002 $3.8431 \mathrm{E}+02 \quad 3.5000 \mathrm{E}+019.6658 \mathrm{E}-02-5.4737 \mathrm{E}+01-1.0861 \mathrm{E}+02$ o 00002 $3^{\prime} 8442 \mathrm{E}+024^{\prime} 1668 \mathrm{E}+01$ 1.5549E+00-7.9752E+00 1.6630 $3.8492 \mathrm{E}+023.5000 \mathrm{E}+01$ 1.0084E-01 $4.8482 \mathrm{E}+01-8.5385 \mathrm{E}+01$ o 00002 $3.8516 \mathrm{E}+023.5000 \mathrm{E}+015.2303 \mathrm{E}-014.3391 \mathrm{E}+022.1492 \mathrm{E}+02$ o 0000 o $3^{\prime} 8517 \mathrm{E}+023^{\prime} 6301 \mathrm{E}+018^{\prime} 5441 \mathrm{E}-017.4321 \mathrm{E}+0 \mathrm{~T}-1,3249 \mathrm{E}+02^{2} 0^{2} 00000$ $3.8697 \mathrm{E}+024.3825 \mathrm{E}+01 \quad 1.4471 \mathrm{E}+00-3.8271 \mathrm{E}+004.0004 \mathrm{E}+0000000$

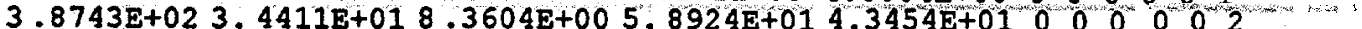
$\begin{array}{lllllllllllllllll}3.8829 \mathrm{E}+02 & 3.5008 \mathrm{E}+01 & 3.8495 \mathrm{E}+00-7.8752 \mathrm{E}+01 & 1.7465 \mathrm{E}+01 & 0 & 0 & 0 & 0 & 1\end{array}$ $3.8838 \mathrm{E}+023.5000 \mathrm{E}+01 \quad 8.3924 \mathrm{E}-05-8.4853 \mathrm{E}+01-3.5375 \mathrm{E}+01$ o 00 o 0 o 2

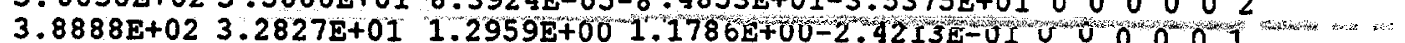
$3.8949 \mathrm{E}+023.5000 \mathrm{E}+014.2316 \mathrm{E}-029.1199 \mathrm{E}+01-4.2074 \mathrm{E}+0 \mathrm{~T}$ o 0 o 02 $3.9070 \mathrm{E}+023.5000 \mathrm{E}+011.9022 \mathrm{E}-019.3847 \mathrm{E}-011.7849 \mathrm{E}+000$ o o 0102 $3.9115 \mathrm{E}+023.5000 \mathrm{E}+017.0079 \mathrm{E}-02-5.1385 \mathrm{E}+01-6.1021 \mathrm{E}+0100001$ $3.9174 \mathrm{E}+023.5000 \mathrm{E}+013.8421 \mathrm{E}-03-1.0533 \mathrm{E}+01 \mathrm{~T}^{2} .5835 \mathrm{E}+02$ o 0 on on 3. $9220 \mathrm{E}+023.4224 \mathrm{E}+017.6129 \mathrm{E}+00 \mathrm{5} .3369 \mathrm{E}+01-1.1140 \mathrm{E}+02$ o 0 o 0.02 $3.9281 \mathrm{E}+023.5000 \mathrm{E}+014.7772 \mathrm{E}-012.0988 \mathrm{E}+025.0948 \mathrm{E}+01$ o 00002 $\begin{array}{lllllllllllllllll}3.9340 \mathrm{E}+02 & 3.1776 \mathrm{E}+01 & 2.0038 \mathrm{E}+00 & 8.9275 \mathrm{E}+01 & 3.8776 \mathrm{E}+02 & 0 & 0 & 0 & 0 & 0 & 1\end{array}$

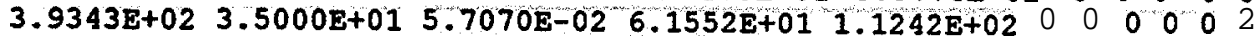
$3.9404 \mathrm{E}+02$ 3.5000E+01 1.3811E-01 1.7689E+02 2.4970E+02 Q ' 000002 $3.9430 \mathrm{E}+023^{-1} 5000 \mathrm{E}+015.3309 \mathrm{E}-011.8280 \mathrm{E}+02-1.3470 \mathrm{E}+01$ o 00001 
$3.9487 \mathrm{E}+02 \quad 3.5000 \mathrm{E}+01 \quad 4.8528 \mathrm{E}-012.7006 \mathrm{E}+02-2.1203 \mathrm{E}+02 \quad 0 \quad 000002$

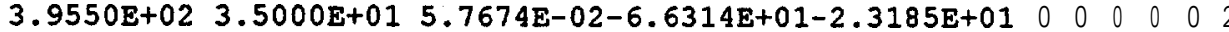

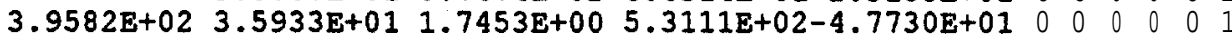

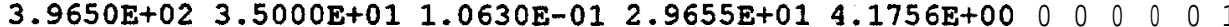

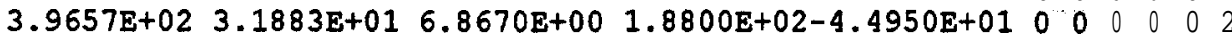

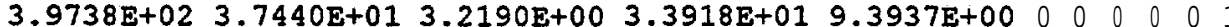

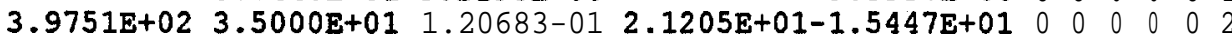

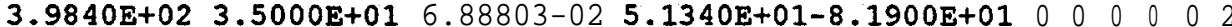

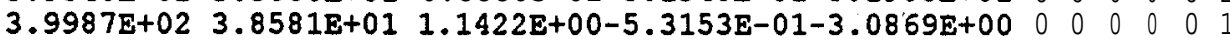

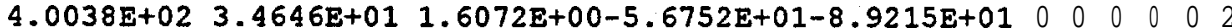

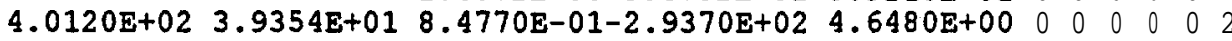
$4.0155 \mathrm{E}+02 \quad 3.3024 \mathrm{E}+01 \quad 1.0822 \mathrm{E}+00-8.9714 \mathrm{E}-01-1.4994 \mathrm{E}+00$ $4.0235 \mathrm{E}+02 \quad 3.2651 \mathrm{E}+012.6088 \mathrm{E}+00 \quad 1.5317 \mathrm{E}+02-2.9358 \mathrm{E}+02$ $4.0290 \mathrm{E}+023.4561 \mathrm{E}+018.69243-011.6338 \mathrm{E}+02-6.3174 \mathrm{E}+02$ $4.0320 \mathrm{E}+02 \quad 3.5000 \mathrm{E}+013.33603-019.7200 \mathrm{E}+01-8.2230 \mathrm{E}+01$ 4.0420E+02 3.5000E+01 2.07903-01 1.0500E+02 2.6580E+01 $4.0465 \mathrm{E}+02 \quad 3.3121 \mathrm{E}+014.1881 \mathrm{E}+00-6.3471 \mathrm{E}+01-1.4088 \mathrm{E}+02$ $4.0505 \mathrm{E}+02 \quad 3.0377 \mathrm{E}+012.4367 \mathrm{E}+001.5677 \mathrm{E}+02-9.2204 \mathrm{E}+01$ $4.0578 \mathrm{E}+02 \quad 2.9262 \mathrm{E}+01 \quad 3.3800 \mathrm{E}+001.0237 \mathrm{E}+02-2.5592 \mathrm{E}+02$ $4.0580 \mathrm{E}+02 \quad 3.5000 \mathrm{E}+01 \quad 1.0050 \mathrm{E}-01 \quad 9.1800 \mathrm{E}+01-1.6000 \mathrm{E}+02$ $4.0590 \mathrm{E}+02 \quad 3.1326 \mathrm{E}+01 \quad 1.9714 \mathrm{E}+00-4.8449 \mathrm{E}+02 \quad 5.6247 \mathrm{E}+01$ 4.0690E+02 3.5000E+01 $2.32803-01 \quad 6.9280 \mathrm{E}+01-6.9790 \mathrm{E}+01$ $4.0730 \mathrm{E}+02 \quad 3.3112 \mathrm{E}+018.06403-016.5680 \mathrm{E}+01-1.3790 \mathrm{E}+02$ $4.0748 \mathrm{E}+02 \quad 3.5000 \mathrm{E}+01 \quad 1.86773-02 \quad 6.9794 \mathrm{E}+01-4.0155 \mathrm{E}+01$ $4.0799 \mathrm{E}+02 \quad 2.9122 \mathrm{E}+012.3834 \mathrm{E}+00 \quad 3.6192 \mathrm{E}+02-1.5342 \mathrm{E}+02$ $4.0854 \mathrm{E}+02 \quad 3.2643 \mathrm{E}+01 \quad 4.4817 \mathrm{E}+00 \quad 1.7117 \mathrm{E}+02-8.5868 \mathrm{E}+01$ $4.0903 \mathrm{E}+02 \quad 3.5000 \mathrm{E}+018.0201 \mathrm{E}-02-5.5304 \mathrm{E}+019.8720 \mathrm{E}+01$ $4.1000 \mathrm{E}+02 \quad 3.5000 \mathrm{E}+01 \quad 1.79903-011.9710 \mathrm{E}+02-8.5540 \mathrm{E}+01$ $4.1056 \mathrm{E}+02 \quad 3.6251 \mathrm{E}+01 \quad 1.8105 \mathrm{E}+00 \quad 4.8993 \mathrm{E}+01-6.3977 \mathrm{E}+01$ $4.1079 \mathrm{E}+02 \quad 3.5000 \mathrm{E}+01 \quad 4.2202 \mathrm{E}-01 \quad 4.2595 \mathrm{E}+01-3.3733 \mathrm{E}+01$ $4.1100 \mathrm{E}+02 \quad 3.5000 \mathrm{E}+01 \quad 2.7948 \mathrm{E}-01 \quad 7.8936 \mathrm{E}+01-1.3458 \mathrm{E}+02$ $4.1217 \mathrm{E}+02 \quad 3.5000 \mathrm{E}+013.50413-014.1131 \mathrm{E}+01-6.1742 \mathrm{E}+01$ $4.1325 \mathrm{E}+02 \quad 3.5000 \mathrm{E}+017.9878 \mathrm{E}-02 \quad 8.9417 \mathrm{E}+01-8.6234 \mathrm{E}+00$ $4.1350 \mathrm{E}+02$ 3.5000E+01 8.53003-02 8.3000E+01-6.5170E+01 $4.1408 \mathrm{E}+02 \quad 3.6567 \mathrm{E}+012.9129 \mathrm{E}+00-4.9634 \mathrm{E}+01-3.1724 \mathrm{E}+02$ 4.1440E+02 3.5000E+01 $1.48303-01 \quad 7.9420 \mathrm{E}+01-5.8040 \mathrm{E}+01$ 4.1500E+02 3.4001E+01 3.6253E+00-4.4058E+01-1.0193E+00 $4.1557 \mathrm{E}+02 \quad 3.2710 \mathrm{E}+01 \quad 1.8214 \mathrm{E}+00 \quad 2.4857 \mathrm{E}+00 \quad 4.2512 \mathrm{E}+02$ $4.1560 \mathrm{E}+02 \quad 3.5000 \mathrm{E}+01 \quad 6.28503-012.1890 \mathrm{E}+02-7.3840 \mathrm{E}+01$ $4.1577 \mathrm{E}+02 \quad 2.8768 \mathrm{E}+01 \quad 1.4216 \mathrm{E}+00-1.1670 \mathrm{E}+02 \quad 8.8590 \mathrm{E}+01$ $4.1680 \mathrm{E}+02 \quad 3.5000 \mathrm{E}+011.6750 \mathrm{E}-01-5.9410 \mathrm{E}+02-1.3680 \mathrm{E}+02$ $4.1798 \mathrm{E}+02 \quad 3.5000 \mathrm{E}+012.9985 \mathrm{E}-01-9.4669 \mathrm{E}+01 \quad 6.9408 \mathrm{E}+00$ $4.1822 \mathrm{E}+02 \quad 3.1199 \mathrm{E}+01 \quad 4.5857 \mathrm{E}+00 \quad 6.0868 \mathrm{E}+01-2.8478 \mathrm{E}+01$ $4.1826 \mathrm{E}+022.5818 \mathrm{E}+01 \quad 5.5407 \mathrm{E}+001.5139 \mathrm{E}+01 \quad 5.9325 \mathrm{E}+01$ $4.1986 \mathrm{E}+02 \quad 4.9695 \mathrm{E}+01 \quad 5.9809 \mathrm{E}+00 \quad 1.6715 \mathrm{E}+02-6.8884 \mathrm{E}+01$ $\begin{array}{llllll}4.1989 \mathrm{E}+02 & 3.7883 \mathrm{E}+01 & 1.9114 \mathrm{E}+00 & 7.5112 \mathrm{E}+02 & 1.0683 \mathrm{E}+01\end{array}$ $4.2044 \mathrm{E}+02 \quad 3.5000 \mathrm{E}+018.00603-02 \quad 6.7759 \mathrm{E}+016.2773 \mathrm{E}+01$ $4.2050 \mathrm{E}+02 \quad 3.5000 \mathrm{E}+01$ 1.9890E-02-4.9911 $\mathrm{E}+007.3899 \mathrm{E}+00$ $4.2161 \mathrm{E}+02 \quad 3.5000 \mathrm{E}+01 \quad 1.89513-013.4541 \mathrm{E}+02-5.5793 \mathrm{E}+02$ $4.2163 \mathrm{E}+02 \quad 3.5000 \mathrm{E}+01 \quad 4.5562 \mathrm{E}-01 \quad 1.3597 \mathrm{E}+02-2.3242 \mathrm{E}+02$ 4.2254E+02 3.5000E+01 3.51673-01 1.4219E+02-1.2192E+02 $4.2276 \mathrm{E}+02 \quad 3.5000 \mathrm{E}+01 \quad 5.0019 \mathrm{E}-02-2.1895 \mathrm{E}+00 \quad 1.8382 \mathrm{E}+02$ $4.2305 \mathrm{E}+02 \quad 3.5150 \mathrm{E}+012.8856 \mathrm{E}+00 \quad 1.5802 \mathrm{E}+02-6.9033 \mathrm{E}+02$ $4.2338 \mathrm{E}+022.7049 \mathrm{E}+019.0151 \mathrm{E}+00-2.8611 \mathrm{E}-01-5.8010 \mathrm{E}+02$ $4.2392 \mathrm{E}+02 \quad 3.2620 \mathrm{E}+012.1349 \mathrm{E}+00 \quad 2.0968 \mathrm{E}+02-3.8955 \mathrm{E}+02$ $4.2400 \mathrm{E}+02 \quad 3.5000 \mathrm{E}+011.3679 \mathrm{E}-01-2.5340 \mathrm{E}+02-6.1210 \mathrm{E}+01$ $4.2429 \mathrm{E}+02 \quad 3.1513 \mathrm{E}+011.7642 \mathrm{E}+00 \quad 5.4116 \mathrm{E}+01-5.4686 \mathrm{E}+01$ $4.2451 \mathrm{E}+02 \quad 3.5000 \mathrm{E}+01 \quad 1.0490 \mathrm{E}-01 \quad 9.5520 \mathrm{E}+01-4.1397 \mathrm{E}+01$ $4.2522 \mathrm{E}+023.6188 \mathrm{E}+017.9475 \mathrm{E}+00-3.8494 \mathrm{E}+01-4.0374 \mathrm{E}+00$ $4.2550 \mathrm{E}+02 \quad 3.5000 \mathrm{E}+011-58433-011.9477 \mathrm{E}+01-6.1617 \mathrm{E}+01$ $4.2609 \mathrm{E}+02 \quad 3.4406 \mathrm{E}+01 \quad 6.3387 \mathrm{E}+00 \quad 5.4663 \mathrm{E}+02-7.8144 \mathrm{E}+01$ $4.2650 \mathrm{E}+02 \quad 3.5000 \mathrm{E}+01 \quad 1.0559 \mathrm{E}-01-9.0978 \mathrm{E}+02-1.6956 \mathrm{E}+01$ $4.2747 \mathrm{E}+022.9386 \mathrm{E}+01 \quad 4.4097 \mathrm{E}+00-6.3884 \mathrm{E}+01-8.2127 \mathrm{E}+01$ $4.2878 \mathrm{E}+02 \quad 3.7448 \mathrm{E}+013.1712 \mathrm{E}+00 \quad 1.1050 \mathrm{E}+02-2.3246 \mathrm{E}+01$ $4.2943 \mathrm{E}+023.6380 \mathrm{E}+01 \quad 4.1479 \mathrm{E}+002.0722 \mathrm{E}+01-2.2892 \mathrm{E}+01$ $4.3040 \mathrm{E}+023.3802 \mathrm{E}+016.3712 \mathrm{E}+002.0359 \mathrm{E}+02-4.1136 \mathrm{E}+02$ 
$\begin{array}{lllllllllll}4.3121 E+02 & 3.1377 \mathrm{E}+01 & 4.3093 \mathrm{E}+00 & 1.5292 \mathrm{E}+02-1.5796 \mathrm{E}+02 & 0 & 0 & 0 & 0 & 6 & 1\end{array}$ $4.3150 \mathrm{E}+02 \quad 3.5000 \mathrm{E}+01 \quad 1.0167 \mathrm{E}-01 \quad 1.1091 \mathrm{E}+02 \quad 3.4043 \mathrm{E}+0200000012$

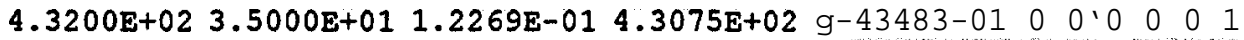

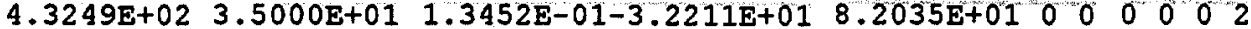

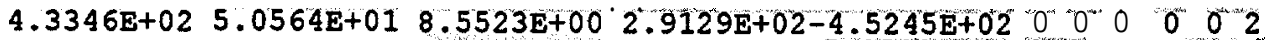

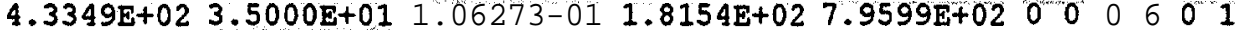
$\begin{array}{lllllllllllllll}4.3390 \mathrm{E}+02 & 2.8140 \mathrm{E}+01 & 7.7487 \mathrm{E}+00 & 1.0809 \mathrm{E}+02 & 6.5897 \mathrm{E}+01 & 0 & 0 & 0 & 0 & 2\end{array}$ $4.3470 \mathrm{E}+02 \quad 3.5000 \mathrm{E}+01 \quad 9.4320 \mathrm{E}-02-1.5634 \mathrm{E}+02-9.2748 \mathrm{E}+02 \quad 0 \quad 0 \quad 0 \quad 002$ $\begin{array}{lllllllllllllllllll}4.3488 \mathrm{E}+02 & 2.9079 \mathrm{E}+01 & 6.9459 \mathrm{E}+00 & 6.9176 \mathrm{E}+01-5.8011 \mathrm{E}+01 & 0 & 0 & 0 & 0 & 0 & 1\end{array}$ $4.3528 \mathrm{E}+023.5000 \mathrm{E}+01 \quad 6.3256 \mathrm{E}-02 \quad 3.1879 \mathrm{E}+01-2.0551 \mathrm{E}+00000001002$ $\begin{array}{llllllllll}4.3629 \mathrm{E}+02 & 3.5000 \mathrm{E}+01 & 1-34323-01 & 9.9222 \mathrm{E}-01-2.9760 \mathrm{E}+02 & 0 & 0 & 0 & 0 & 0 & -2\end{array}$ $\begin{array}{llllllllllll}4.3638 \mathrm{E}+02 & 3.5000 \mathrm{E}+01 & 2.3756 \mathrm{E}-01 & 9.8640 \mathrm{E}+01-5.0604 \mathrm{E}+01 & 6 & 0 & 0 & 6 & 6\end{array}$

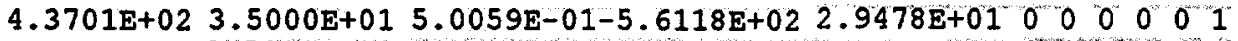

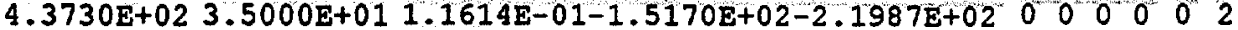
$4.3803 \mathrm{E}+02 \quad 3.5000 \mathrm{E}+01 \quad 3.8591 \mathrm{E}-01 \quad 2.1213 \mathrm{E}+02-3.9461 \mathrm{E}+01 \quad 0 \quad 0 \quad 0 \quad 0 \quad 01$ $4.3880 \mathrm{E}+02 \quad 4.7884 \mathrm{E}+01 \quad 3.8199 \mathrm{E}+00 \quad 2.9697 \mathrm{E}+02 \quad 3.5883 \mathrm{E}+02 \quad 0 \quad 0 \quad 00000$

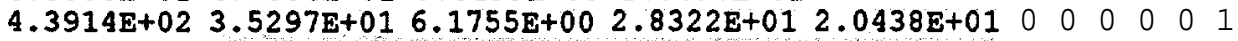
$4.3976 \mathrm{E}+022.8547 \mathrm{E}+01 \quad 2.0816 \mathrm{E}+00-4.4019 \mathrm{E}+01-4.8236 \mathrm{E}+0100000$ $4.4039 \mathrm{E}+02 \quad 3.5164 \mathrm{E}+01 \quad 1.0573 \mathrm{E}+013.6323 \mathrm{E}+01-1.1356 \mathrm{E}+02 \quad 0 \quad 000002$

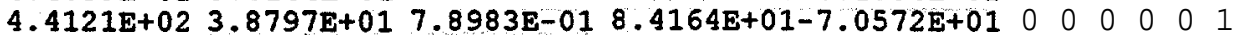

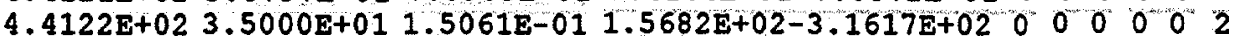
$4.4216 \mathrm{E}+02 \quad 3.0522 \mathrm{E}+01 \quad 9.9543 \mathrm{E}+00 \quad 2.4062 \mathrm{E}+02 \quad 2.2866 \mathrm{E}+02 \quad 0000002$ $4.4320 \mathrm{E}+023.5000 \mathrm{E}+01$ 7.0108E-02-9.7530E+01-6.7823E+01 0 O 000 O 02 $\begin{array}{llllllllllllll}4.4352 \mathrm{E}+02 & 3.5000 \mathrm{E}+01 & 2.1900 \mathrm{E}-01-9.8614 \mathrm{E}+01 & 6.3785 \mathrm{E}+00 & 0 & 0 & 0 & 0 & 0 & 1\end{array}$

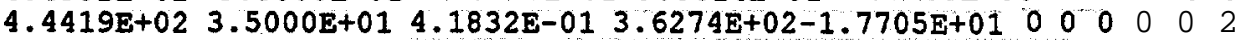
$4.4439 \mathrm{E}+02 \quad 3.5000 \mathrm{E}+01 \quad 6.7615 \mathrm{E}-01 \quad 1.6226 \mathrm{E}+02 \quad 3.2231 \mathrm{E}+010000001$ $4.4490 \mathrm{E}+02 \quad 3.5000 \mathrm{E}+01 \quad 6.3292 \mathrm{E}-02-6.4048 \mathrm{E}+02-3.3639 \mathrm{E}+02$ o 0 o 00 $4.4538 \mathrm{E}+02 \quad 3.8457 \mathrm{E}+01 \quad 9.8768 \mathrm{E}-01 \quad 6.4760 \mathrm{E}+01-6.0258 \mathrm{E}+01 \quad 0 \quad 000001$ $4.4574 \mathrm{E}+023.2750 \mathrm{E}+017.0600 \mathrm{E}-013.1967 \mathrm{E}+013.8110 \mathrm{E}+01 \quad 0000002$ $4.4700 \mathrm{E}+023.5000 \mathrm{E}+01 \quad 8.5170 \mathrm{E}-02 \quad 1.9140 \mathrm{E}+01 \quad 7.9860 \mathrm{E}+01$ o 000002

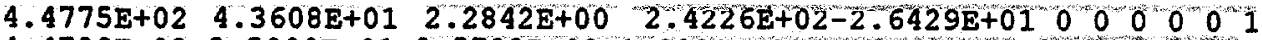
$4.4789 \mathrm{E}+02 \quad 3.5000 \mathrm{E}+01 \quad 8.5782 \mathrm{E}-02-1.0281 \mathrm{E}+02-2.8569 \mathrm{E}+02 \quad 0 \quad 0 \quad 0002$ $\begin{array}{llllllllllll}4.4868 \mathrm{E}+02 & 3.0670 \mathrm{E}+01 & 4.9374 \mathrm{E}+00 & 4.0518 \mathrm{E}+01 & 1.3265 \mathrm{E}+01 & 0 & 0 & 0 & 0 & 0 & 2\end{array}$

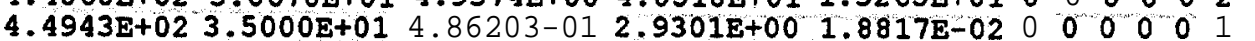
$4.4978 \mathrm{E}+025.0432 \mathrm{E}+015.9829 \mathrm{E}+002.9168 \mathrm{E}+02-4.0414 \mathrm{E}+010000002$ $4.5071 \mathrm{E}+023.9793 \mathrm{E}+01 \quad 2.8644 \mathrm{E}+00 \quad 5.8815 \mathrm{E}+01-1.2190 \mathrm{E}+0200000$ $4.5079 \mathrm{E}+023.5000 \mathrm{E}+011.6102 \mathrm{E}-016.4570 \mathrm{E}+024.1525 \mathrm{E}+01$ o $0^{4} 00^{\circ} 2$ $4.5160 \mathrm{E}+023.5000 \mathrm{E}+01 \quad 1.3220 \mathrm{E}-01 \quad 1.0143 \mathrm{E}+02-8.6795 \mathrm{E}+01000002$ $\begin{array}{lllllllllllllllllllllllllll}4.5200 \mathrm{E}+02 & 3.5000 \mathrm{E}+01 & 8.7779 \mathrm{E}-02-1.1685 \mathrm{E}+00 & 9.4765 \mathrm{E}+01 & 0 & 0 & 0 & 0 & 1\end{array}$ $4.5221 \mathrm{E}+023.5000 \mathrm{E}+012.8406 \mathrm{E}-01 \quad 3.6300 \mathrm{E}+02 \quad 1.2306 \mathrm{E}+020000012$ $4.5297 \mathrm{E}+023.8192 \mathrm{E}+019.8996 \mathrm{E}-019.2266 \mathrm{E}+01-4.2621 \mathrm{E}+020000001$ $4.5362 \mathrm{E}+02 \quad 3.6540 \mathrm{E}+01 \quad 5.6572 \mathrm{E}+00-1.7099 \mathrm{E}+01 \quad 5.6654 \mathrm{E}+01 \quad 0 \quad 000002$ $4.5413 \mathrm{E}+023.3580 \mathrm{E}+012.0641 \mathrm{E}+003.2270 \mathrm{E}+01-7.5962 \mathrm{E}+010000010$ $4.5430 \mathrm{E}+02 \quad 3.5000 \mathrm{E}+01$ 1.4146E-01-2.0758 $1027.7632 \mathrm{E}+0100001$ $4.5463 \mathrm{E}+023.5000 \mathrm{E}+01 \quad 1.5678 \mathrm{E}-01-6.5764 \mathrm{E}+01 \quad 4.6610 \mathrm{E}+02 \quad 0000002$ $4.5560 \mathrm{E}+023.5000 \mathrm{E}+018.3011 \mathrm{E}-0213136 \mathrm{E}+025.0697 \mathrm{E}+02$ o 00002

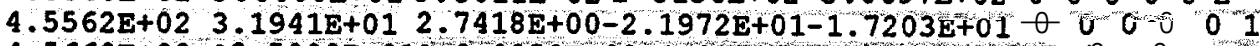

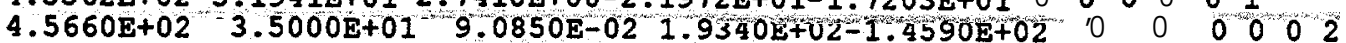

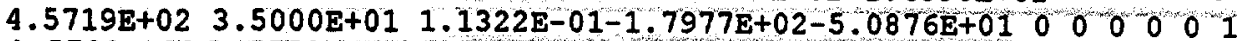
$4.5790 \mathrm{E}+023.5753 \mathrm{E}+01$ 1.1247E+00 1.1641E+02-3.6672 $\mathrm{E}+02000000$ $\begin{array}{llllllllllllll}4.5859 \mathrm{E}+02 & 3.5000 \mathrm{E}+01 & 1.3598 \mathrm{E}-01 & 3.1071 \mathrm{E}+00 & 8.1400 \mathrm{E}+00 & 0 & 0 & 0 & 0 & 0 & 2\end{array}$ $\begin{array}{lllllllllll}4.5876 \mathrm{E}+02 & 3.8815 \mathrm{E}+01 & 5.8457 \mathrm{E}+00 & 3.8170 \mathrm{E}+02-1.3436 \mathrm{E}-01 & 0 & 0 & 0 & 0 & 0 & 1 \\ 4.5957 \mathrm{E}+02 & 2.9828 \mathrm{E}+01 & 3.5796 \mathrm{E}+00 & 4.6494 \mathrm{E}+01 & 7.3839 \mathrm{E}+01 & 0 & 0 & 0 & 0 & 0 & 2\end{array}$ 4. $6009 \mathrm{E}+023.5000 \mathrm{E}+011.1512 \mathrm{E}-019.6528 \mathrm{E}+027.0123 \mathrm{E}+02$ on o o 0 o 1

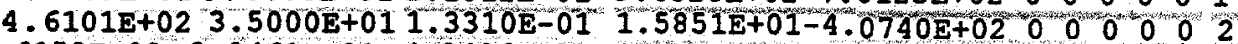
$\begin{array}{lllllllllllll}4.6152 \mathrm{E}+02 & 3.8461 \mathrm{E}+01 & 4.3496 \mathrm{E}+00 & 9.0827 \mathrm{E}+02 & 2.5482 \mathrm{E}+02 & 0 & 0 & 0 & 0 & 1\end{array}$ $4.6184 \mathrm{E}+023.7318 \mathrm{E}+01 \quad 1.1469 \mathrm{E}+019.1250 \mathrm{E}+00-2.0259 \mathrm{E}+01$ o $0 \mathrm{~B}$ $4.6284 \mathrm{E}+025.3347 \mathrm{E}+012.4486 \mathrm{E}+00-5.3240 \mathrm{E}+014.5465 \mathrm{E}+02000002$ $4.6334 \mathrm{E}+023.5000 \mathrm{E}+011.9694 \mathrm{E}-01-2.0185 \mathrm{E}+01-1.8074 \mathrm{E}+01$ o 0 o 02 4. $6380 \mathrm{E}+023.5849 \mathrm{E}+01$ 1.7642 $\mathrm{E}+01-2.8733 \mathrm{E}+00-2.8198 \mathrm{E}+02$ o 0 o 0.01

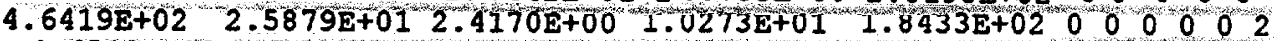
$4.6513 \mathrm{E}+023.5000 \mathrm{E}+019.6660 \mathrm{E}-02-4.7735 \mathrm{E}+02-6.2470 \mathrm{E}+01$ o 00002 $4.6562 \mathrm{E}+023.5000 \mathrm{E}+01 \quad 9.1594 \mathrm{E}-02-1.0426 \mathrm{E}+02-2.8172 \mathrm{E}+02 \quad 0 \quad 0000001$ $4.6610 \mathrm{E}+023.5000 \mathrm{E}+01 \quad 7.7436 \mathrm{E}-02 \quad 2.0539 \mathrm{E}+02 \quad 4.0808 \mathrm{~B}+0100000.2$

$4.6645 \mathrm{E}+02 \quad 3.5193 \mathrm{E}+01 \quad 3.2211 \mathrm{E}+00 \quad 2.1248 \mathrm{E}+02-2.3505 \mathrm{E}+01 \quad 0 \quad 000001$ $4.6650 \mathrm{E}+023.5000 \mathrm{E}+012.4907 \mathrm{E}-01 \quad 6.4868 \mathrm{E}+01-1.0196 \mathrm{E}+02000001$ 
4.6714E+02 3.5911E+01 1.1596E+00-3.1957E+01-3.7807E+02 00000000 $4.6851 \mathrm{E}+023.8483 \mathrm{E}+012.6582 \mathrm{E}+00-2.5077 \mathrm{E}+021.0534 \mathrm{E}+02 \quad 0^{\prime} 00000012^{\prime}$

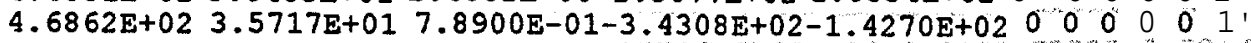

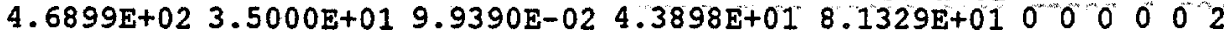
$\begin{array}{lllllllllll}4.6907 \mathrm{E}+02 & 3.3230 \mathrm{E}+01 & 4.8111 \mathrm{E}+00 & 5.4140 \mathrm{E}+01-4.7567 \mathrm{E}+02 & 0 & 0 & 0 & 0 & 0 & 1\end{array}$

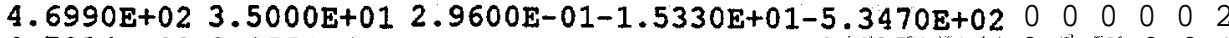
$4.7034 \mathrm{E}+02 \quad 3.2551 \mathrm{E}+01 \quad 1.2376 \mathrm{E}+00-3.2339 \mathrm{E}+01 \quad 1.1256 \mathrm{E}+02 \quad 0 \quad 000001$ $\begin{array}{lllllllllllll}4.7094 \mathrm{E}+02 & 3.5000 \mathrm{E}+01 & 1.4697 \mathrm{E}-01-4.8675 \mathrm{E}+01 & 2.3931 \mathrm{E}+02 & 0 & 0 & 0 & 0 & 0 & 2\end{array}$ $4.7179 \mathrm{E}+02 \quad 3.5000 \mathrm{E}+01 \quad 1.3400 \mathrm{E}-01-2.3258 \mathrm{E}+013.3017 \mathrm{E}+02 \quad 0100001$

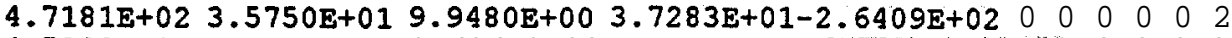

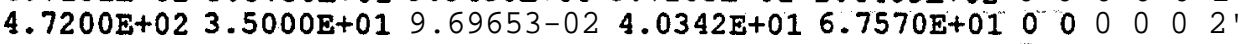

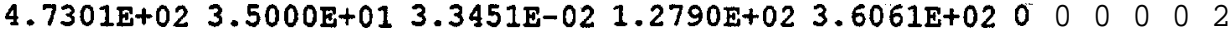
$4.7338 \mathrm{E}+02 \quad 3.0644 \mathrm{E}+01 \quad 1.8593 \mathrm{E}+00 \quad 2.1967 \mathrm{E}+01-7.3296 \mathrm{E}+0100000001$

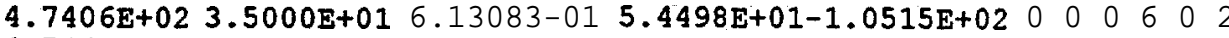
4.7461E+02 3.5000E+01 4.5266E-02 1.0465E+02-1.5777E+02 000 0'0 02

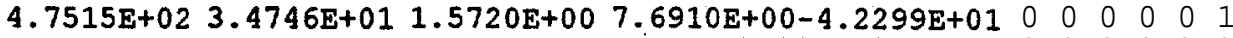

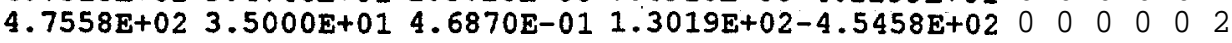

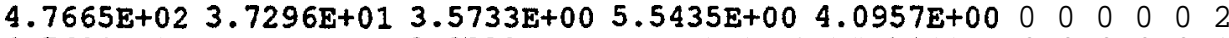

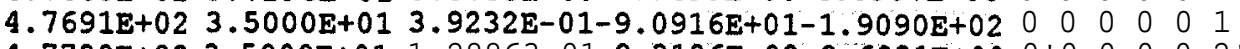

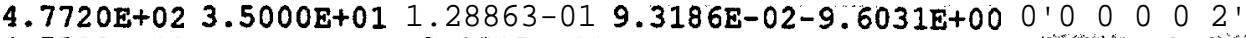
$\begin{array}{lllllllllllllll}4.7732 \mathrm{E}+02 & 3.6041 \mathrm{E}+01 & 3.8515 \mathrm{E}+00 & 1.7118 \mathrm{E}+02-2.9243 \mathrm{E}+01 & 0 & 0 & 0 & 6 & 0 & 1\end{array}$

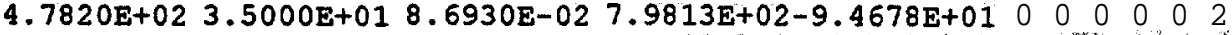
$\begin{array}{llllllllllllll}4.7900 \mathrm{E}+02 & 3.5000 \mathrm{E}+01 & \mathrm{E}-23653-02 & 2.1677 \mathrm{E}+02-2.3107 \mathrm{E}+01 & 0 & 0 & 0 & 0 & 0 & 1\end{array}$ $\begin{array}{lllllllllll}4.7924 \mathrm{E}+02 & 3.0126 \mathrm{E}+01 & 1.2688 \mathrm{E}+01 & 1.0028 \mathrm{E}+02 & 7.8848 \mathrm{E}+01 & 0 & 0 & 0 & 0 & 0 & 2\end{array}$

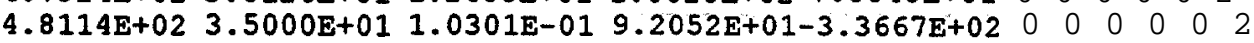

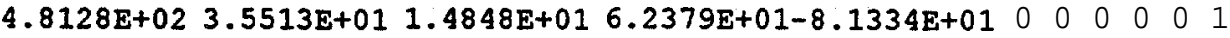

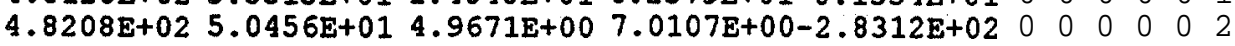

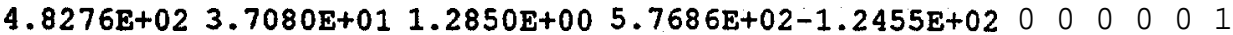

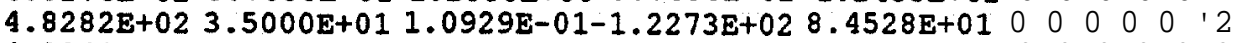

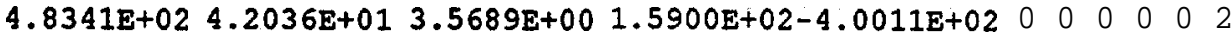

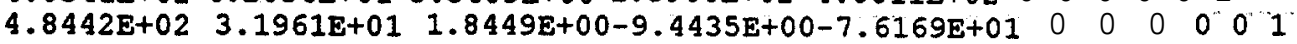
$4.8445 \mathrm{E}+023.5000 \mathrm{E}+011.0018 \mathrm{E}-01-5.1025 \mathrm{E}+022.1961 \mathrm{E}+020000000$

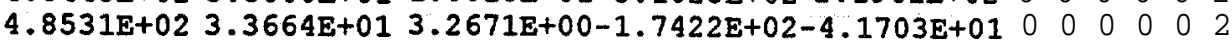

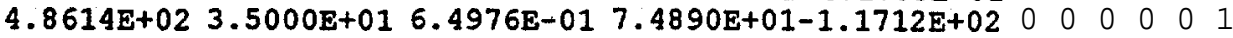

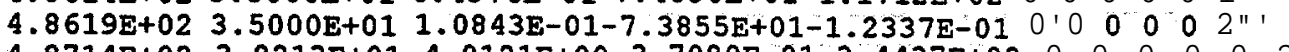
$\begin{array}{llllllllll}4.8714 E+02 & 3.8213 E+01 & 4.0121 E+00 & 3.7080 E-01-3.4437 E+02 & 0 & 0 & 0 & 0 & 0 & 2\end{array}$ $4.8789 \mathrm{E}+02 \quad 3.5000 \mathrm{E}+01 \quad 1-99693-01 \quad 1.3313 \mathrm{E}+02-2.6826 \mathrm{E}+02 \quad 0000001$

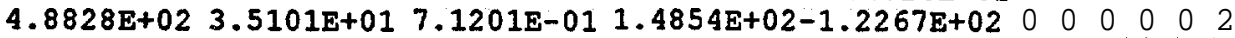
$\begin{array}{lllllllllll}4.8891 \mathrm{E}+02 & 3.5000 \mathrm{E}+01 & 2.4767 \mathrm{E}-01-2.2958 \mathrm{E}+01 & 1.4901 \mathrm{E}+01 & 0 & 0 & 0 & 0 & 0 & 0 & 0\end{array}$

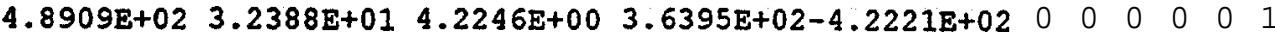

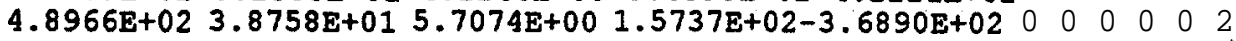

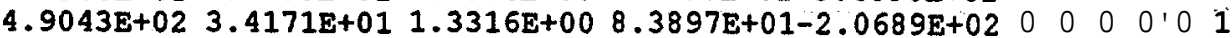

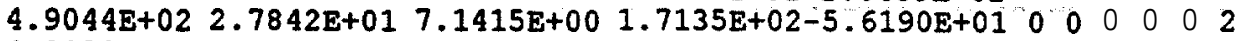

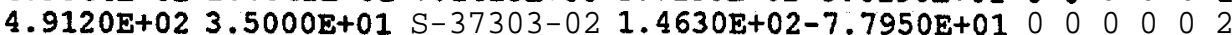

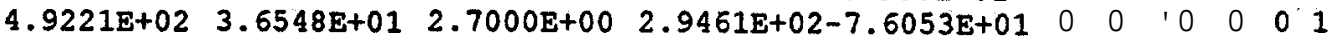

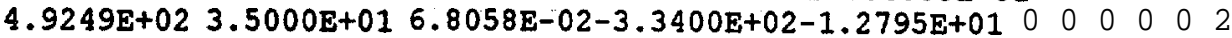
$4.9307 \mathrm{E}+02 \quad 4.0053 \mathrm{E}+01 \quad 3.9516 \mathrm{E}+00 \quad 2.3767 \mathrm{E}+01-9.7244 \mathrm{E}+00000000001$ $4.9396 \mathrm{E}+02 \quad 3.5164 \mathrm{E}+01$ 7.2048E-01-7.1355E+02-1.0812E+02 00000000

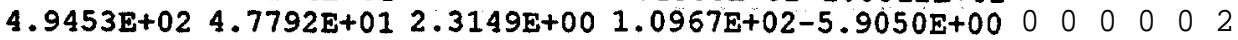
$4.9513 \mathrm{E}+02 \quad 3.9517 \mathrm{E}+01 \quad 1.3364 \mathrm{E}+00 \quad 1.4465 \mathrm{E}+02-5.0210 \mathrm{E}+0000000002$

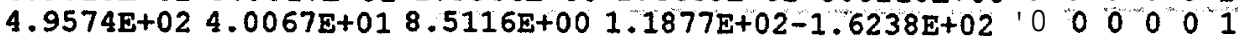

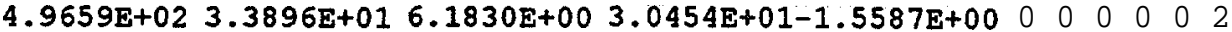

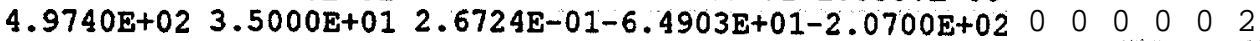

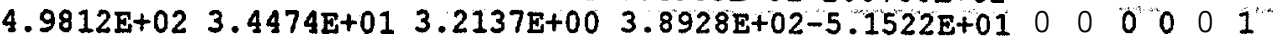
$4.9840 \mathrm{E}+02 \quad 3.5000 \mathrm{E}+01 \quad 1.20393-01 \quad 2.2416 \mathrm{E}+02-1.7506 \mathrm{E}+02 \quad 0000002$

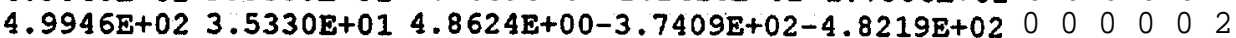
$4.9950 \mathrm{E}+02 \quad 3.4831 \mathrm{E}+013.8233 \mathrm{E}+00 \quad 7.3265 \mathrm{E}+02-9.7398 \mathrm{E}+01 \quad 0-0 \quad 0.001$

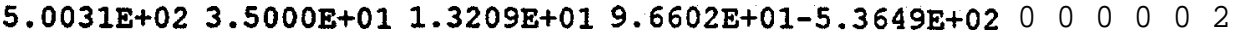
$5.0150 \mathrm{E}+02 \quad 3.5000 \mathrm{E}+015.9938 \mathrm{E}+00 \quad 9.3404 \mathrm{E}+02-7.5243 \mathrm{E}+0100000001$ $5.5000 \mathrm{E}+02 \quad 3.5000 \mathrm{E}+01 \quad 1.1304 \mathrm{E}+03 \quad 4.9670 \mathrm{E}+02-1.7531 \mathrm{E}+02 \quad 0 \quad 0 \quad 0.01^{\prime} 1$ 
ORNL/TM-13092

\title{
I N T E R N A L DISTRIBUTION
}

\author{
1. 0. Bouland \\ 2. H. Derrien \\ 3. F. Difillipo \\ 4. R. H. Fowler \\ 5. N. M. Greene \\ 6. J. A. Harvey \\ 7. D. M. Hetrick \\ 8. P. E. Koehler \\ 9. M. A. Kuliasha \\ 10. D. C. Larson \\ 11-15. N. M. Larson \\ 16-20. L. C. Leal \\ 21. L. F. Norris \\ 22. C. V. Parks \\ 23. S. Raman
}

\section{EXTERNAL DISTRIBUTION}

39; R. Block, Rensselaer Polytechnic Institute, Troy, NY 12 180-3590

40. J. Burke, Rensselaer Polytechnic Institute, Troy, NY 12 180-3 590

41. D. Cabrilla, U.S. Department of Energy, EM-23, 1000 Independence Avenue, Washington DC 20585-0002

42. D. E. Carlson, Reactor and Plant System Branch, Division of System Research, Office of Nuclear Regulatory Research, U.S. Nuclear Regulatory Commission, MS T-10 G6, RM T-10, I7, Washington, DC 20555-0001

43. R C. Carlton, Box 407, Middle Tennesse State University, Murfreesboro, TN 37132

44. R. L. Dintaman, U.S. Department of Energy, DP-13, Washington, DC 20585

45. C. Dunford, Bldg 197D, National Nuclear Data Center, Brookhaven National Laboratory, Upton, NY 11973

46. J. R Felty, DynCorp, Advanced Technology Services, 4401 Ford Avenue, Suite 300, Alexandria, VA 22302-1432

47. P. Finck, DER/SPRC/LEPH, Batiment 230, Centre d'Etudes de CADARACHE, 13108 Saint Paul-lez-Durance, France

48. C. M. Frankle, Los Alamos National Laboratory, P.O. Box 1663, Los Alamos, NM 87544

49. F. Froener, Kernforschungszentrum Karlsruhe, Institut fuer Neutronenphysik und Reacktortechnik, Postfach 336 40, D-76021 Karlsruhe, Germany 
50. R. N. Hwang, Argonne National Laboratory, Reactor Analysis Division, Bldg 208, Argonne, IL 60439

5 1. Y. Kikuchi, Nuclear Data Center, Japan Atomic Energy Research Institute, Tokaimura, Naka-gun, Ibaraki-ken 3 19-11, Japan

52. C. Lubitz, Knolls Atomic Power Laboratory, P. 0. Box 1072, Schnectady, NY 12301

53. R E. MacFarlane, Los Alamos National Laboratory, T2, MS342, Los Alamos, NM $\begin{array}{lllll}8 & 7 & 5 & 4 & 5\end{array}$

54. B. Moretti, Rensselaer Polytechnic Institute, Troy, NY 12180-3590

55. C. Nordborg, OECD/NEA, Le Seine St-Germain 12, Boulevard Iles, 92130 Issy-lesMoulineaux, France

56-57. Office of Scientific and Technical Information, U.S. Department of Energy, P.O. Box 62, Oak Ridge, TN 37831

58. Office of the ORNL Site Manager, Department of Energy, Oak Ridge National Laboratory, P.O. Box 2008, Oak Ridge, TN 37831

59. M. Salvatores, DRN/P, Batiment 707, C. E. CADARACHE, 13108 Saint Paul-lezDurance, France

60. C. Werner, Rensselaer Polytechnic Institute, Troy, NY 12180-3590

61. Roger White, Lawrence Liver-more National Laboratory, P. 0. Box 8.08, Livermore, CA 94550

62. M. Williams, Nuclear Science Center, Louisiana State University; Baton Rouge, LA 70803

63. R. R. Winters, Denison University, Physics Department, Granville, Ohio 43023 